\title{
A FLIGHT SIMULATION STUDY OF THE SIMULTANEOUS NON-INTERFERING AIRCRAFT APPROACH
}

\author{
A Thesis \\ presented to \\ the Faculty of California Polytechnic State University, \\ San Luis Obispo
}

In Partial Fulfillment

of the Requirements for the Degree

Master of Science in Aerospace Engineering

by

Brian Reel

May 2009 
(C) 2009

Brian Hogan Reel

ALL RIGHTS RESERVED 


\section{COMMITTEE MEMBERSHIP}

TITLE:

A Flight Simulation Study of the Simultaneous Non-Interfering

Aircraft Approach

AUTHOR:

Brian Hogan Reel

DATE SUBMITTED: $\quad$ May 2009

COMMITEE CHAIR: $\quad$ Dr. Daniel Biezad, Cal Poly Aerospace Engineering

COMMITTEE MEMBER: Craig Hange, NASA AMES

COMMITEE MEMBER: $\quad$ Dr. Eric Mehiel, Cal Poly Aerospace Engineering

COMMITTEE MEMBER: Dr. Frank Owen, Cal Poly Mechanical Engineering

COMMITTEE MEMBER: $\quad$ Dr. Kurt Colvin, Cal Poly Industrial/Manufacturing Engineering 


\begin{abstract}
A Flight Simulation Study of the Simultaneous Non-Interfering Aircraft Approach Brian Hogan Reel
\end{abstract}

Using a new implementation of a NASA flight simulation of the Quiet Short-Haul Research Aircraft, autopilots were designed to be capable of flying both straight in (ILS) approaches, and circling (SNI) approaches. A standard glideslope coupler was sufficient for most conditions, but a standard Proportional-Integral-Derivative (PID) based localizer tracker was not sufficient for maintaining a lateral track on the SNI course. To track the SNI course, a feed-forward system, using GPS steering provided much better results.

NASA and the FAA embrace the concept of a Simultaneous, Non-Interfering (SNI) approach as a way to increase airport throughput while reducing the noise footprints of aircraft on approach. The NASA concept for the SNI approach for Short Takeoff and Landing (STOL) aircraft involves a straight in segment flown above the flight path of a normal approach, followed by a spiraling descent to the runway. As this is a procedure that would be utilized by regional airliners, it is assumed that it would be conducted under Instrument Flight Rules (IFR).

GPS or INS guidance would be required to fly this approach, and it is likely that it would be necessary to fly the approach with a coupled autopilot: a stabilized, curving, instrument approach to decision altitude would be exceedingly difficult to fly. The autopilots in many current commuter and general aviation aircraft, however, were designed before the event of GPS, and do not have provisions for tracking curved paths. This study identifies problem areas in implementing the SNI circling approach on aircraft and avionics as they stand today and also gives examples of what can be done for the SNI approach to be successful. 


\section{ACKNOWLEDGMENTS}

I'd foremost like to thank my mother and father for their unwavering support. Without them I certainly would not be where I am today. My friends and general family deserve thanks as well, for always being here for me and for keeping me diligent. I greatly appreciate the work of Cassy Anthony and Natalia Sanchez, digitizing the figures from the QSRA Math Model, which became the foundation of my work. I am grateful for the help Grey Lake provided with editing this final report. I'd like to thank Dr. Daniel Biezad for his support as my thesis advisor. Thank you as well to Craig Hange of NASA and Professor David W. Hall for initiating this work through the Students With Attitude (SWAT) program at Cal Poly, San Luis Obispo. 


\section{TABLE OF CONTENTS}

LIST OF TABLES ..........................................................................................................viii

LIST OF FIGURES .........................................................................................................viii

LIST OF ACRONYMS ……………………………………………………………....

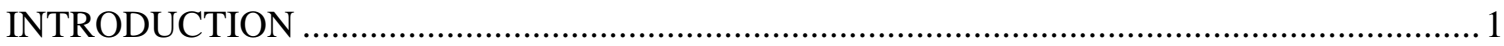

THE QUIET SHORT-HAUL RESEARCH AIRCRAFT ............................................................ 4

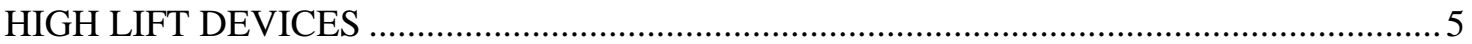

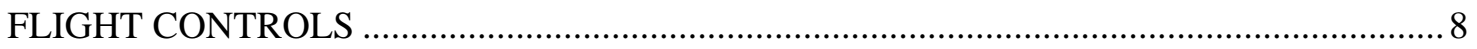

SIMLUTANEOUS NON-INTERFERING APPROACH ....................................................... 10

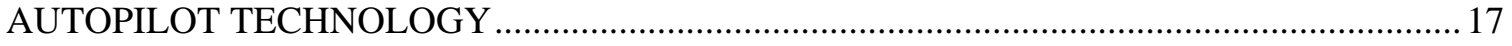

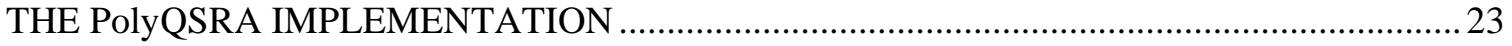

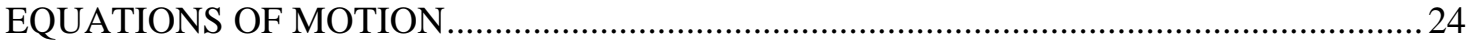

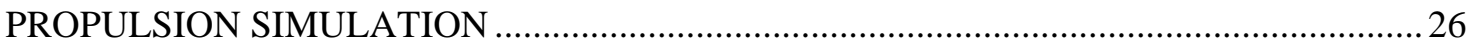

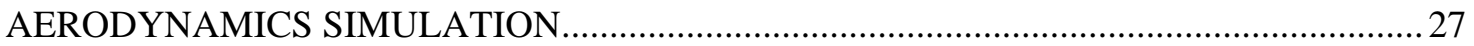

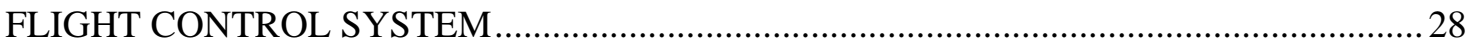

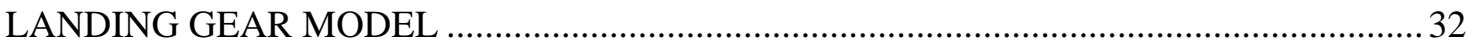

ATMOSPHERE MODEL ......................................................................................... 32

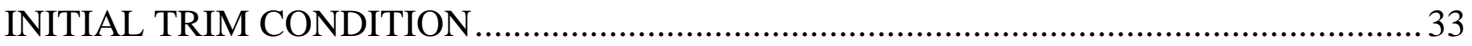

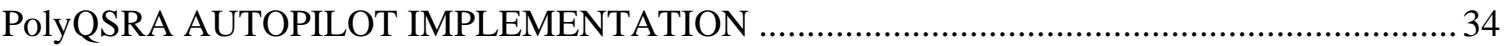

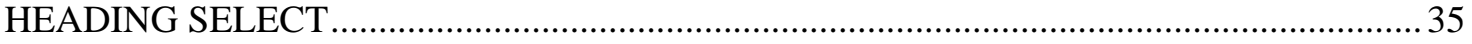

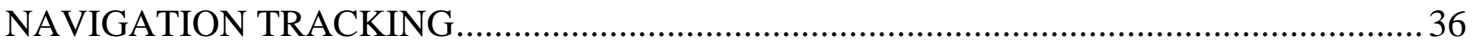

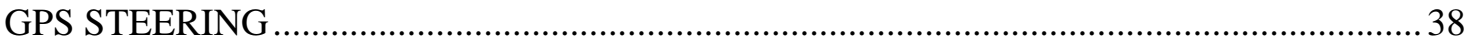

ALTITUDE HOLD/RATE COMMAND ………………………………………………..... 39

GLIDESLOPE COUPLER ………………………………………………………... 41

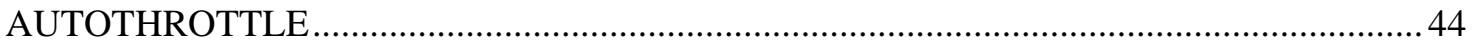

LOW SPEED AUTOPILOT PERFORMANCE ………………………………………..... 46

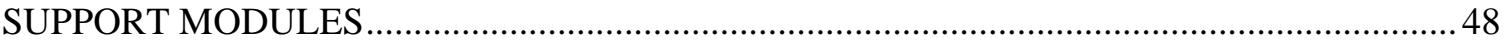

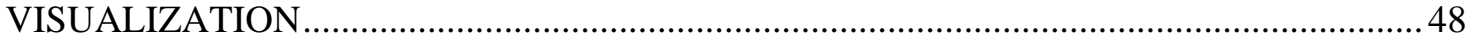

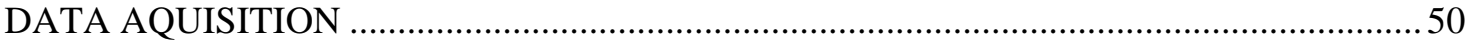

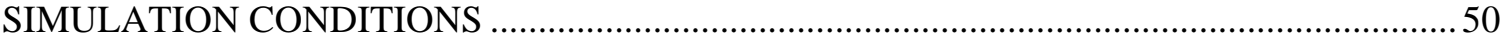

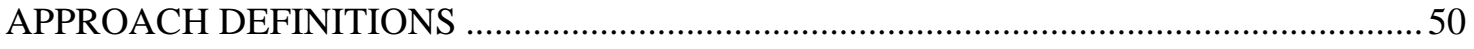

INTERCEPT CONDITIONS AND AIRCRAFT CONFIGURATION...................................... 51

TEST CONDITIONS ............................................................................................... 52 


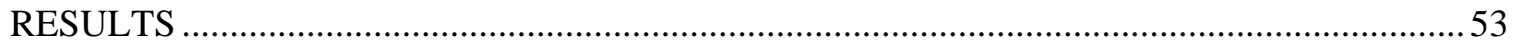

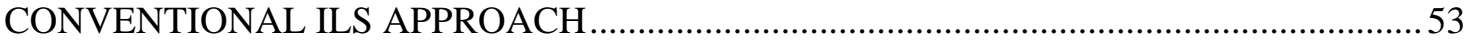

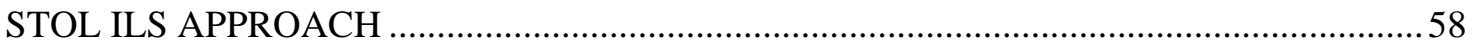

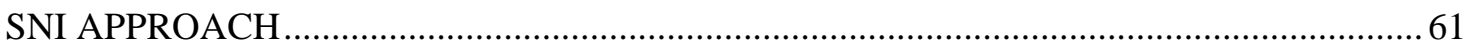

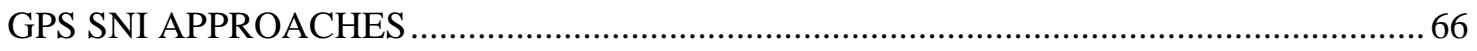

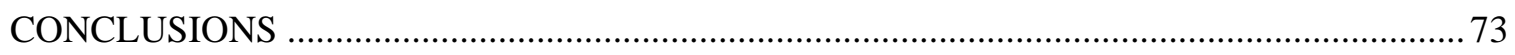

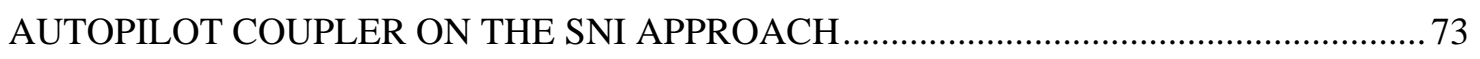

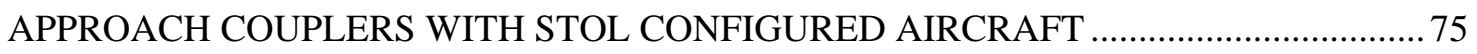

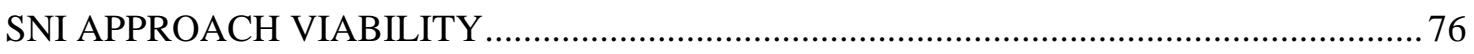

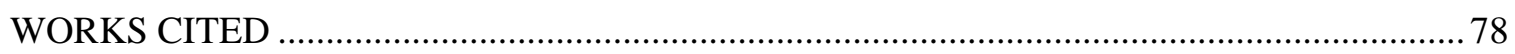

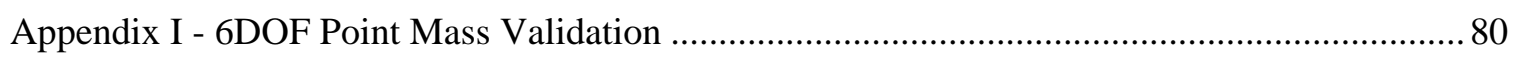

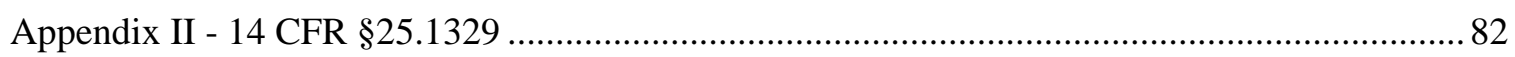

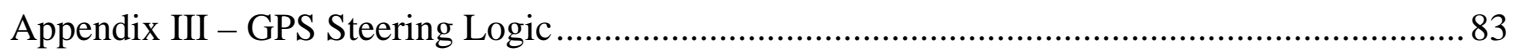




\section{LIST OF TABLES}

Table 1 - Test Conditions .52

\section{LIST OF FIGURES}

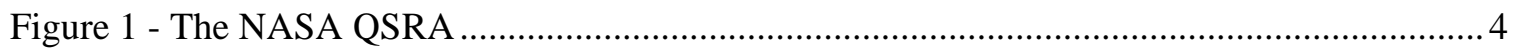

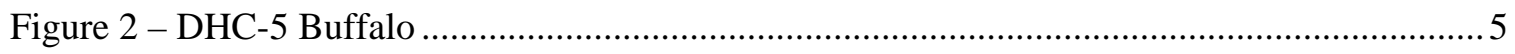

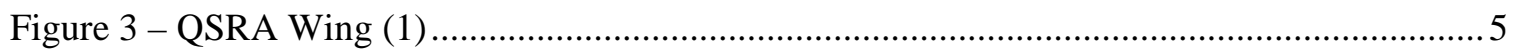

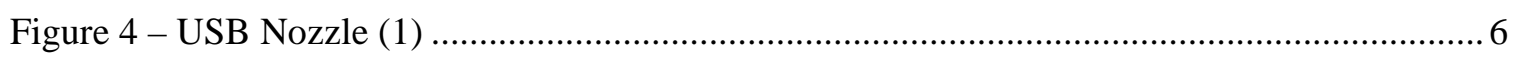

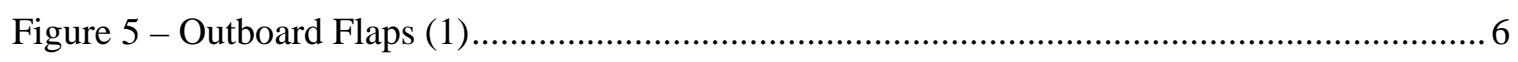

Figure 6 - BLC System and Cross-Ducting (4) …............................................................... 7

Figure 7 - Flight Control System Schematic ......................................................................... 8

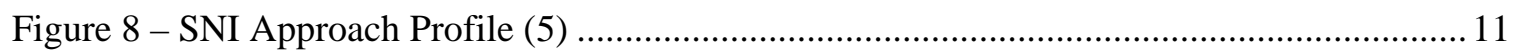

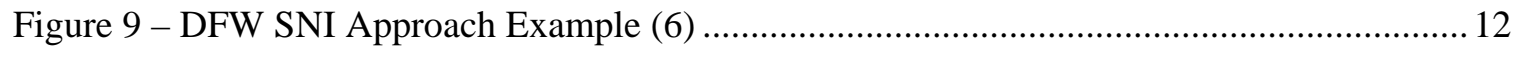

Figure 10 - Standard and SNI Approach Weighted Noise Contours (5) .................................... 13

Figure 11 - The Ouija Curve Approach Trajectory (5) …....................................................... 14

Figure 12 - Aggressive Continuous Descent SNI Approach (7) ............................................... 15

Figure 13 - C-17 SNI Approach Ground Tracks (5) ........................................................... 16

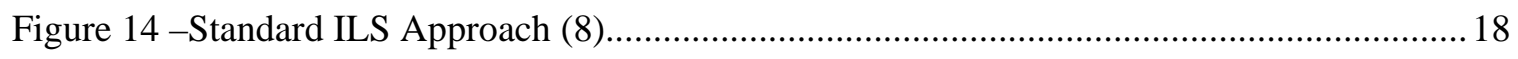

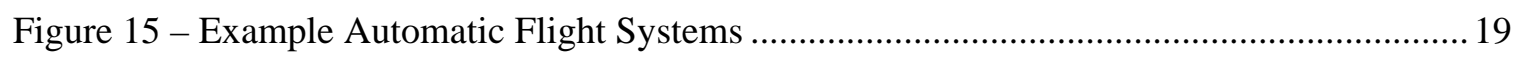

Figure 16 - Example Horizontal Situation Indicator (HSI) ..................................................... 20

Figure 17 - Example Math Model Parameter Figure (1) ............................................................. 24

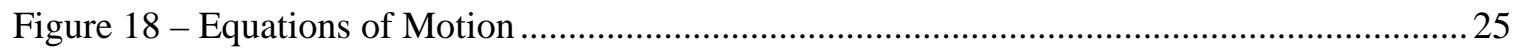

Figure 19 - Pitch Feel Computer Force Loader Gradients (1)..................................................29

Figure 20 - PolyQSRA Column Force Workaround ............................................................... 30 


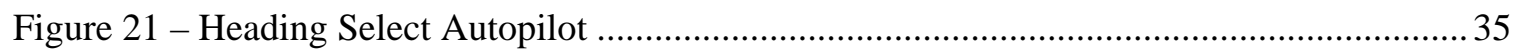

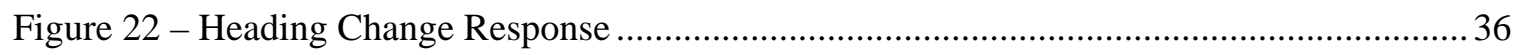

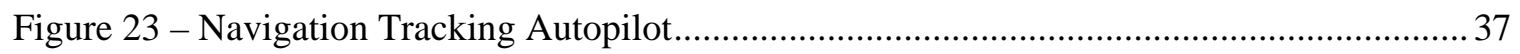

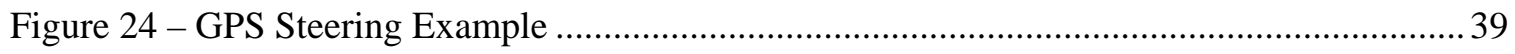

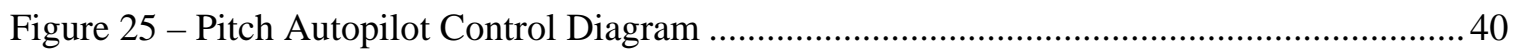

Figure 26 - Autopilot Altitude Change Response ............................................................... 41

Figure 27 - Altitude Hold in Moderate Turbulence .............................................................. 41

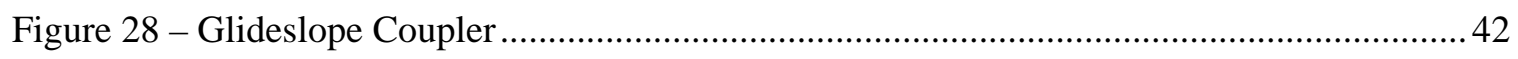

Figure 29 - Autothrottle Control Diagram ........................................................................ 44

Figure 30 - Autothrottle Speed Change Response .................................................................. 45

Figure 31 - Autothrottles Altitude Change Response ........................................................... 46

Figure 32 - Autothrottle Turbulence Response .................................................................... 46

Figure 33 - STOL Configuration Transitions .................................................................... 47

Figure 34 - Descent in the STOL Configuration ................................................................... 48

Figure 35 - FlightGear Visual Depiction............................................................................... 49

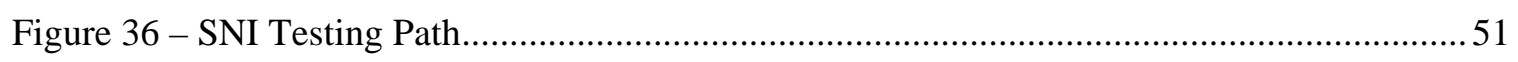

Figure 37 - CTOL ILS in Calm Conditions …................................................................... 54

Figure 38 - CTOL ILS Glidepath Performance..................................................................... 55

Figure 39 - CTOL ILS Turbulence Glidepath Performance ....................................................... 56

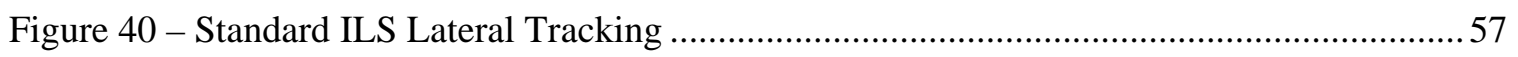

Figure 41 - Standard ILS View at Decision Height ................................................................ 58

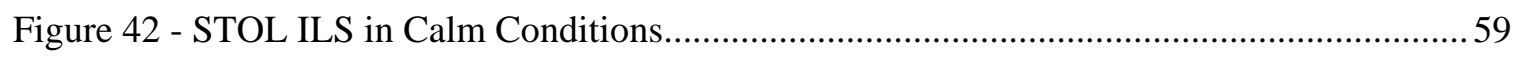

Figure 43 - STOL ILS Glidepath Performance ..................................................................... 60

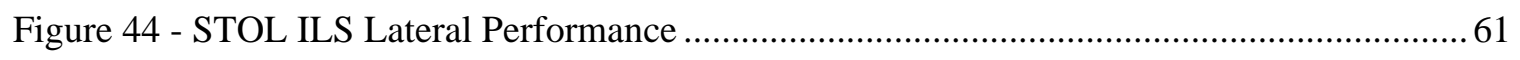

Figure 45 - CTOL SNI Approach with Localizer Coupler....................................................... 62 
Figure 46 - SNI Glidepath Performance with Localizer Coupler................................................ 64

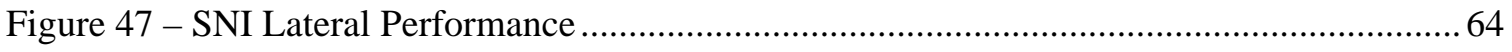

Figure 48 - SNI with Localizer Coupler View at Decision Height ............................................65

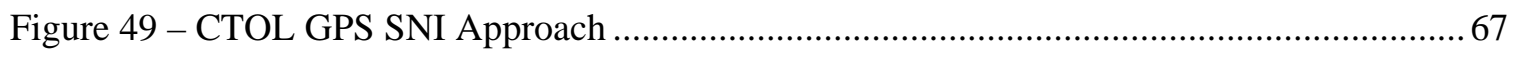

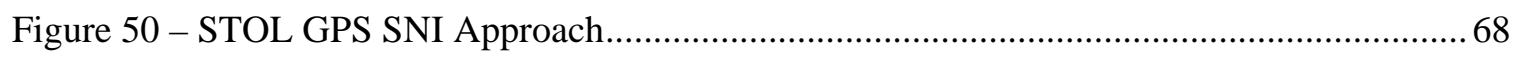

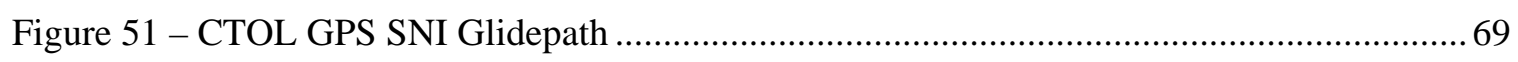

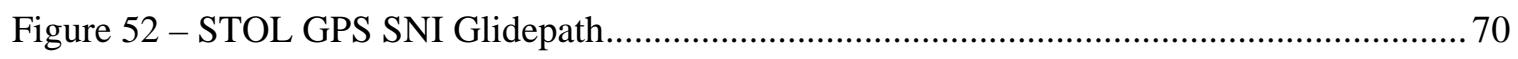

Figure 53 - CTOL GPS SNI View at Decision Height .......................................................... 71

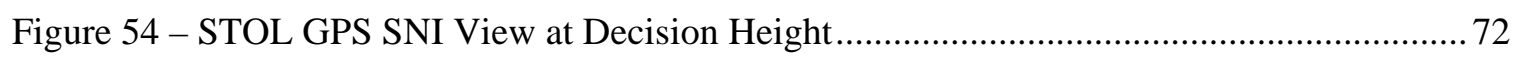




\section{LIST OF ACRONYMS}

\begin{tabular}{|c|c|}
\hline 6DOF & Six Degree-of-Freedom \\
\hline AGL & Above Ground Level \\
\hline AOA & Angle of Attack \\
\hline ATC & Air Traffic Control \\
\hline BLC & Boundary Layer Control \\
\hline CCW & Circulation Control Wings \\
\hline CDI & Course Deviation Indicator \\
\hline CTOL & Conventional Takeoff and Landing \\
\hline $\mathrm{DH}$ & Decision Height \\
\hline DLC & Direct Lift Control \\
\hline DME & Distance Measuring Equipment \\
\hline $\mathrm{EBF}$ & Externally Blown Flaps \\
\hline FAA & Federal Aviation Administration \\
\hline FMS & Flight Management System \\
\hline GPS & Global Positioning System \\
\hline GPSS & GPS Steering \\
\hline HSI & Horizontal Situation Indicator \\
\hline IFR & Instrument Flight Rules \\
\hline ILS & Instrument Landing System \\
\hline IMC & Instrument Meteorological Conditions \\
\hline INS & Inertial Navigation System \\
\hline NASA & National Air and Space Administration \\
\hline OB & Outboard (flaps) \\
\hline PCAS & Pitch Control Augmentation System \\
\hline PID & Proportional-Integral-Derivative controller \\
\hline PolyQSRA & The Cal Poly Implementation of the QSRA simulation \\
\hline QSRA & Quiet Short-Haul Research Aircraft \\
\hline RNP & Required Navigation Performance \\
\hline SAS & Stability Augmentation System \\
\hline SNI & Simultaneous, Non-Interfering \\
\hline STOL & Short Takeoff and Landing \\
\hline SWAT & Students With Attitude \\
\hline USB & Upper Surface Blowing \\
\hline VMC & Visual Meteorological Conditions \\
\hline VOR & Very-high frequency Omni-directional Range \\
\hline
\end{tabular}




\section{INTRODUCTION}

To the best of the author's knowledge, work to create a Short Takeoff and Landing (STOL) flight simulator at Cal Poly, San Luis Obispo began in 2004 with the Students With Attitude (SWAT) team under the direction of Professor David W. Hall with funding from the National Air and Space Administration (NASA). In that year, efforts centered on rudimentary efforts to simulate a ground up designed STOL aircraft (the Model 114), and on checking simulation points against the aerodynamic data available in the Quiet Short-Haul Research Aircraft Phase II Flight Simulation Mathematical Model - Final Report (1).

The next year's SWAT simulation work consisted of attempting to linearize the data from the QSRA Math Model by making mathematical curve fits to the data contained in the aerodynamic tables. The idea was to then integrate the linearized/curve-fit data into the existing Matlab Simulink based flight simulator at Cal-Poly, nicknamed Pheagle. This effort proved more difficult than expected, and to the author's knowledge did not produce a meaningful simulation. Significant issues made this approach ineffective: QSRA aerodynamics data presented difficulties during attempts at linearization, and the Cal Poly Pheagle simulation uses conventions entirely different from the NASA simulation.

The author came to this project during the summer of 2006 with the task of putting together a full featured flight simulator for the QSRA. It became quickly apparent that using the previous attempts at linearizing the aerodynamic data would be insufficient and inaccurate, especially if the model was to cover the entire flight envelope. This led to the decision to digitize all of the aerodynamic data in the QSRA Math Model figures into tabular form. The majority of this work was done over the course of the next year by Cassy Anthony and Natalia Sanchez. Tables were formatted in a manner that could be read-in and accessed with a table lookup by the Pheagle flight simulator. 
During this time, the author's efforts were centered mostly on replicating QSRA control system diagrams in Matlab Simulink blocks. The QSRA Math Model specifies the engine model as well as the flight control system by use of control diagrams. These completed control systems were eventually combined with the digitized aerodynamics data and the Pheagle simulator, which in theory should have resulted in a complete flight simulator.

After months of troubleshooting, it became apparent that this effort was plagued by many critical issues. Foremost of these issues was the lack of standardization between the Pheagle simulator and the QSRA Math Model. Variations in coordinate systems, units, and sign conventions made validation of this simulator nearly impossible. Related to this issue was a lack of detailed record keeping for variables up to this point. Other major issues were encountered with the Pheagle flight simulator itself. Pheagle was not capable of "throwing” meaningful errors when reading the aerodynamics tables, nor was it capable of showing intermediate values that were occurring in the aerodynamics calculations. Pheagle’s six degree-of-freedom (6DOF) model had become a black box that was crunching numbers, but not giving meaningful results.

In mid-2008, the decision was made to divorce the QSRA efforts from the Pheagle simulator. Starting with a blank Simulink workspace, the 6DOF model specified in the QSRA Math Model was implemented. The QSRA 6DOF is taken from A Standard Kinematic Model for Flight Simulation at NASA-AMES (2), which proved to be an invaluable reference for setting up the kinematic model. In parallel with this implementation, a Microsoft Access database of variables was developed, with the intention of preventing the issues of units and conventions that plagued previous attempts. This in depth recordkeeping was tedious, but eventually proved invaluable. On completion, the 6DOF model was verified for point masses, with results shown in Appendix I. 
The controls and engine blocks from previous attempts were then integrated into the new implementation using the same Access database recordkeeping procedure. The last step in making the simulator flyable was adapting the aerodynamics data formatted for Pheagle to a native Simulink table lookup format. Pheagle made heavy use of external text files containing large amounts of data that were read in by $\mathrm{C}$ code. This was supposedly faster than utilizing Simulink’s built-in table lookup and provided for greater data portability. However, the current model using Simulink’s built-in ability for over 200 complex table lookups is still capable of running faster than real-time, and data portability is not an issue for this simulator because it is only designed to simulate one aircraft. Using Simulink’s built-in table lookups also allows calculated values to be easily viewed by the end user at any point in the program which, as mentioned before, was problematic in Pheagle.

Attention to detail on this most recent effort paid off: after only a couple of hours of debugging, the QSRA simulator (henceforth referred to as PolyQSRA) was successfully flying. Initial “flight” testing was accomplished using a joystick, and then remaining efforts were centered on designing an autopilot capable of flying the aircraft in all flight conditions, including an approach coupler for both straight in and circling approaches.

NASA's interest in the Simultaneous, Non-Interfering (SNI) approach for commuter class aircraft gave purpose to PolyQSRA. When this project started in 2004, the somewhat ambiguous end goal was to use the simulator for accurate STOL simulations, and possibly be able to alter the simulation as necessary to simulate other aircraft. As the project evolved, the following goals evolved as well:

- Assess ability of current generation autopilots to track a SNI approach course in an IFR environment 
- Assess the challenges of designing approach couplers for IFR use with STOL configured aircraft

- Assess the viability of the SNI approach as an IFR procedure

\section{THE QUIET SHORT-HAUL RESEARCH AIRCRAFT}

The Quiet Short-Haul Research Aircraft (QSRA) came into being as a result of a NASA contract competition in 1976 for a STOL research aircraft (3). The competition included a McDonnell Douglas externally blown flap concept, but Boeing's upper surface blowing configuration of the QSRA was awarded the contract in February 1976 (Figure 1).

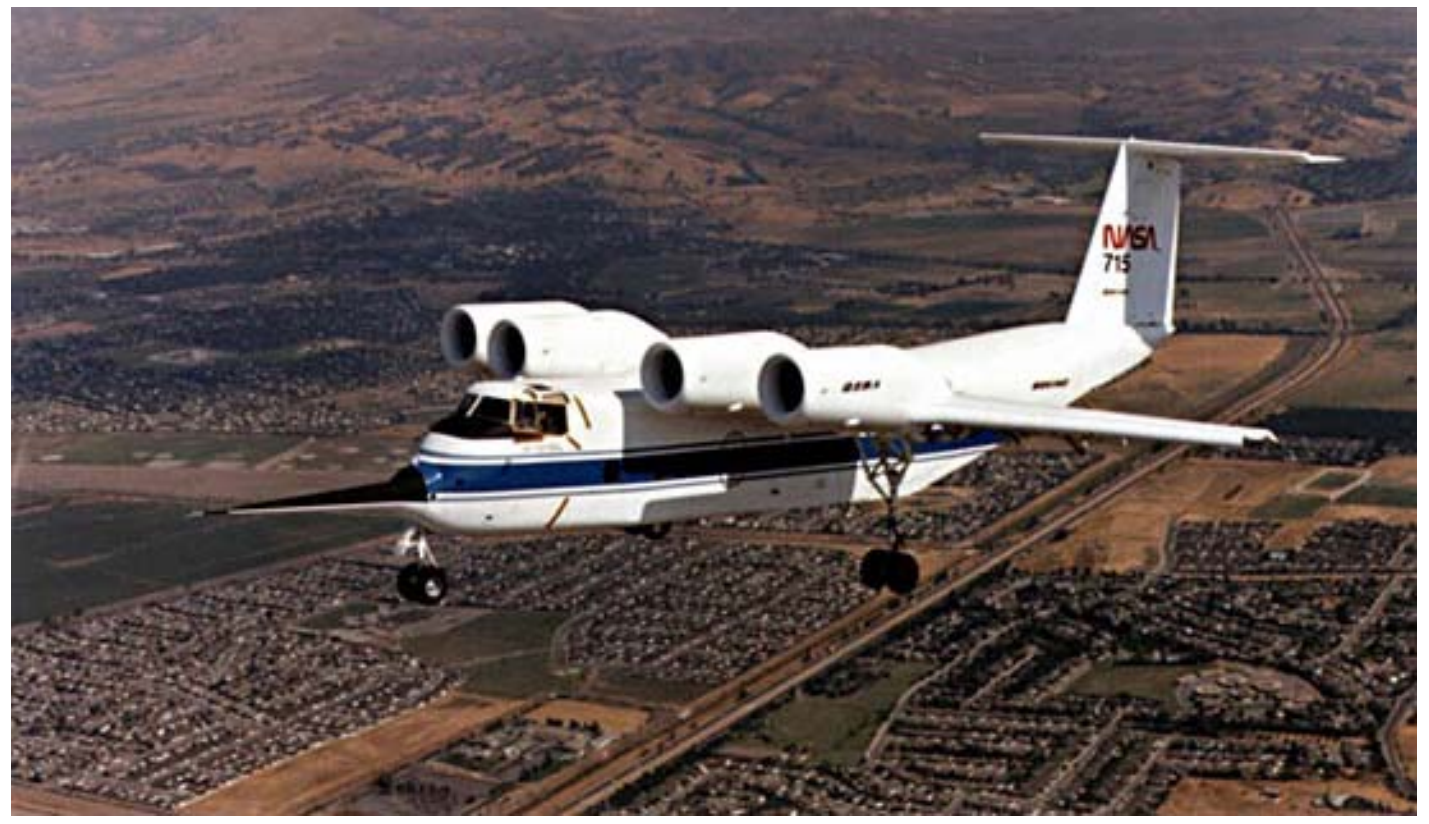

Figure 1 - The NASA QSRA

Boeing created the QSRA by modifying a DHC-5 de Havilland Buffalo (US military designator C-8A) (Figure 2). Originally a turboprop, the QSRA was retrofitted with four Avco-Lycoming YF-102 turbofans on the top surface of the wing, each providing 7,500 lbf sea level static thrust, resulting in a thrust to weight of 0.49 at a gross weight of 50,000 lb and wing loading of $80 \mathrm{psf}$. The QSRA first flew on July 6, 1979. 


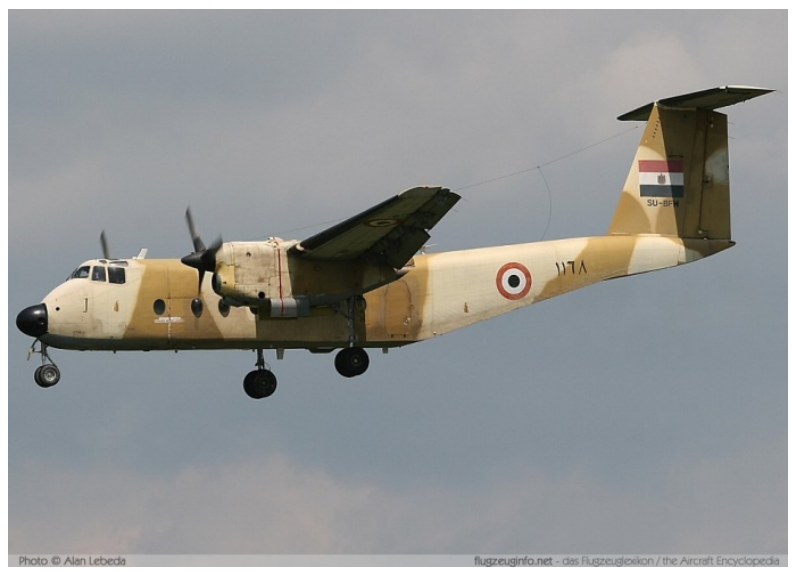

Figure 2 - DHC-5 Buffalo

\section{HIGH LIFT DEVICES}

The QSRA was retrofitted with a heavily modified experimental wing, making the aircraft suitable only for research purposes: for instance, additional fuel was carried in the cargo bay. The wing includes many high lift features, including upper surface blowing/USB flaps, standard outboard flaps, drooping ailerons, and boundary layer control on the wing leading edge and ailerons (Figure 3).

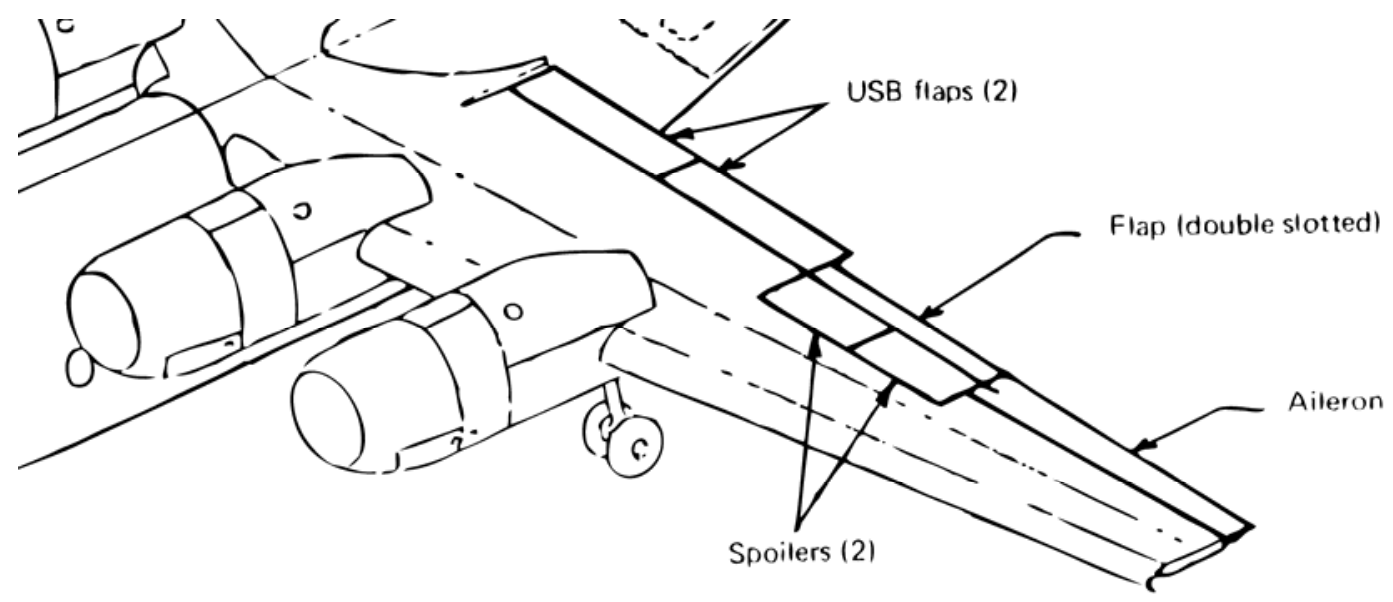

Figure 3 - QSRA Wing (1)

The most prominent high lift element of the QSRA is upper surface blowing (USB). The engines are exhausted over the top surface of the wing by a D-shaped nozzle (Figure 4). The high energy exhaust flow can then be directed / deflected by the pilot's selection of the USB flap position, 
between zero and $66^{\circ}$. Upper surface blowing results in a significant increase in effective lift due to vectored thrust, lower pressure on the top surface, and increased circulation (1).

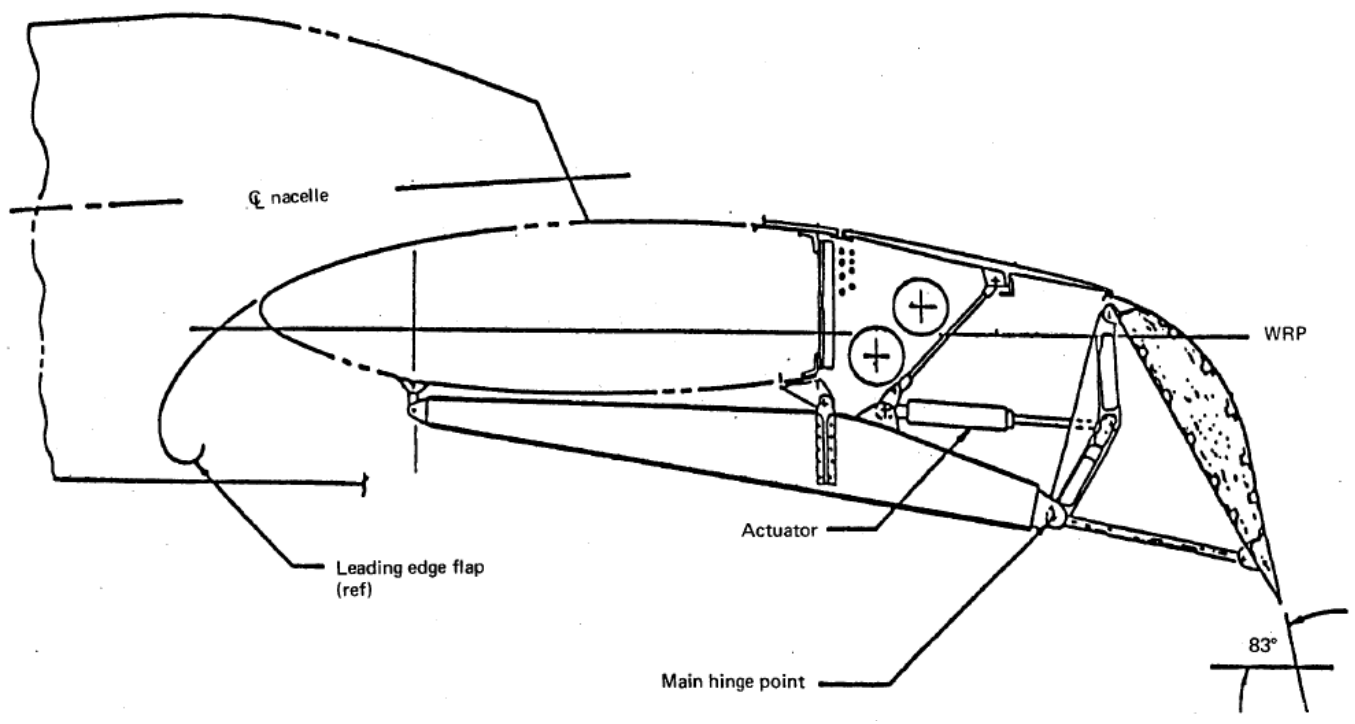

Figure 4 - USB Nozzle (1)

The double slotted outboard flaps and drooping ailerons are more conventional in nature (Figure

5). The outboard flaps can be extended up to $59^{\circ}$, which mechanically results in a $22^{\circ}$ droop in both ailerons. In their un-drooped configuration, the ailerons have a travel of $\pm 20^{\circ}$. While drooped, the ailerons deflect between zero and $50^{\circ}$ (positive down) (1).

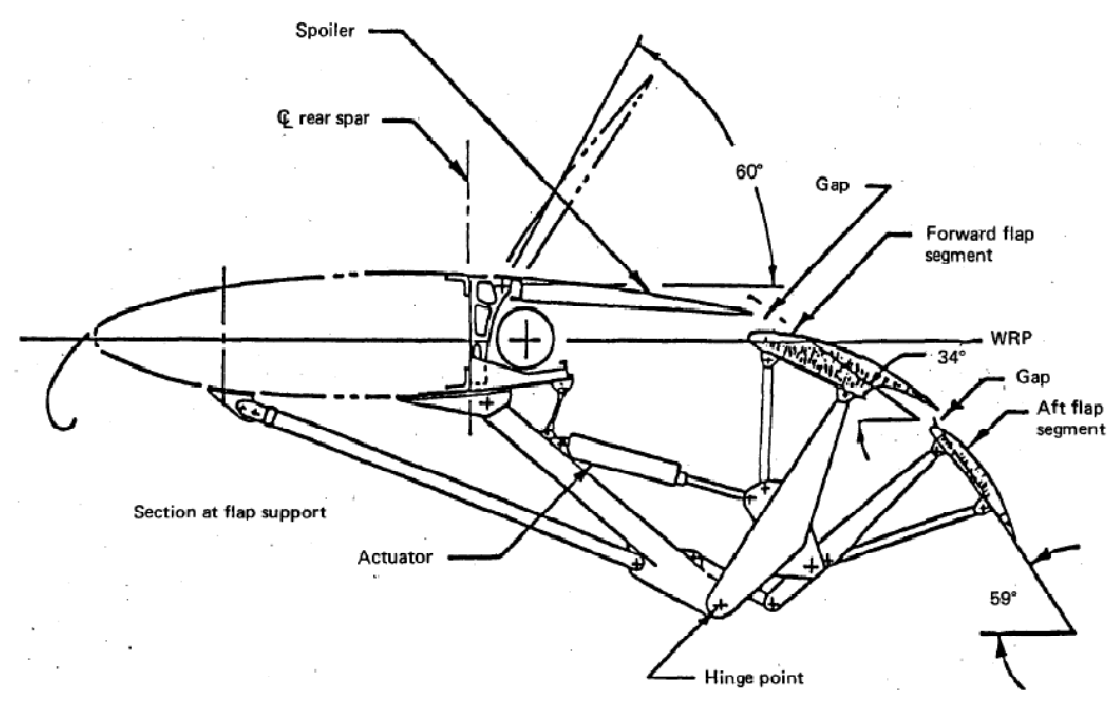

Figure 5 - Outboard Flaps (1) 
The QSRA’s Boundary Layer Control (BLC) system consists of two sets of ejectors: one set along the wing leading edges, and a second set forward of the ailerons. The leading edge BLC is designed to delay wing stall in high angle-of-attack / low dynamic pressure situations by promoting boundary layer attachment. The aileron BLC serves the purpose of increasing aileron effectiveness at low dynamic pressures. The system utilizes high pressure bleed air from the YF102 turbofans. As shown in Figure 6, bleed air is cross-ducted: the left engines power the BLC for the right wing and vice-versa. This ensures that during an engine out, BLC and engine thrust are not lost on the same side simultaneously. The outboard engines power the aileron BLC, and the inboard engines power the leading edge BLC. In the event of an inboard engine failure, a check-valve opens to provide partial blowing to both leading edges. In the event of an outboard engine failure, the opposite aileron is not provided with any blowing. To provide an appropriate blowing mixture, an ejector pump mixes approximately $10 \%$ compressor core bleed with $3 \%$ fan bleed (1).

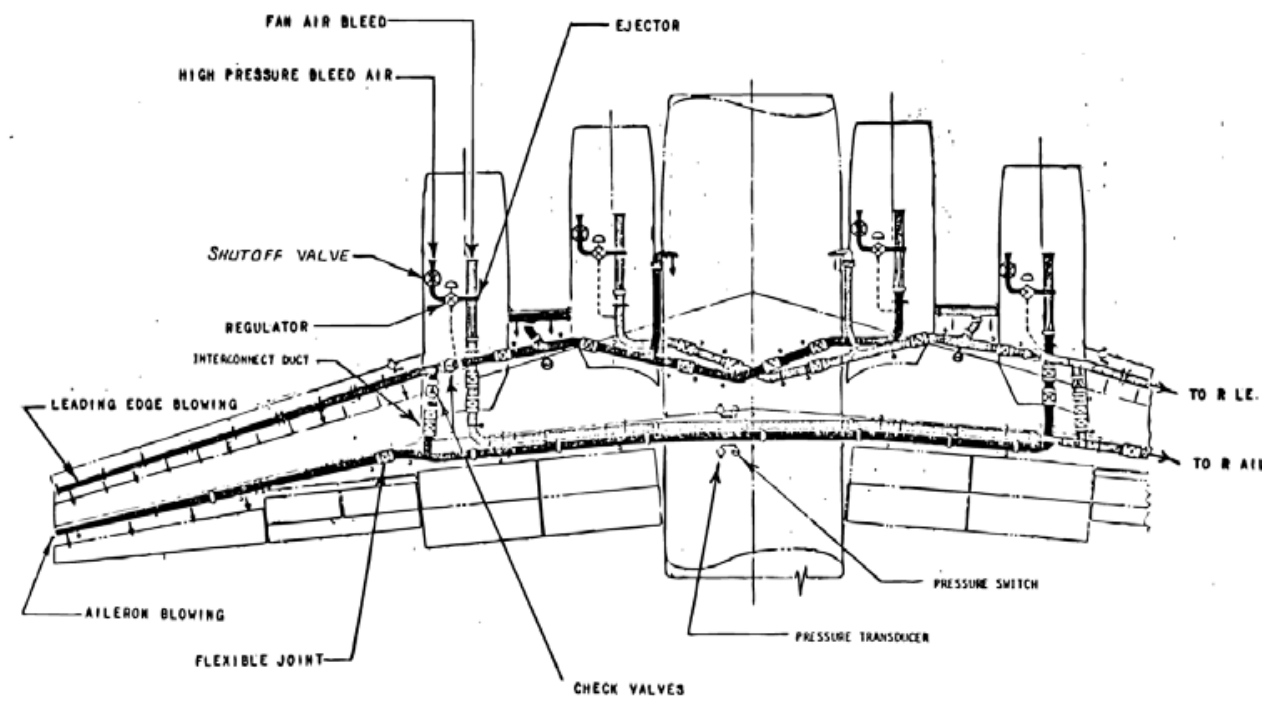

Figure 6 - BLC System and Cross-Ducting (4)

The combination of all of these high lift devices allows the QSRA to achieve a maximum $C_{L}$ of 5.4 at 65 knots; maximum lift coefficients in excess of 10 were demonstrated in other conditions 
(3). Landing distance for the QSRA from a 7.5 approach at 65 knots with all engines and high lift operational is $1,500 \mathrm{ft}$, including the standard safety factor of 1.66 (4).

\section{FLIGHT CONTROLS}

The following section will discuss flight controls on the actual QSRA test vehicle. The QSRA utilizes all hydraulic flight control surfaces. Though there is no direct mechanical connection between the flight control surfaces and the pilot's yoke and pedals, the QSRA is not a true fly-bywire aircraft. Instead, primary flight control inputs are sent by mechanical cable linkages to an artificial feel unit, which in turn utilizes more mechanical linkages to transmit desired flight control position to the hydraulic actuators at the flight control surfaces. Additional actuators are placed inline with the flight control linkages to add stability and control augmentation to flight control inputs. A general schematic of the flight control system is shown in Figure 7.

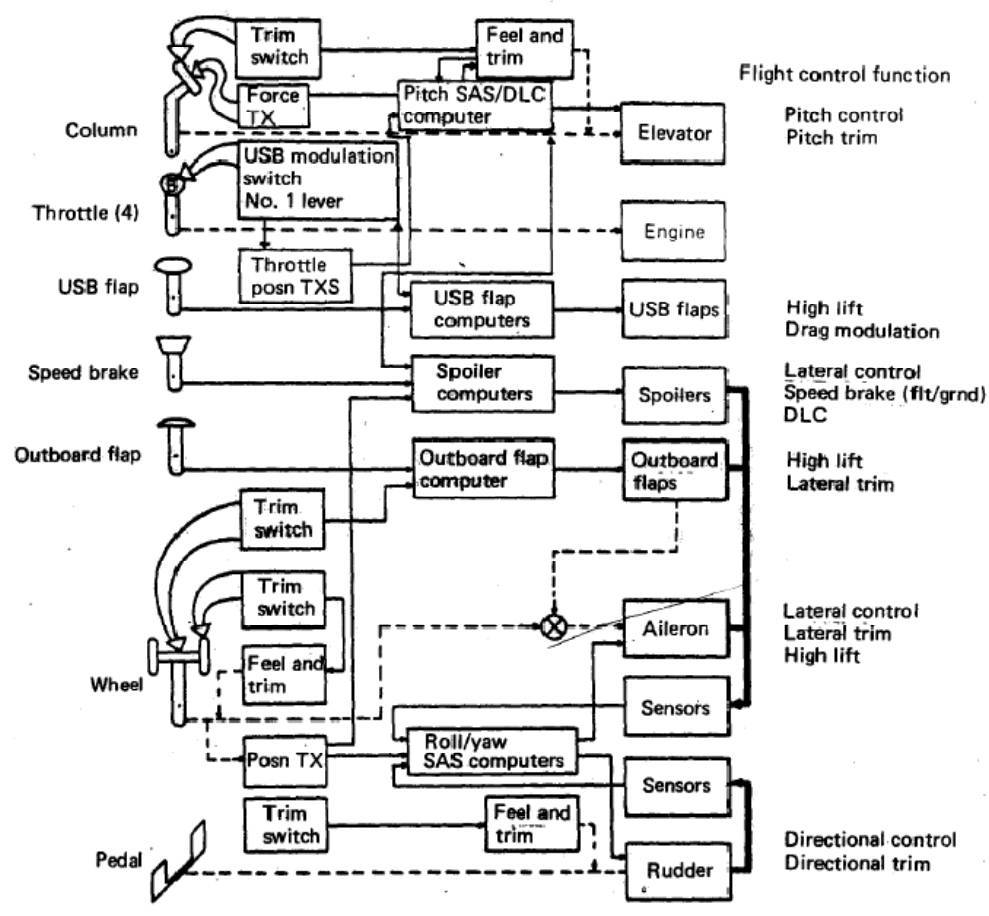

Figure 7 - Flight Control System Schematic 
The longitudinal control system of the QSRA utilizes an elevator powered by a dual tandem hydraulic actuator. The feel and centering unit is located in the empennage close to the actuators. Column position is mapped directly to a desired position of the elevator. Elevator trim is accomplished by moving the center position of the feel system; applying up elevator trim will move the center position of the column back. To provide realistic control forces, the feel system is designed to vary the column force gradient with dynamic pressure. In the event of a full hydraulic failure, manual reversion of elevator control is accomplished using a servo tab and jackscrew trim, both of which are locked in “neutral” for standard operations.

A Pitch Control Augmentation System (PCAS) is utilized to reduce the longitudinal workload on the pilot, especially in the powered lift configuration. A load cell built into the pilot's control yoke senses control force applied by the pilot, which is matched to PCAS pitch rate. When the pilot is not applying force to the control yoke (the yoke is in-detent) the PCAS maintains last selected pitch attitude, making the QSRA's PCAS an attitude hold control system. This is particularly useful for a stabilized STOL approach, which is flown with precise attitude control.

The QSRA’s ailerons and rudder are likewise powered by dual tandem hydraulic actuators, and they receive their commands through cables with artificial feel systems. The lateral and directional control systems utilize simpler feel systems: controls are driven to center by a centering bungee, and the center position of the bungee can be changed by applying aileron or rudder trim. Lateral and directional Stability Augmentation Systems (SAS) are utilized to damp a significant Dutch roll tendency. Despite the obvious lateral/directional coupling in the aircraft dynamics, the specifications for the SAS damping are uncoupled.

The outboard flaps and the USB flaps are all hydraulically actuated. The outboard flap deflection is electronically controlled by a single lever in the cockpit. An outboard flap trim switch is 
provided for engine out situations: if the outboard flaps are extended more than $30^{\circ}$, a momentary selection of the flap trim (left or right) retracts the flap on the side selected to $30^{\circ}$ to assist in the desired rolling moment. The USB flaps are electronically controlled by a combination of a lever in the cockpit and a three position switch mounted on the number 1 throttle. Full deflection of the cockpit lever results in a USB flap command of $30^{\circ}$. Engineers recognized that USB flap deflections in excess of $30^{\circ}$ would result in significant pitch/power coupling, so USB flap extensions between $30^{\circ}$ and $66^{\circ}$ are commanded by the switch on the throttle, allowing the pilot to modulate flap selection and power as necessary without significant hand gymnastics.

The spoilers on the QSRA serve three control functions: speedbrakes, roll augmentation, and Direct Lift Control (DLC). The location of wing spoilers can be seen in Figure 3. The spoilers are electronically controlled, hydraulically actuated, and deflect to a maximum of 60 degrees. The speed brake function is controlled by a lever in the cockpit and allows the pilot to command spoiler deflection in flight to prevent speed buildup in descents and on the ground to provide a "lift dump” after landing. Roll augmentation is provided by differential deflection of the spoilers, resulting in a differential loss of lift. Spoiler pickup for roll augmentation occurs at approximately 40\% wheel travel. The spoiler Direct Lift Control function is designed to speed up aircraft response to throttle inputs in the powered lift configuration. When DLC is selected by the pilot, the spoilers are symmetrically deflected to $13^{\circ}$. Changes in the throttle command then result in a DLC spoiler command: an increase in throttle results in a temporary decrease in spoiler deflection, resulting in a quicker throttle response. A washout function returns the spoilers to $13^{\circ}$ deflection.

\section{SIMLUTANEOUS NON-INTERFERING APPROACH}

NASA's vision for future air travel involves the use of STOL aircraft to provide increased pointto-point service to airports otherwise too small for commercial airline service. This not only has 
the potential to increase overall transportation efficiency, but shows promise in reducing the runway crowding at traditional hub-and-spoke airports. STOL aircraft would of course still utilize traditional airports; for this to be viable, STOL aircraft would need to utilize airspace and runways at busy airports that is currently unused, thus marginal traffic load would be added to currently utilized airports. Hence, the concept of the Simultaneous, Non-Interfering (SNI) approach has been developed, with the intention of utilizing runways and airspace that otherwise would go un-utilized.

The SNI approach is still in the conceptual phase, but the general idea is that the STOL aircraft flies an approach above the Conventional Takeoff and Landing (CTOL) aircraft, arrives high at the runway threshold, and then performs a descending, circling maneuver (akin to a military overhead break). The STOL aircraft then arrives at a decision altitude of 100-250 feet and on runway centerline and glidepath. An example of a conceptual SNI profile is shown in Figure 8.

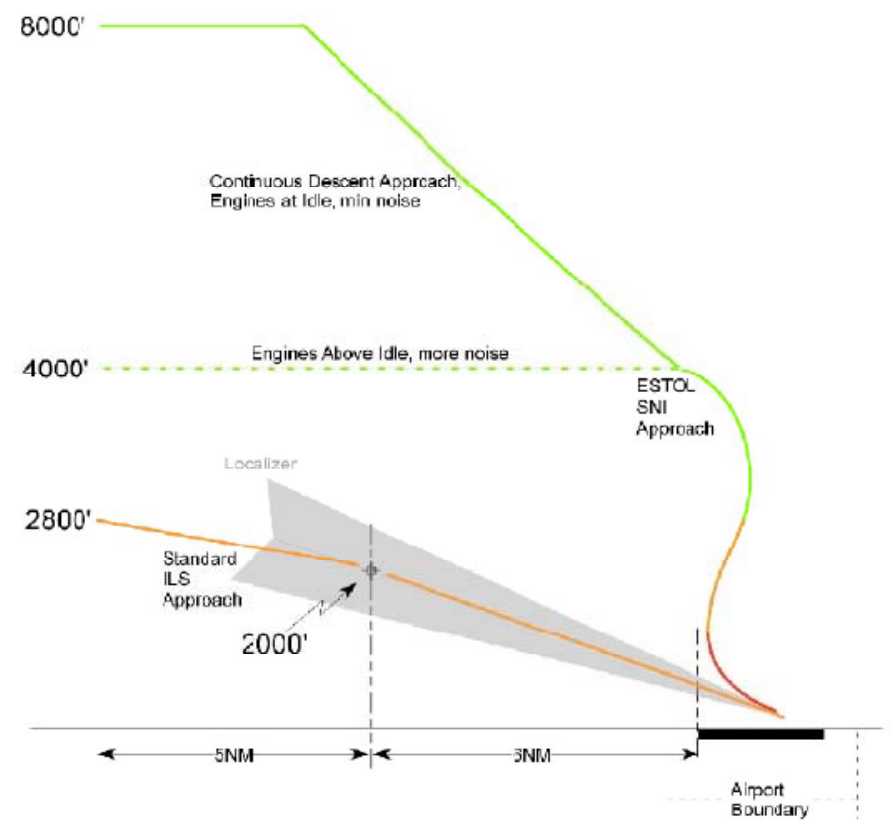

Figure 8 - SNI Approach Profile (5) 
Figure 9 depicts Dallas Fort-Worth airport, showing where an SNI approach could increase airport utilization. SNI approaches could be flown to runway 31L while approaches were simultaneously being flown to runways 36L or 36R. The aircraft on the SNI approach would have adequate vertical separation from aircraft performing conventional approaches on 36L and 36R up until the time the circling maneuver was initiated, at which point lateral separation should be adequate.

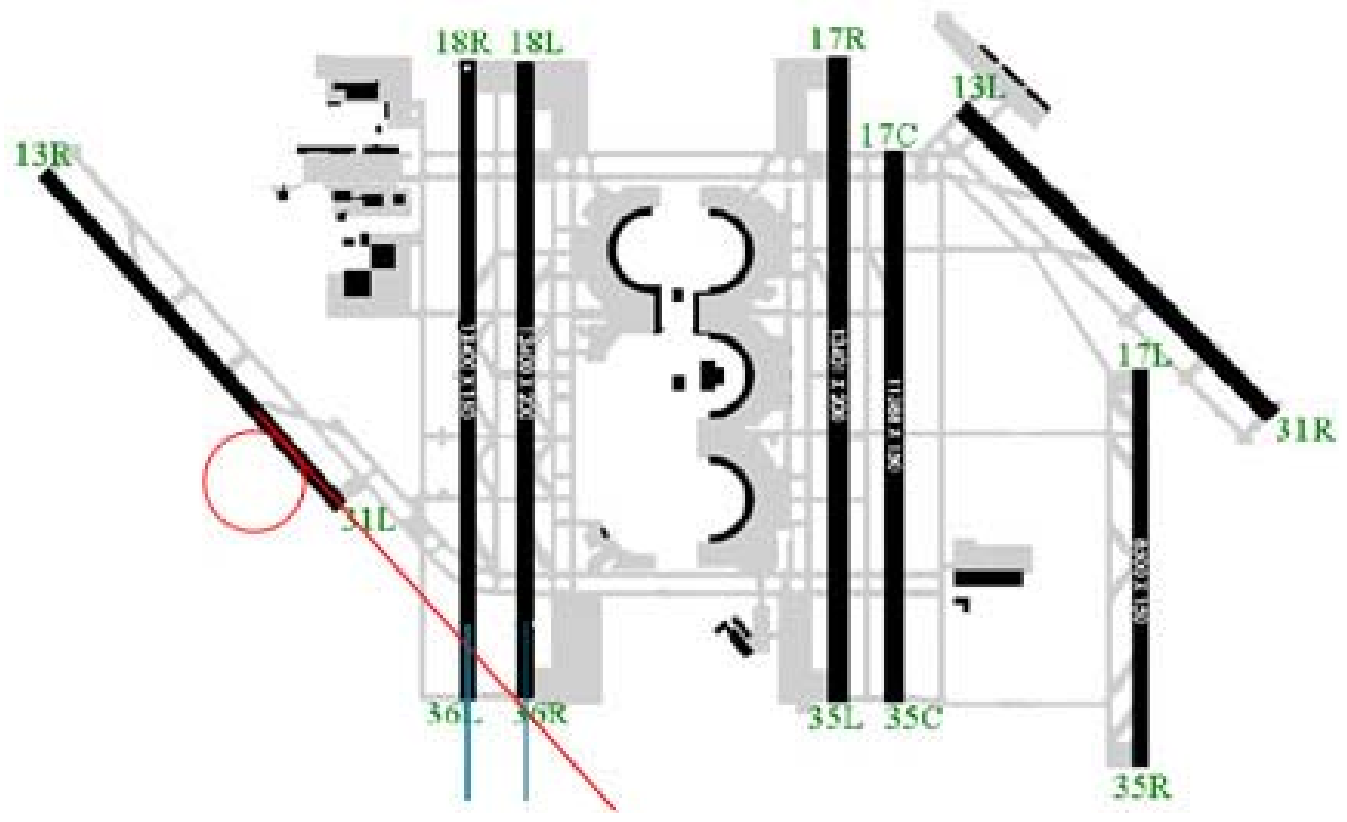

Figure 9 - DFW SNI Approach Example (6)

Noise is another concern for STOL operations, possibly making a SNI approach useful or necessary even at satellite airports in order to reduce the noise impact on the surrounding communities. STOL aircraft utilizing powered lift such as USB, Externally Blown Flaps (EBF), and Circulation Control Wings (CCW) fly approaches at significantly higher than normal power settings. Additionally, at any power setting, the concepts that allow STOL flight result in inherently higher noise levels when compared to CTOL aircraft. Compounding the issue are the lower approach speeds used by STOL aircraft, which would result in increased noise saturation due to increased overflight time when compared to a conventional approach. A flight test jointly 
conducted by NASA, the United States Air Force, and Cal Poly San Luis Obispo with a C-17 at Roger's Dry Lakebed produced the noise comparison shown in Figure 10. This flight test successfully demonstrated the SNI approach’s effectiveness at keeping most of the worst noise saturation near the airport boundary (5).
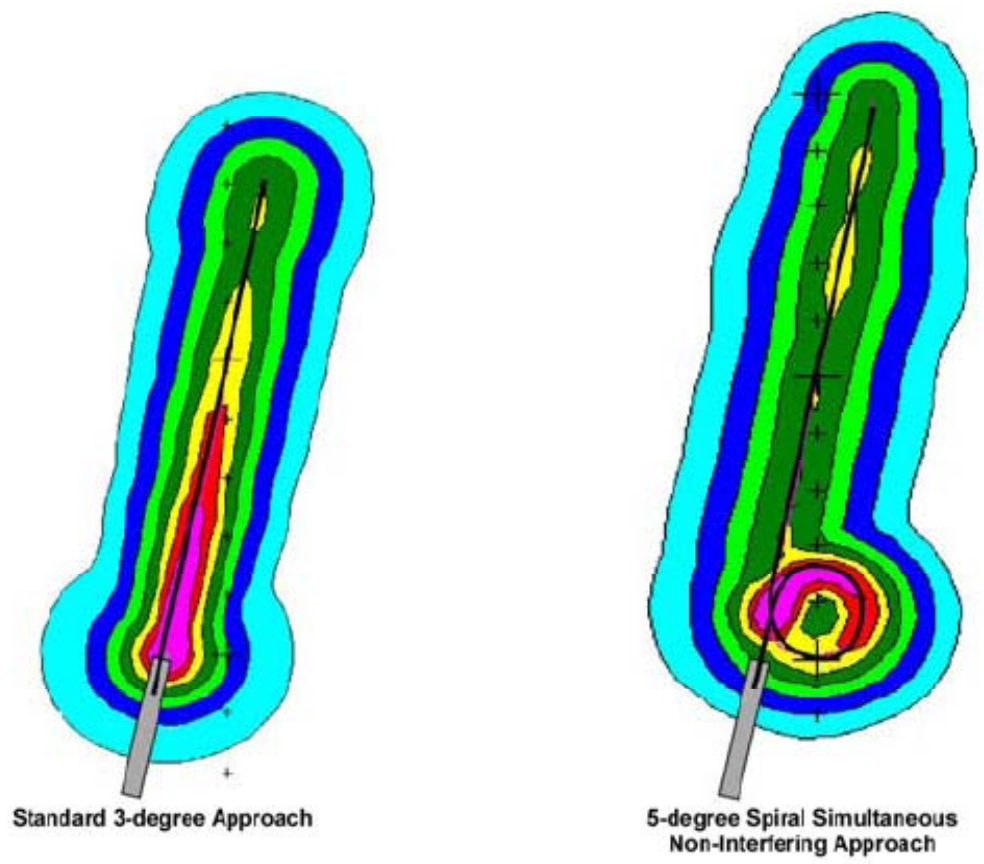

Figure 10 - Standard and SNI Approach Weighted Noise Contours (5)

As the SNI approach is still in the conceptual phase, it is not entirely clear what the parameters of the approach should be. Traffic flow and noise considerations seem to dictate that the aircraft decelerate during the descending spiral. This would allow the initial straight segment to be flown with a speed of 120-150 knots with a reduction to 65-90 knots for landing. This would both prevent Air Traffic Control (ATC) bottlenecks and also reduce noise saturation in the outlying areas.

As the SNI approach is still in the development stage, it is not entirely clear how the deceleration should be accommodated. One concept utilizes a constant bank angle with a uniform 
deceleration, resulting in a constant load factor and a funnel shaped descending spiral, described mathematically by an Ouija curve shown in Figure 11 (5). The advantage of this approach is that, theoretically, it would be easiest to fly because bank angle is constant. Furthermore is should be most comfortable to passengers because load factor is constant. Hange suggests that the uniform longitudinal deceleration would be on the order of $0.1 \mathrm{Gs}$.

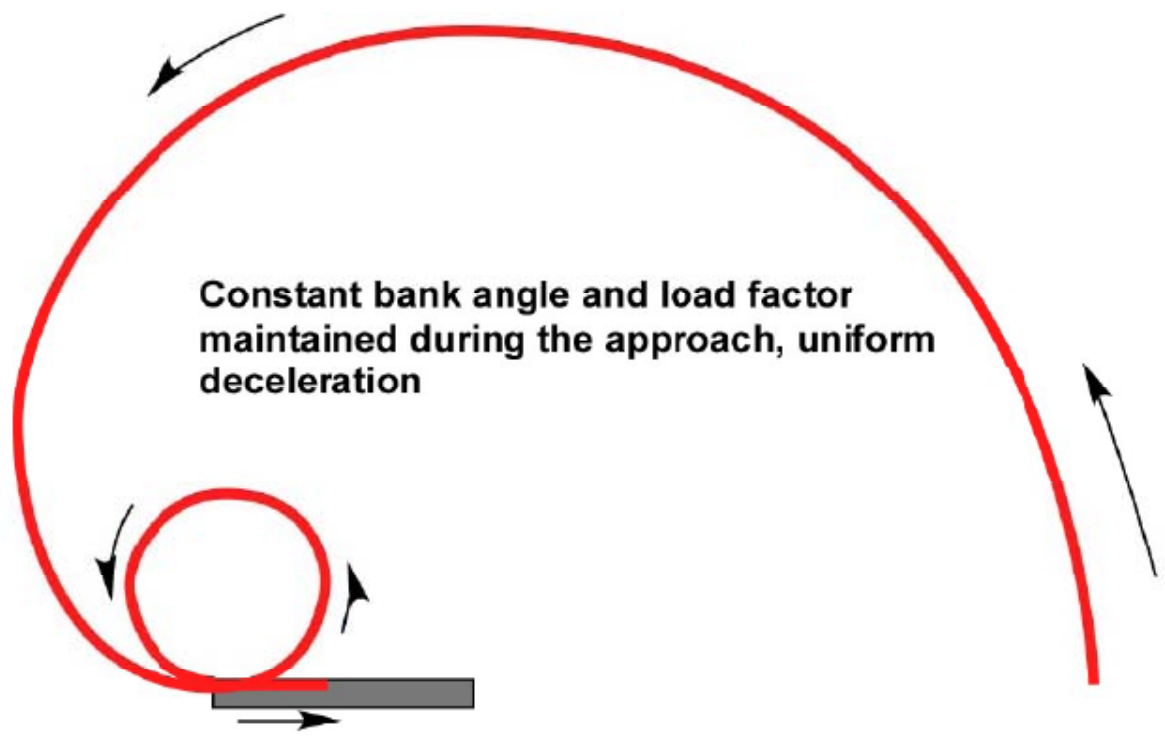

Figure 11 - The Ouija Curve Approach Trajectory (5)

Many elements of this approach still need to be worked out. First off, aircraft deceleration is seldom uniform. Activation of high lift devices necessary for slower flight (flaps, etc.) typically results in a significant transient longitudinal deceleration; it would be quite a challenge to make the deceleration uniform throughout the approach. Also, the idea of constant bank angle/load factor breaks down with any wind. Bank angle would have to be varied in order to make wind corrections, or an unpredictable approach path would result. An unpredictable approach path is probably not feasible because the path might not end at the runway, and also because ATC would not know ahead of time the path each aircraft would take. Along the same lines, it is not clear at this point if aircraft with different approach speeds would follow the same Ouija approach path. 
Another SNI concept involving a continuous radius spiral is probably more feasible. This approach will be referred to as the continuous descent SNI approach (Figure 12). The STOL aircraft would remain quite high until the standard localizer outer marker, and then would start an aggressive power off descent at cruise speeds. This ensures the minimum noise footprint and should minimize traffic conflicts, at least for inbound traffic. At the beginning of the spiraling turn, the aircraft is smoothly decelerated and reconfigured for the STOL landing.

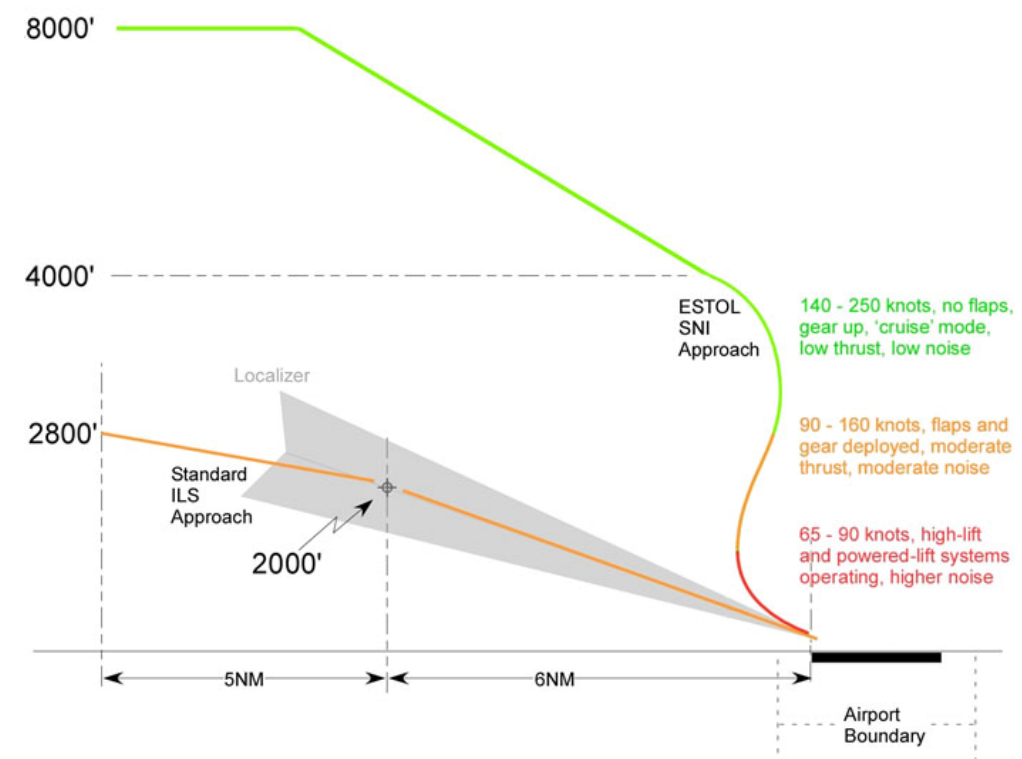

Figure 12 - Aggressive Continuous Descent SNI Approach (7)

This type of approach was flight tested during the 2005 NASA, Air Force, and Cal Poly SLO noise measurement flight test with the C-17. The flight test, however, was flown at a constant speed of $120 \mathrm{kts}$. Using a C-17 simulator, a method to fly the SNI approach on autopilot had been devised; however, during the flight test, a greater than expected crosswind was experienced resulting in less than acceptable autopilot performance. The approaches were instead hand flown with bank angle corrections given by a flight test engineer using a laptop computer. The C-17 approaches were flown with a radius of 0.75 miles. Ground tracks from three separate approaches are shown in Figure 13 (5). 


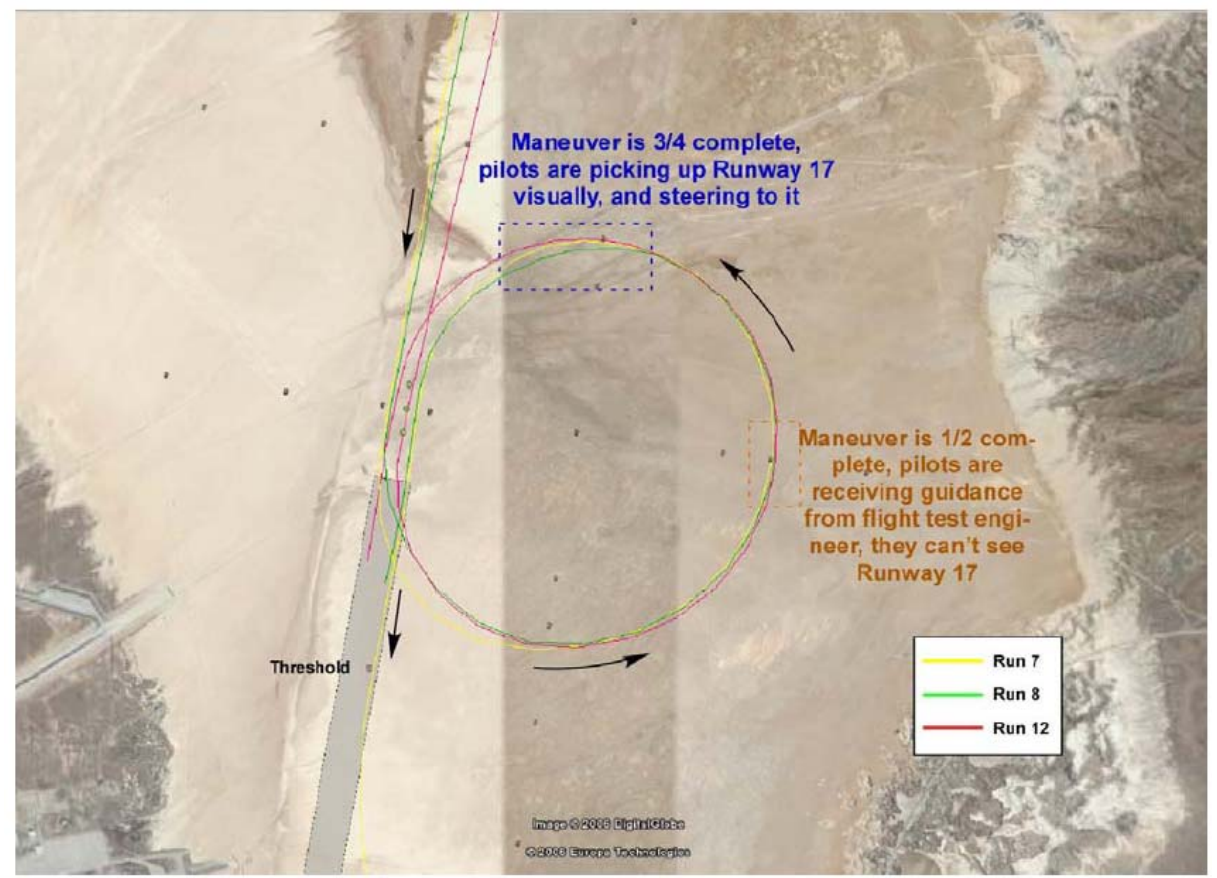

Figure 13 - C-17 SNI Approach Ground Tracks (5)

Though the flight test demonstrated ground track repeatability for hand flown approaches (autopilot approaches did not produce an acceptable ground track), there are operational, navigational, and regulatory issues that still must be addressed for the success of the SNI approach. These approaches were flown in Visual Meteorological Conditions (VMC) and as such it was relatively easy for the pilots to maintain orientation. To integrate seamlessly into the ATC environment, the SNI approach must be an Instrument Flight Rules (IFR) procedure that could be flown in Instrument Meteorological Conditions (IMC). Flight path must be precise enough to put the aircraft on the runway centerline at the termination of every approach as well as prevent encroachment into other airspace. For the pilot this means new visual flight guidance systems, and in the author's opinion, a requirement that the approach be flown with use of autopilot.

Another major issue is that the SNI approach violates the FAA's stabilized approach concept. Regulations currently prohibit the pilot from continuing an approach below 1,000 ft Above Ground Level (AGL) in IMC or $500 \mathrm{ft}$ AGL in VMC if the aircraft is not established on runway 
centerline, fully configured for landing, on glide path, and on speed/momentum target (5). These requirements would have to be revisited is the SNI approach is to be viable. On the other hand, the requirement for a stabilized approach exists for a good reason: transport category aircraft carry a lot of momentum, and if it isn't pointed in the right direction at 500 or $1,000 \mathrm{ft}$ respectively, there is an unacceptable probability of an unsuccessful outcome if the approach is continued. This again leads the author to believe that for SNI approach implementation to be successful, it must be flown with an autopilot capable of delivering the aircraft on target from every approach.

\section{AUTOPILOT TECHNOLOGY}

The SNI approach presents many new challenges in terms of both autopilot and pilot performance. For the sake of comparison, consider the standard Instrument Landing System (ILS) approach, illustrated in Figure 14. The ILS approach is today's current "golden standard" of safety and precision for instrument approaches. Lateral guidance is provided by a VHF radio azimuth transmitter positioned at the far end of the landing runway; vertical guidance is provided by a UHF azimuth transmitter positioned to the side of the runway about 1,000 feet past the landing threshold. Aircraft navigate or are vectored by ATC to intercept the localizer and glidepath outside the outer marker, which is positioned about 6 nautical miles from the runway threshold. The resulting approach is stabilized on glidepath and localizer for at least six miles. Minimal, if any, aircraft configuration or speed changes are made inside the outer marker. 


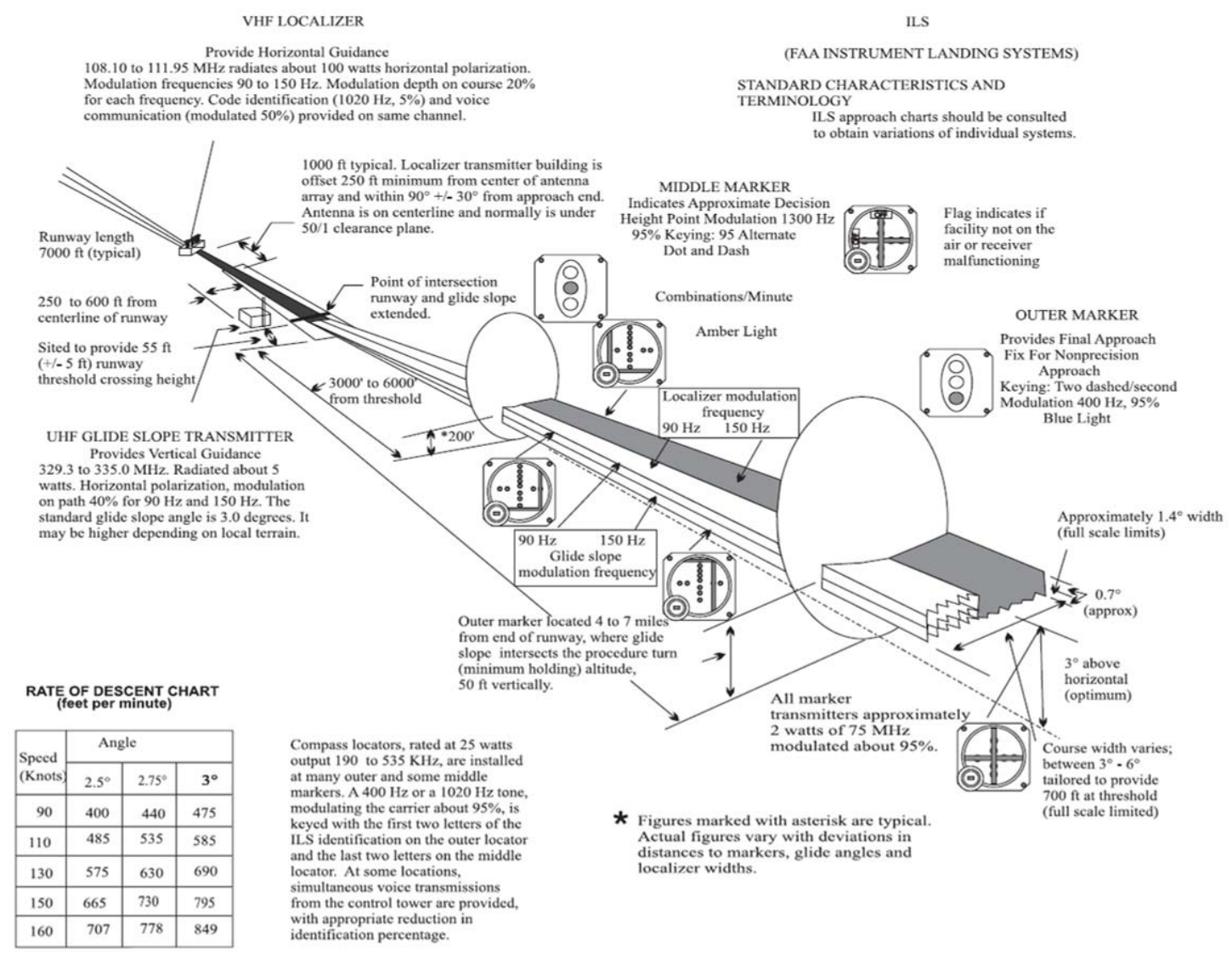

Figure 14 -Standard ILS Approach (8)

The ILS approach provides the "ideal” conditions for finding the runway. The long straight-in gives plenty of time to tweak crosswind corrections and fine tune the approach power setting based on head- or tailwind. The fact that the azimuths converge at a point at the runway is actually quite useful and intuitive: when the aircraft is outside the outer marker making localizer and glideslope intercept, navigation guidance is not overly sensitive. As the aircraft gets closer to the runway, only small corrections are required.

For autopilots and professional pilots alike, the ILS approach is certainly the easiest instrument approach to fly. The first couple of miles on the approach are spent determining proper crosswind and glidepath corrections. Then, aside from corrections for wind shear, the approach practically flies itself. As the aircraft lands, it passes the glidepath transmitter first: glidepath sensitivity and 
error are driven to infinity. This problem is avoided on standard ILS approaches by utilizing a Decision Height (DH) of $200 \mathrm{ft}$ AGL, beyond which the runway must be in sight to the pilot in order to continue the landing. Some airports and aircraft are authorized for lower landing minimums, ranging down to an ILS CAT IIIC approach which is zero visibility full autoland. To accomplish this, a flare-coupler takes over from the glideslope coupler before the glidepath error becomes unstable.

Various flight guidance systems currently in service are pictured in Figure 15. All of these flight guidance systems are capable of coupling to a navigation path, however they way that they do so varies immensely.

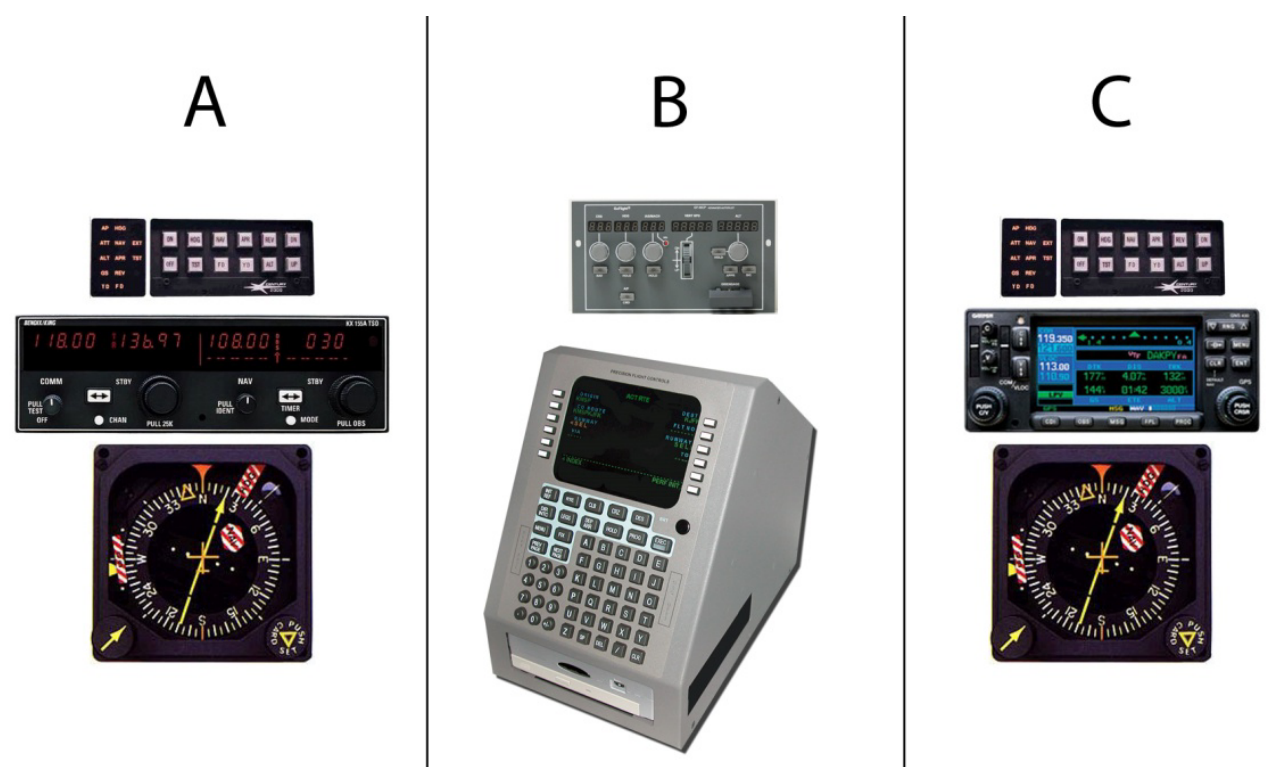

Figure 15 - Example Automatic Flight Systems

The Type-A flight guidance system utilizes a navigation source displayed on the Horizontal Situation Indicator (HSI). The Course Deviation Indicator (CDI) on the HSI (Figure 16) provides right or left of course information to the pilot and can be "swung" by a ground based navigation facility such as VOR or ILS, or by a GPS unit. The autopilot is integrated with the HSI, using the HSI course deviation indicator as a reference for course corrections. The autopilot is only aware 
of the CDI position and the aircraft heading compared to the CDI heading manually selected by the pilot; it is not aware of ground track. A PID controller is utilized to generate a heading command to keep the CDI needle centered. This almost always results in wandering immediately after engagement and after course changes.

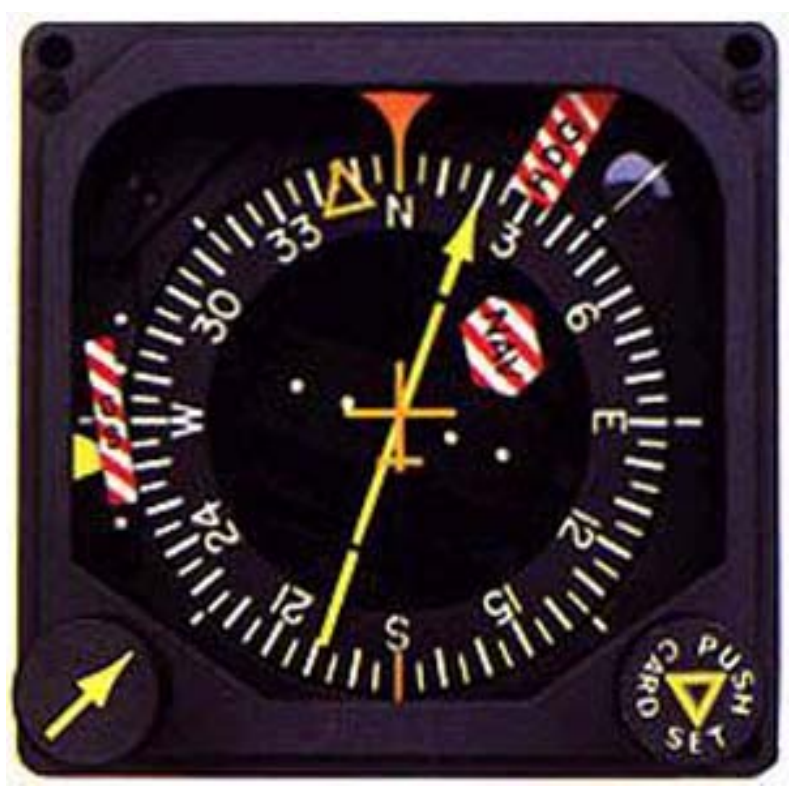

Figure 16 - Example Horizontal Situation Indicator (HSI)

The Type-A autopilot is not particularly well suited to tracking curved courses, though curves with a large enough radius may be tracked successfully: the pilot must manually twist the CDI heading throughout the arc. Though more advanced avionics are becoming more prevalent, many commuter aircraft are still equipped with Type-A flight guidance systems.

The Type-B flight guidance system is known as a Flight Management System (FMS). The FMS is programmed with sequence of waypoints and altitudes for the aircraft to fly, and a ballet of autopilot mode selection will ensure that the desired course and altitudes are flown. Newer FMS units receive their position information from GPS, while older units rely on an inertial navigation augmented by position fixes from ground based stations. Most FMS units are capable of turn anticipation but are not easily programmed with curved segments. Curved segments can be 
approximated by closely spaced waypoints; however, current generation systems are not particularly well suited to providing precise altitude guidance necessary for a precision approach. A PD controller is all that is necessary for heading control; integral compensation is not necessary because the FMS is ground track aware. Most new commuter aircraft come from the factory with FMS flight guidance systems.

The Type-C flight guidance system is similar to Type-A with the exception that the newer generation autopilot/GPS combination is capable of GPS Steering (GPSS). GPSS is a navigation mode where the GPS sends turn commands directly to the autopilot, making the autopilot and GPS combination function more like a FMS. The Type-C flight guidance system is ground track aware and is capable of turn anticipation. Many GPS units natively support flying DME arcs on approaches. However, these arcs are typically at least 8 nautical miles in radius and are only flown to a precision of \pm 1 mile. The Type-C system is a relatively inexpensive upgrade option available to some aircraft with Type-A systems currently installed.

Regardless of the type of automatic flight system onboard the aircraft, all systems revert to PID controllers when engaged in coupled approach mode for an ILS approach. The ILS signal is always primary for navigation of the ILS approach; the autopilot does not use ground track information even if it is available.

The PID controller is particularly effective for tracking the localizer and glideslope. The proportional gain is most active prior to intercept, providing command towards the deflected CDI or glidepath (though it should be noted that some glidepath couplers will only engage from level flight and as the aircraft moves exactly through glidepath center). The properly tuned derivative gain serves to smooth the intercept and prevent overshoots. The proportional gain kicks in last 
and settles on any steady state wind correction required. Proportional gain is zero for the glidepath coupler; there is never a need for a steady state pitch rate.

One of the main questions that this thesis attempts to answer is whether it is possible for readily available autopilots to track the curved path of the SNI approach, and if not, what modifications would be required. Given that current autopilots are only capable of tracking a precise glideslope in coupled approach mode, the test is at the mercy of the adaptability of the PID controller to the curved path.

The question then becomes how to define autopilot performance as adequate. The Federal Aviation Regulations provide some guidance as to autopilot performance requirements. 14 CFR $\S 25.1329$ (see Appendix II) regarding certification of flight guidance systems for transport category aircraft does not specifically address coupled performance, but does state that autopilot use may cause no more than a "minor transient" (9).

The certification requirements for obtaining a CAT II ILS authorization 14 CFR Appendix A to Part 91) provide more useful guidance. At least 50 ILS approaches must be demonstrated to a 100 foot decision height with a 90\% success rate. A successful approach is defined as follows (9):

(i) At the 100-foot decision height, the indicated airspeed and heading are satisfactory for a normal flare and landing (speed must be plus or minus 5 knots of programmed airspeed, but may not be less than computed threshold speed if autothrottles are used);

(ii) The aircraft at the 100-foot decision height, is positioned so that the cockpit is within, and tracking so as to remain within, the lateral confines of the runway extended;

(iii) Deviation from glide slope after leaving the outer marker does not exceed 50 percent of full-scale deflection as displayed on the ILS indicator;

(iv) No unusual roughness or excessive attitude changes occur after leaving the middle marker; and

(v) In the case of an aircraft equipped with an approach coupler, the aircraft is sufficiently in trim when the approach coupler is disconnected at the decision height to allow for the continuation of a normal approach and landing. 
Given these operational requirements for an ILS approach, it can be inferred that an autopilot designed to fly such an approach should be designed to meet these parameters. For an autopilot to fly a SNI approach all of the preceding requirements apply; additionally the aircraft must navigate with accuracy enough to ensure it remains within the airspace allotted for the SNI approach. The FAA has begun to address the issue of how precisely an aircraft is capable of navigating in terms of Required Navigation Performance (RNP). Area navigation (GPS/INS) approach and departure procedures are assigned an RNP value, stating the performance that is required to fly a particular procedure. The final approach segments of most GPS approaches in service are assigned a RNP value of 0.3 nautical miles, meaning the aircraft must be able to navigate within 0.3 miles of the specified track. Presently RNP refers more to navigation signal accuracy and sensitivity than control system precision; however as approaches require tighter RNP values, ability of autoflight systems to maintain a more precise track will likely become a factor in certification of RNP performance (5).

\section{THE PolyQSRA IMPLEMENTATION}

The QSRA mathematical model was designed to be compatible with the Ames Research Center's FSAA simulation facility. It was utilized in both the design and testing processes of the aircraft, and also for pilot familiarization. The Math Model describes a full Six-Degree-of-Freedom (6DOF) simulation; all components that affect the flight characteristics of the QSRA are simulated. This includes “free-air” aerodynamics, primary control surfaces, flaps, spoilers, engines, and the BLC system. Descriptions of the stability and control augmentation systems and engine dynamics are included as well. The simulation parameters are defined by the Math Model in figures such as Figure 17. 


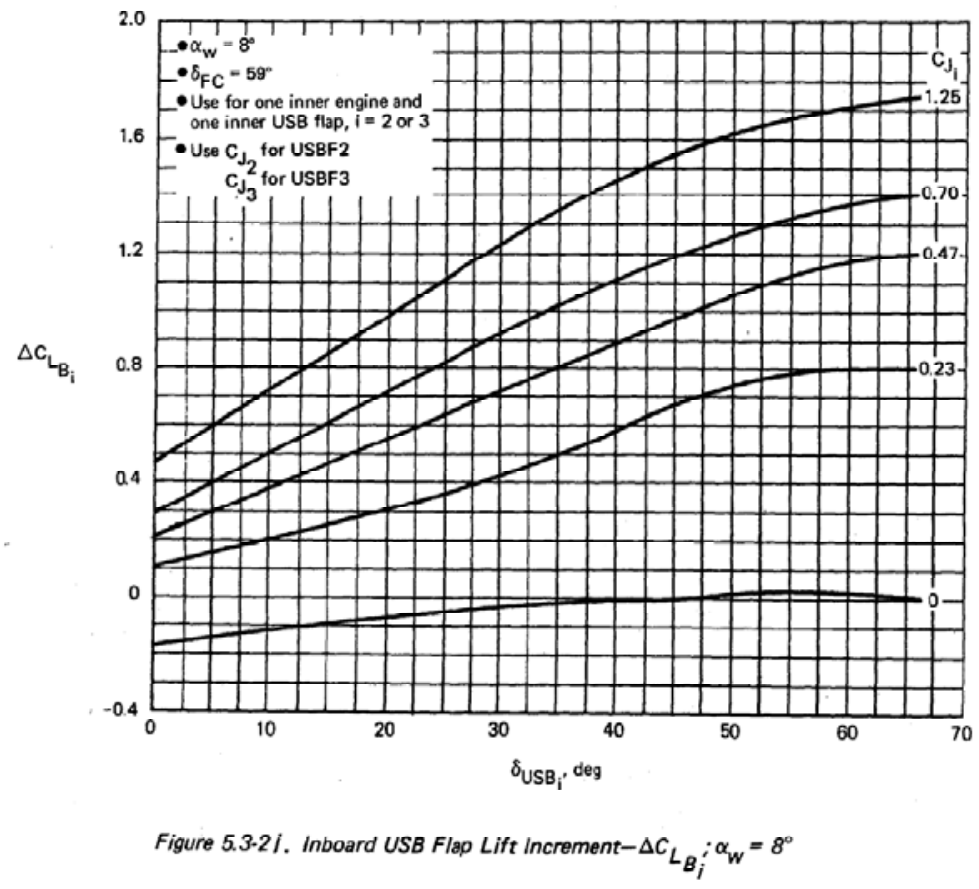

Figure 17 - Example Math Model Parameter Figure (1)

The Math Model contains approximately 200 pages of these types of figures, which were all digitized into table lookup form for use in the PolyQSRA simulation. Individual parameters are defined by tables ranging from one-dimensional to five-dimensional, meaning a single table lookup can be based on as many as five inputs. The Math Model utilizes a modular method of construction, meaning changes can be made to one module without affecting other modules. With few exceptions, all modules are re-created in their entirety in PolyQSRA. The following discussion will give a brief overview of each module and then focus on any differences between the Math Model and PolyQSRA implementations. For a more in depth description of each module, the QSRA Math Model may be referenced (1).

\section{EQUATIONS OF MOTION}

PolyQSRA utilizes an Euler angle implementation of the equations of motion. For purposes of completeness, the description of the equations of motion is included in the QSRA Math Model (1). The equations of motion are identical to those used in the NASA-AMES Flight Simulator for 
Advanced Aircraft (FSAA), and are described in A Standard Kinematic Model for Flight

Simulation at NASA-AMES (2).

The equations of motion can simulate a rotating earth, but the "flat earth" (rotational velocity of the earth equals zero) assumption is acceptable for the QSRA, as it does not fly in outer-space or particularly fast. The general flow of the equations of motion is shown in Figure 18. All of the external forces and torques are masked by a single subroutine. Aerodynamic forces are handled in the stability axes (referenced to angle-of-attack [AOA]) within the external forces and torques subroutine; output from this subroutine, however, is referenced to body axis.

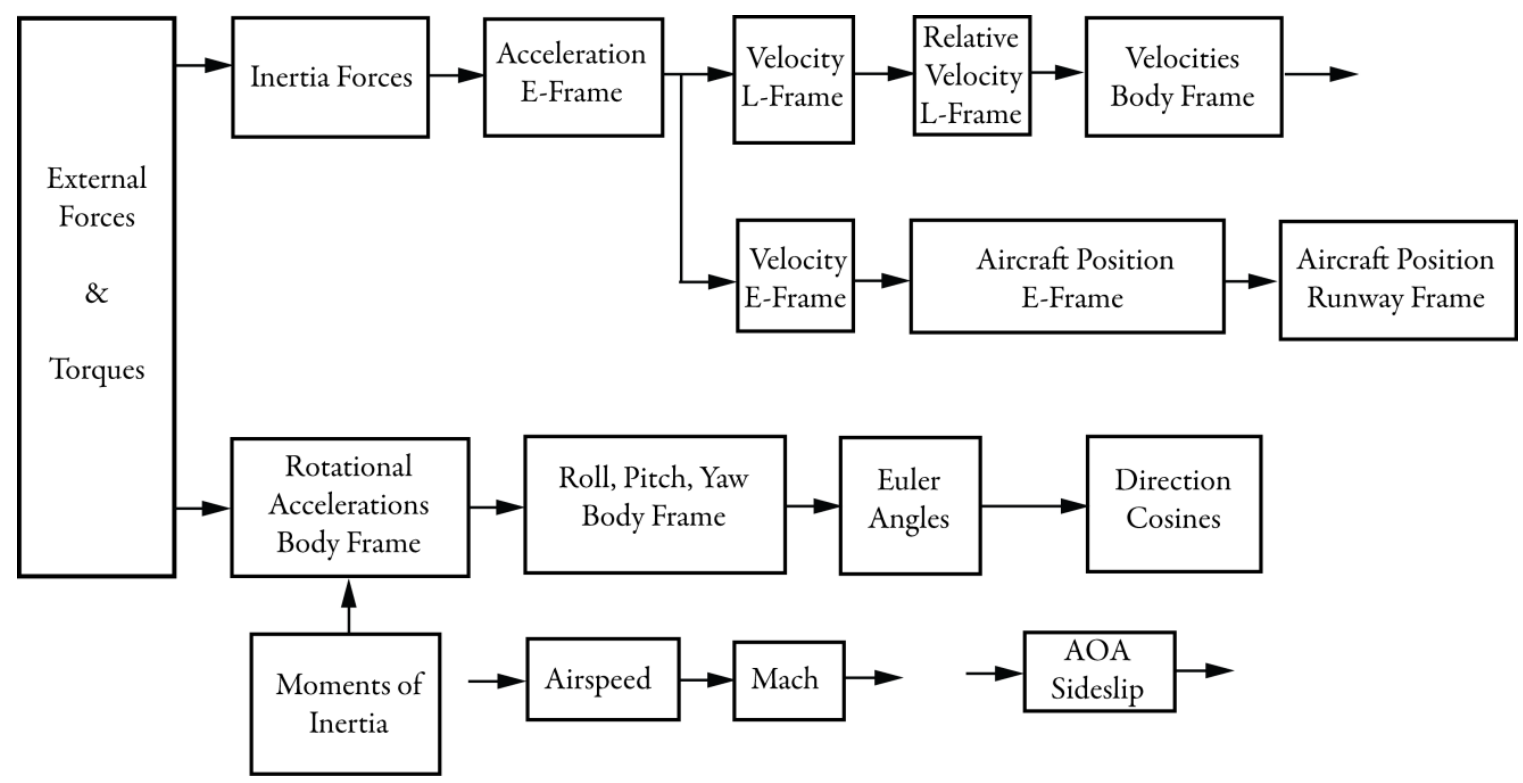

Figure 18 - Equations of Motion

The upper block flow in Figure 18 performs the transformations and integrations necessary to determine the accelerations, velocities, and positions in the earth frame. Provisions are made to add wind into the local frame, as well as to "inject" turbulence into the body frame. A translation is provided to convert the earth frame position coordinates (latitude, longitude, altitude) to runway frame (distance down runway, distance from centerline, and height). Runway frame coordinates are used by PolyQSRA for generation of navigation signals for the instrument 
approaches. For the purposes of PolyQSRA, runway frame is adequate for simulation of ground based navigational facilities as well as GPS, but it should be considered that a true navigation simulator would include additional sources of error and time delay (in the case of GPS).

The lower block flow handles the rotational accelerations, velocities, and position. The moments of inertia are determined from a figure for the QSRA based on gross weight. The body-frame moments output from external forces and moments is integrated to obtain rotational velocities, and then integrated again to obtain Euler angles. The Euler angles are used to create the local-tobody transformation matrix, which is utilized for the linear velocities calculation. Various peripheral variables, such as angle of attack and sideslip are also calculated in the equations of motion.

To allow the simulation to start from a dead stop without divide by zero errors, aerodynamics are ignored for extremely low dynamic pressure situations. Instead, the simulation is passed a dynamic pressure scaled to the engine thrust setting. Force buildup for the same low dynamic pressure situations is accomplished by only considering the thrust output of the engines. As dynamic pressure increases, the aerodynamics smoothly takeover from the direct thrust forces.

\section{PROPULSION SIMULATION}

The propulsion system is modeled exactly as described in the QSRA Math Model. Inlet and nozzle forces for each engine are simulated separately for low-speed flight (Mach $<0.3$ ). The BLC system nozzle pressures are simulated, as is the effect on engine thrust due to bleed for the BLC system. Engine acceleration and deceleration dynamics are modeled as rate-limited second order responses with the addition of response lags associated with low fan speeds. Provisions to model individual engine and BLC failures are included. 
The engine model outputs a gross thrust value for each engine $\left(T_{G}\right)$, however this value is only used in the low dynamic pressure direct thrust buildup. For normal flight, the thrust force contribution is passed to the aerodynamics module in the form of a thrust coefficient $\left(\mathrm{C}_{\mathrm{J}}\right)$. This is suitable for the QSRA due to the significant aerodynamic/propulsion coupling. BLC parameters are also passed to the aerodynamics module, where BLC forces are then enumerated. Engine inlet (ram-air) forces are passed directly from the propulsion model to the external forces and torques summer.

\section{AERODYNAMICS SIMULATION}

Once again, the aerodynamics for PolyQSRA are implemented exactly as described in the QSRA Math Model. The Math Model data is derived from QSRA wind tunnel test results with the addition of some YC-14 data used for trends. As described by the Math Model (1), the aerodynamic model includes the following:

- Six component forces and moments for the following:

o All flap settings (including flaps up)

- All control surfaces and flap surface deflections

o Powered-lift blowing effects in all axes

- Leading-edge and aileron BLC effects, including BLC failure and thrust recovery

o Ground proximity effects

o Engine-out effects

- The following limitations are applicable:

o $V_{e}<200$ knots (no Mach or aeroelastic effects included)

o Except for takeoff roll, data limited to $-8^{\circ}<\alpha_{w}<32^{\circ}$...

- Post-stall effects not fully simulated...

- Model produces match with critical performance points

The aerodynamics module handles the direct engine force buildup for low dynamic pressure simulation (i.e. a standing start takeoff), as well as the transformation of the aerodynamics data from stability axes to body axes. Aerodynamic terms are handled in stability axes. Each of the six aerodynamic force variables is built up by an equation, such as Equation 1. 


$$
\begin{gathered}
C_{L}=C_{L_{F A}}+K_{F R E F} \Delta C_{L_{U S B}}+\left(1-K_{F R E F}\right) \Delta C_{L_{U S B O}}+\Delta C_{L_{G E}}+\Delta C_{L_{\text {Tail }}} \\
+\Delta C_{L_{\text {Flap }}}+\Delta C_{L_{\text {Spoiler }}}+\Delta C_{L_{\text {Aileron }}}+\Delta C_{L_{\text {Sideslip }}} \\
+\Delta C_{L_{U S B O}}+\Delta C_{L_{L E B L C}}+\frac{\bar{C}_{W}}{2 V_{R W}^{*}}\left[C_{L_{\dot{\hat{\alpha}}} \dot{\alpha}}+C_{L_{\dot{\hat{q}}}} Q_{S}\right]
\end{gathered}
$$

Each variable in Equation 1 is typically formulated from another equation. The variables for that equation generally come from one or more of the more than 200 figures that make up the aerodynamic data. A sample aerodynamic data figure is shown in Figure 17. A single aerodynamic parameter is determined by between one and four simulation, propulsion, control surface, or aerodynamic variables.

Thrust coefficients are used in the aerodynamics figures to take into account the effects of powered lift. Thrust to propel the aircraft forward is determined from the thrust coefficients and is implemented as negative drag.

\section{FLIGHT CONTROL SYSTEM}

The purpose of the flight control system module is to provide the control surface positions to the aerodynamics module. A full description of the QSRA flight control system is provided in the Math Model (1). The Math Model attempts to replicate and describe all aspects of the flight control system, including mechanical and electrical paths, as well as the control column force loader system. This creates some issues for PolyQSRA, however, because a QSRA simulator cab with a force loader control column is not available. Instead provisions are made to "adjust” the flight controls to operate without the normal hardware. The simulation is now capable of either being hand flown by interfacing a USB joystick with Simulink or being flown completely on “autopilot” with no joystick attached. 
The biggest challenge to replicating the flight control system was emulating the column force loader system. The original elevator deflection is mechanically geared to column position, and a true QSRA column is not available. Trim in the actual aircraft is accomplished by adjusting the center position of the force loader. Pitch feel force gradients are described by Figure 19. Column feel forces are determined by airspeed and how far the elevator is deflected from its trim position. On the physical aircraft, the PCAS utilizes a load cell attached to the pilot's control column to generate PCAS pitch commands. Thus, it is necessary for PolyQSRA to "back out" the force that the column would be producing in order to generate the PCAS force command, as shown in

Figure 20.

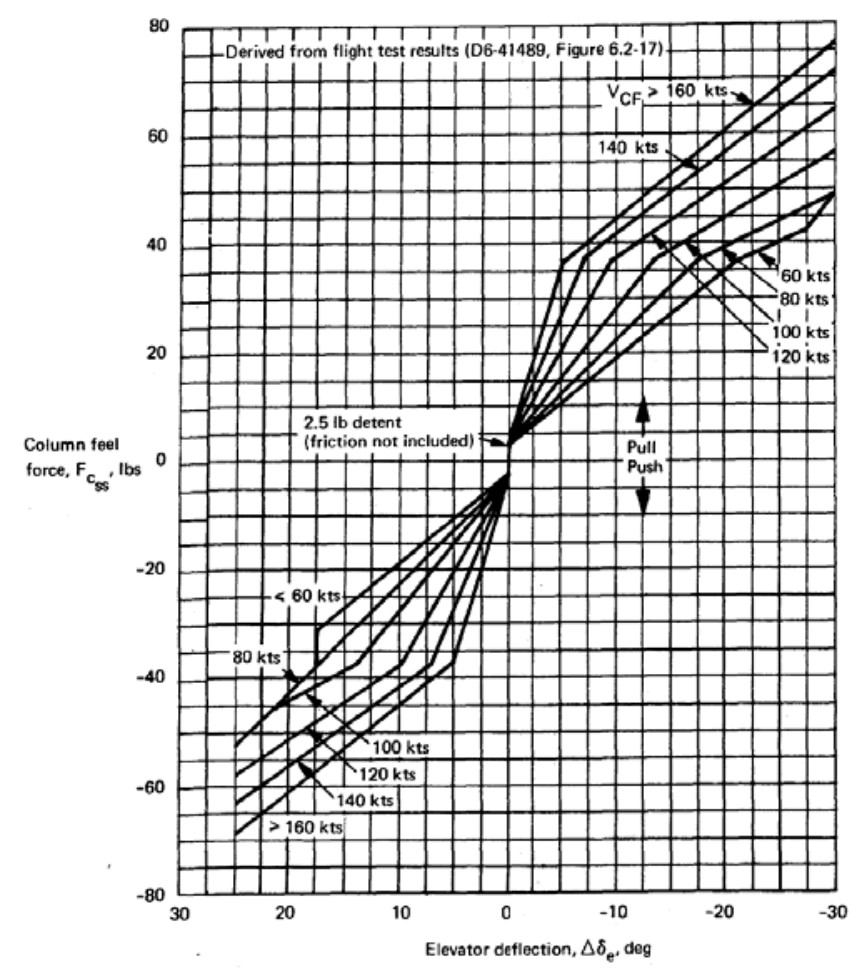

Figure 19 - Pitch Feel Computer Force Loader Gradients (1) 


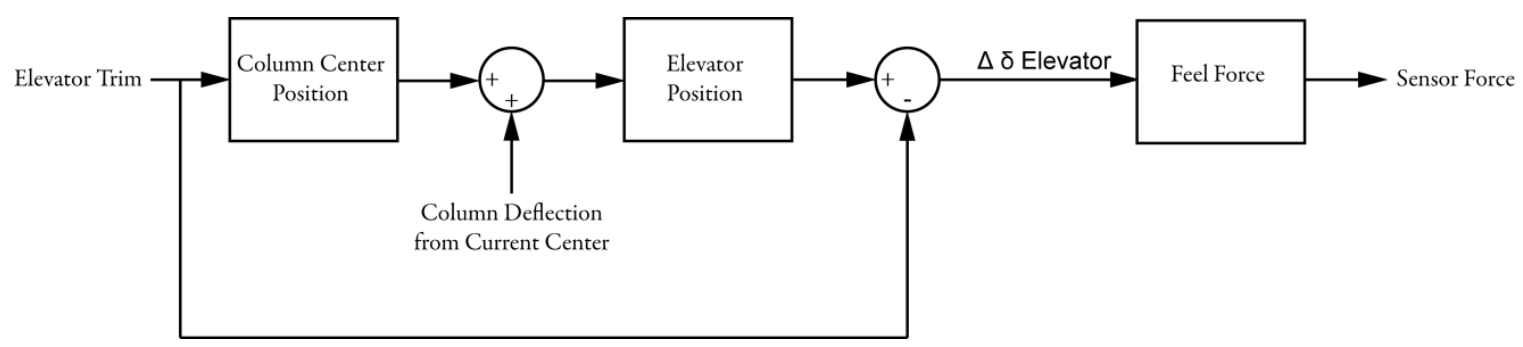

Figure 20 - PolyQSRA Column Force Workaround

This workaround results in a simulated force that can be output to the pitch control law for processing. Trim is accomplished by moving the "effective" center position of the stick; the stick center position always remains the same when using a USB joystick.

PolyQSRA evolved, however, and this force value is now ignored due to other complications (though it is still available in the model for future work). The purpose of using the load cell force in the physical aircraft is to provide a constant pitch rate per pound column force. When there is no force on the column (stick "in detent”), the PCAS reverts to attitude hold mode.

The issue with this system encountered with PolyQSRA seems to be due to lack of clarity in the Math Model as to how the force load input to the PCAS, mechanical column to elevator input, and PCAS actuator all work together. The Math Model seems to describe a system where to pitch up, the pilot pulls back on the control yoke. This deflects the elevator due to the mechanical/hydraulic control path, but it also sends a pitch-rate command to the PCAS. As the PCAS receives a pitch-rate command, the aircraft is in fact already pitching due to the elevator deflection on the mechanical path. Thus, PCAS signals provide adjustments to the already deflected elevator in order to obtain the desired pitch rate. This system resulted in unacceptable pitch transients when implemented on PolyQSRA. Probably the transients can be attributed excessive actuator lag or confusion as to how to implement the pitch system without physical QSRA components, but it is still not particularly clear how this system worked on the actual aircraft. 
The constant pitch rate per force gradient system was abandoned on PolyQSRA. Instead, a system was adopted where the PCAS provides attitude hold up until the stick moves out of detent. The PCAS then temporarily disengages; a "pull” on the column results in a pitch up dictated by the mechanical path. Then as the column is released, the PCAS reengages, smoothly building in an elevator bias in order to hold pitch attitude constant. The system then re-trims the column so that steady state elevator commands are "held" by the column position trim rather than a PCAS command. This also ensures that the aircraft will be in trim if the PCAS is disengaged. The original Math Model specifies a trim offload system: it has been given increased authority on PolyQSRA in order to function with the modified system.

Another difference with PolyQSRA is the elimination of separate flight control system actuators. The Math Model specifies two sets of actuators for each primary control surface. The primary hydraulic actuator is connected to the mechanical cables from the control column or rudder pedals. Each surface also has a second "electric signal” actuator that is utilized to introduce SAS and PCAS inputs into the system. The Math Model, however, is not particularly clear about how the electric signal actuator should be connected to the system. Connecting it in series with the primary actuator resulted in excessive actuator lag and poor pitch performance. Thus, these actuators were discarded in favor of sending the SAS and PCAS signals directly to the primary actuators. This is a reasonable modification because the SAS and PCAS actuators are not an important element of the simulation, and any newer aircraft would be controlled in this manner with the use of fly-by-wire.

With these few modifications, the flight control system operates as designed. The roll and yaw stability augmentation systems are quite effective at damping out the aircraft's Dutch-roll tendency, which is quite pronounced with the SAS turned off. The PCAS is effective at maintaining pitch attitude through the flight regime of airspeeds, power settings, and aircraft 
configurations. Slight pitch transients occur during aircraft reconfiguration or large power setting changes. The PCAS attitude hold system reduces pilot workload while hand flying, and provides the inner loop closure for PolyQSRA's pitch autopilot functions, which are described in the autopilot implementation section.

\section{LANDING GEAR MODEL}

The landing gear is modeled as specified in the QSRA Math Model. This includes an oleo model, friction model, and brake model. The landing gear on the QSRA is fixed, so its aerodynamic contribution is simply taken into account in the full aircraft aerodynamics. The landing gear specified by the Math Model is not a true engineering representation of the QSRA landing gear; rather it is modified to provide "good simulation dynamics.” The original engineering representation of the landing gear was subject to "real-time simulation anomalies" such as "square wheels" and abnormal bounce at touchdown. Thus, though PolyQSRA landing gear is a replica of the Math Model, it is not necessarily true to the actual aircraft. The aircraft/landing gear combination is capable of simulating takeoff from a standing start as well as landing through touchdown. Braking in the simulation seems probably less effective than it should be.

\section{ATMOSPHERE MODEL}

The QSRA Math Model utilizes the 1962 ARDC standard atmosphere rather than the more common 1976 International Standard Atmosphere (ISA). In the interest of ensuring congruency with the rest of the model, the 1962 ARDC model is used with PolyQSRA. The atmospheric model specifies a low altitude wind shear model which is implemented in PolyQSRA and can be turned on or off. The Math Model also specifies an implementation of the Von Karman PSD turbulence model. The model specified, however, did not seem to be entirely complete. Thus, the Von Karman turbulence model available in the Simulink Aerospace Blockset is utilized. The 
wind shear model provides variable winds to the wind inputs in the equations of motion, whereas the turbulence model directly excites the aircraft body rates in the equations of motion.

\section{INITIAL TRIM CONDITION}

It is of course desirable to be able to start PolyQSRA in airborne scenarios. This eliminates the need to takeoff and fly to an initial approach fix prior to the start of every approach. To have a successful air-start of the simulator, it is necessary to start with the engines spooled to the appropriate value for the initial flight condition, to position the aircraft at the appropriate pitch attitude, and to ensure the elevator is in trim. This will ensure any transients are avoided; such transients can cause problems that range from simple annoyance to actually crashing the simulator.

The QSRA Math Model specifies that a trim routine did exist for the model, but it was integrated with the FSAA system and is not included in the model specifications. Thus, it was necessary to develop a trim routine for PolyQSRA. A simple trim routine was inserted after the forces and moments summer and before the equations of motion.

If selected to run, the trim routine reads in the forces, but only passes the longitudinal force value to the equations of motion. Likewise, moments are read in, but not passed to the equations of motion. As the aircraft "flies" along, if the aerodynamic lift force is less than weight, a pitch-up moment is sent to the equations of motion and the situation is reevaluated. While this process continues, the autothrottles adjust the engines to obtain the desired airspeed just as they normally would, and the trim runs as necessary to balance pitching moments output from the moments summer. After some transients, values eventually converge as desired, and the trim values for throttle position, engine speed, aircraft attitude, and elevator trim are output to the workspace. 
These values are then placed into the initialization file for that particular scenario. A software switch then allows the trim routine to be bypassed since it is already known that the aircraft is starting in trim. This method is only useful for small perturbations about level flight, because it ignores the thrust contribution to lift at non-trivial angles of attack as well as the lift contribution to drag. None-the-less, it proves quite effective because it is not necessary to generate perfect trim points or to generate a lot of them.

\section{PolyQSRA AUTOPILOT IMPLEMENTATION}

The Math Model does not specify an autopilot to fly the QSRA; to the author's knowledge, the QSRA was not equipped with an automatic flight system. This provided the opportunity to design an autopilot from the ground up. A full featured autoflight system was designed for PolyQSRA with the following functions:

- Heading select

- Navigation tracking

- Altitude hold, climb/descent rate select, and altitude preselect

- Glidepath coupling

- GPS steering

- Autothrottle

The primary autopilot functions are capable of controlling the aircraft in all normal conditions and aircraft configurations, including low speed powered lift. The occurrence of "minor" transients increases as airspeed is decreased and powered lift activated by extending the USB flaps due to the coupling between power and pitch with the use of powered lift. The glidepath coupler functions well for approaches in the CTOL configuration at 120 knots, but due to the powered lift coupling acting across multiple loop closures, glidepath performance is less acceptable at STOL landing speeds. This is an area where further work could investigate the use of adaptive controllers to improve STOL glidepath performance; however, the goals of this 
project are to test approaches using today's standard autopilot technology and determine what new technology is required to make STOL SNI approaches a reality. The following details the implementation and functionality of each of the autopilot modes.

\section{HEADING SELECT}

One of the most basic autopilot modes on PolyQSRA is heading select. The pilot selects a magnetic heading, and the aircraft turns to and maintains that heading. The heading select loop is closed around the roll/yaw SAS, so only the ailerons need to be manipulated for a coordinated turn. The implementation of the heading select autopilot is shown in Figure 21.

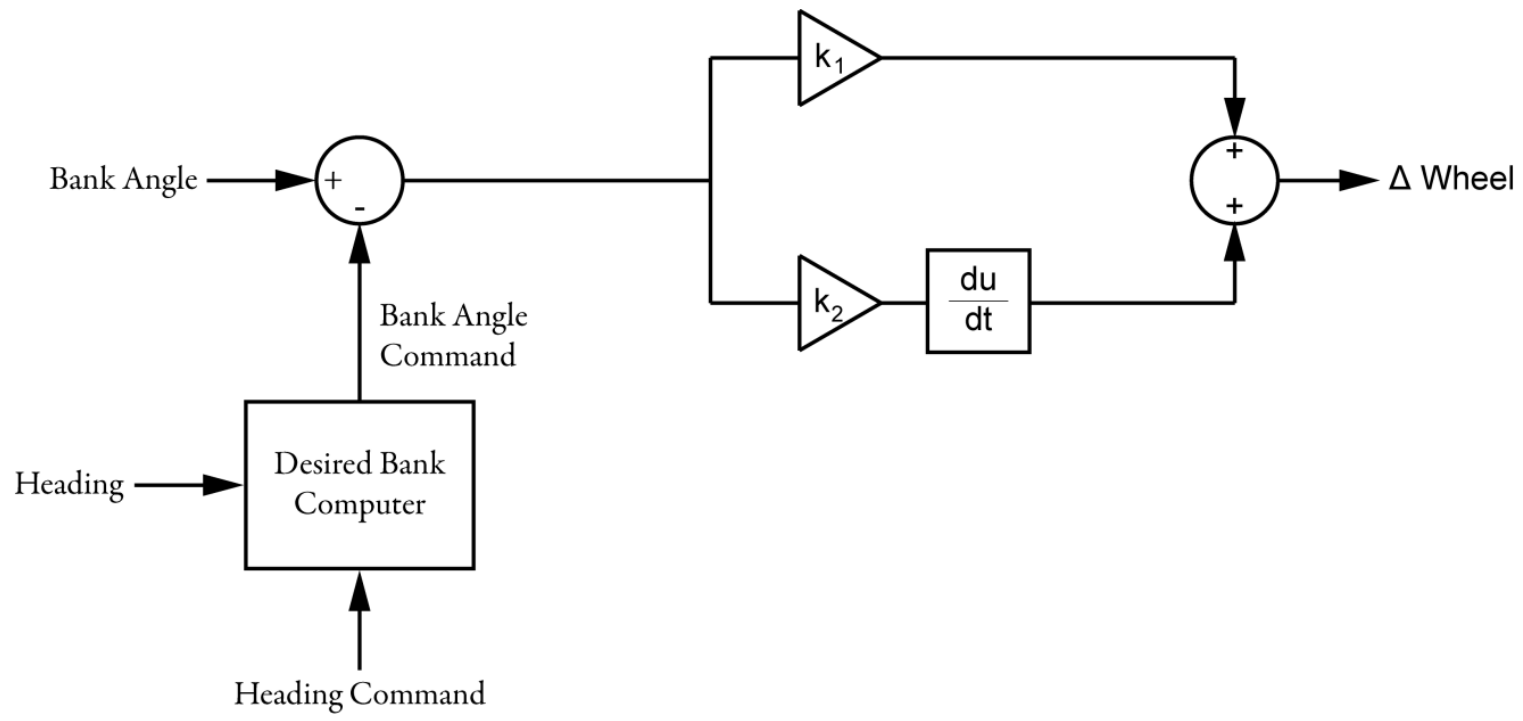

Figure 21 - Heading Select Autopilot

The autopilot heading command can come from either the pilot's input into the autopilot, or from the GPS steering module, which is explained in a later section. This heading command is then compared with the psi Euler angle (with a correction for local magnetic variation) to determine the direction and amount of turn required to reach the selected heading. For turns of greater than $20^{\circ}$, a $30^{\circ}$ bank angle is commanded. For turns of less than $20^{\circ}$, or as the desired heading is reached, the commanded bank angle is reduced proportionally to zero based on the difference between commanded and actual heading. 
The rate of change of the commanded bank angle is limited to $5 \%$ sec to ensure smooth roll-ins; roll outs are automatically smoothed as bank angle is reduced within $20^{\circ}$ of the desired heading. The bank angle command is compared with the phi Euler angle to generate the bank angle error signal. The bank angle error signal is then passed through a Proportional/Derivative (PD) controller to generate the wheel deflection command. The proportional gain commands a wheel deflection in the direction necessary to reduce the bank angle error, and the derivative gain helps prevent the bank angle command from overshooting. Aircraft response to a $90^{\circ}$ heading change at 120 knots is shown in Figure 22.

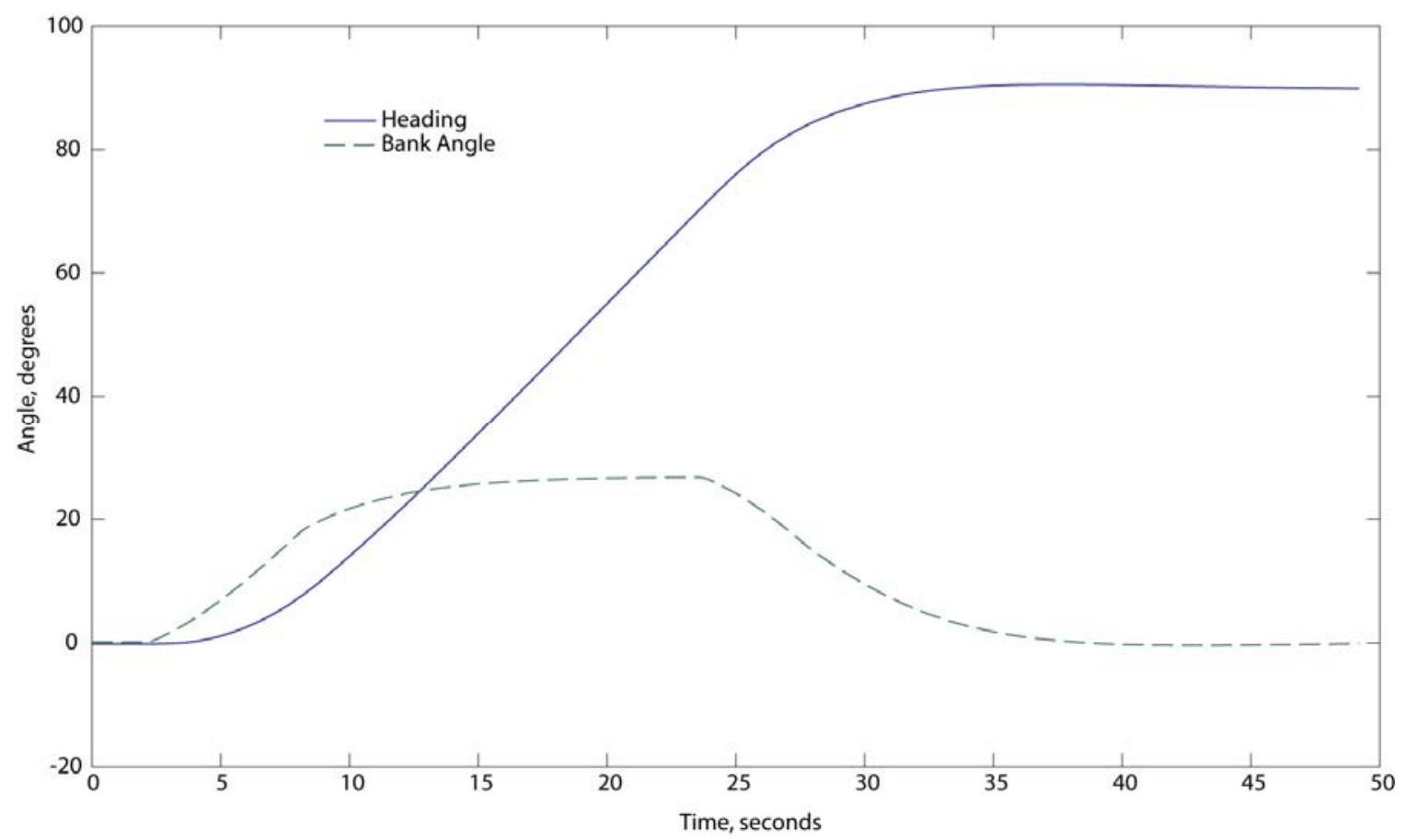

Figure 22 - Heading Change Response

\section{NAVIGATION TRACKING}

The navigation tracking mode of the PolyQSRA is designed to track simulated VOR radials and the localizer component of an ILS. The navigation tracker is also capable of tracking a simulated GPS signal, though this is not its design point. The navigation tracker obtains its navigation data from a Course Deviation Indicator (Figure 16) and is an implementation of a Type-A autopilot as 
previously described. The control diagram of the navigation tracking module is shown in Figure 23. The navigation mode requires the pilot to select the nominal tracking heading for the VOR or localizer; the controller then applies a correction to this heading based on the CDI, and then outputs the heading to be flown to the heading select module.

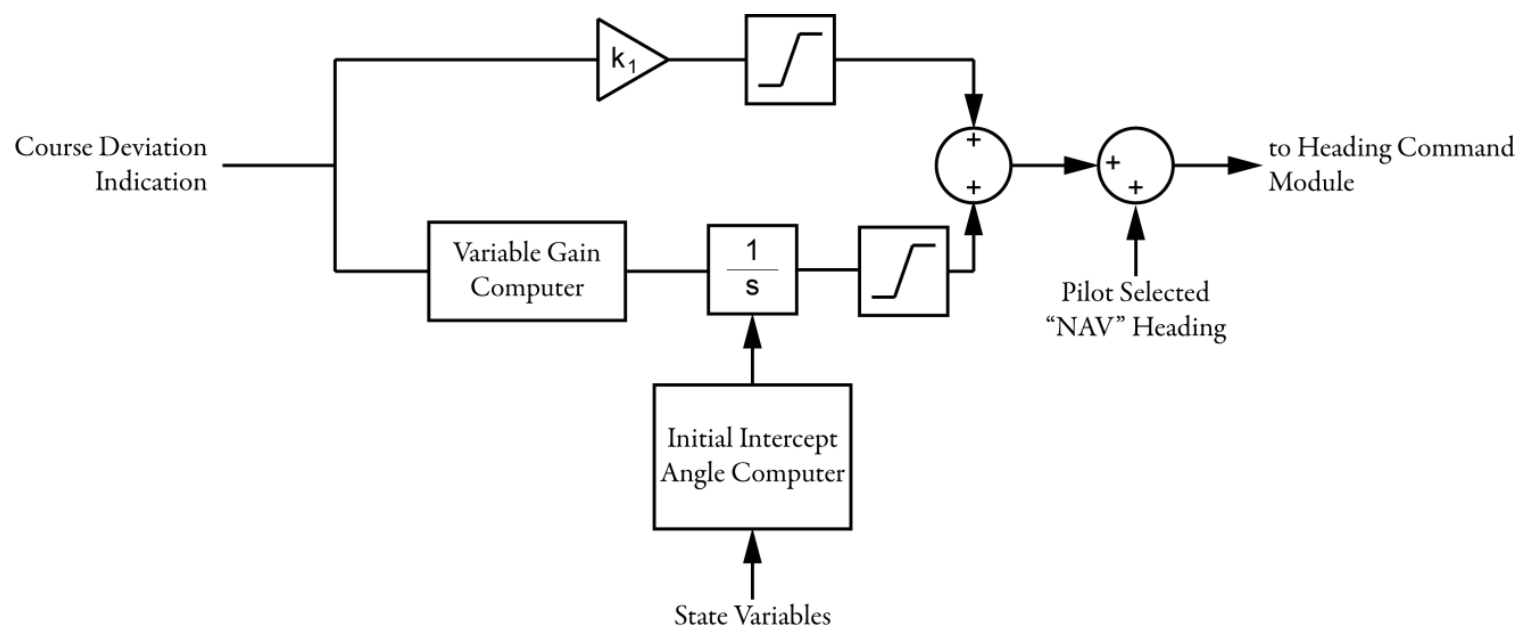

Figure 23 - Navigation Tracking Autopilot

The pilot selects either localizer or navigation (VOR) tracking; selection of localizer tracking results in a decrease of sensitivity by one half in order to compensate for the increased sensitivity of the localizer signal versus a VOR signal (approximately $4^{\circ}$ for full-scale CDI deflection vs. $12^{\circ}$ ). The CDI is already an error signal, so it is sent directly to a Proportional/Integral (PI) controller. The proportional gain is set to command a $45^{\circ}$ intercept angle from full deflection, while the integral gain is designed to add in any steady state wind correction necessary for tracking.

To prevent from loading the integrator prematurely during intercept, integral gain is scheduled based on CDI deflection. Integral gain is maximum when the CDI is centered, and linearly dropsoff to zero when the CDI is fully deflected. The integrator initial condition is set so that if 
navigation mode is selected with the aircraft established on an intercept angle less than a $45^{\circ}$, the navigation tracker will maintain the lesser intercept angle, at least until the CDI becomes active.

The navigation tracking module can be used to track a GPS signal. This is accomplished by providing distance left/right of course to the CDI, and continually updating the nominal tracking heading, which simulates the pilot turning the CDI needle as the tracking heading changes. As will be shown in the results, this method of tracking a GPS signal is not particularly accurate for curved paths.

\section{GPS STEERING}

The availability of a GPS signal opens up many new possibilities for course tracking. With GPS, it is possible to know exact ground track and speed, distance course deviation rather than degrees, and where the aircraft should be in the future. With this new information, it is possible to steer the aircraft predicatively rather than reactively.

The GPS steering module of PolyQSRA bypasses the navigation tracking module and sends a heading to fly directly to the heading command module. It generates a heading command based on pointing the aircraft directly at where it should be at some fixed time in the future. This results in a feed-forward type system, which is more suitable for tracking a curved path than a feedback system. Figure 24 depicts this implementation of GPS steering. 


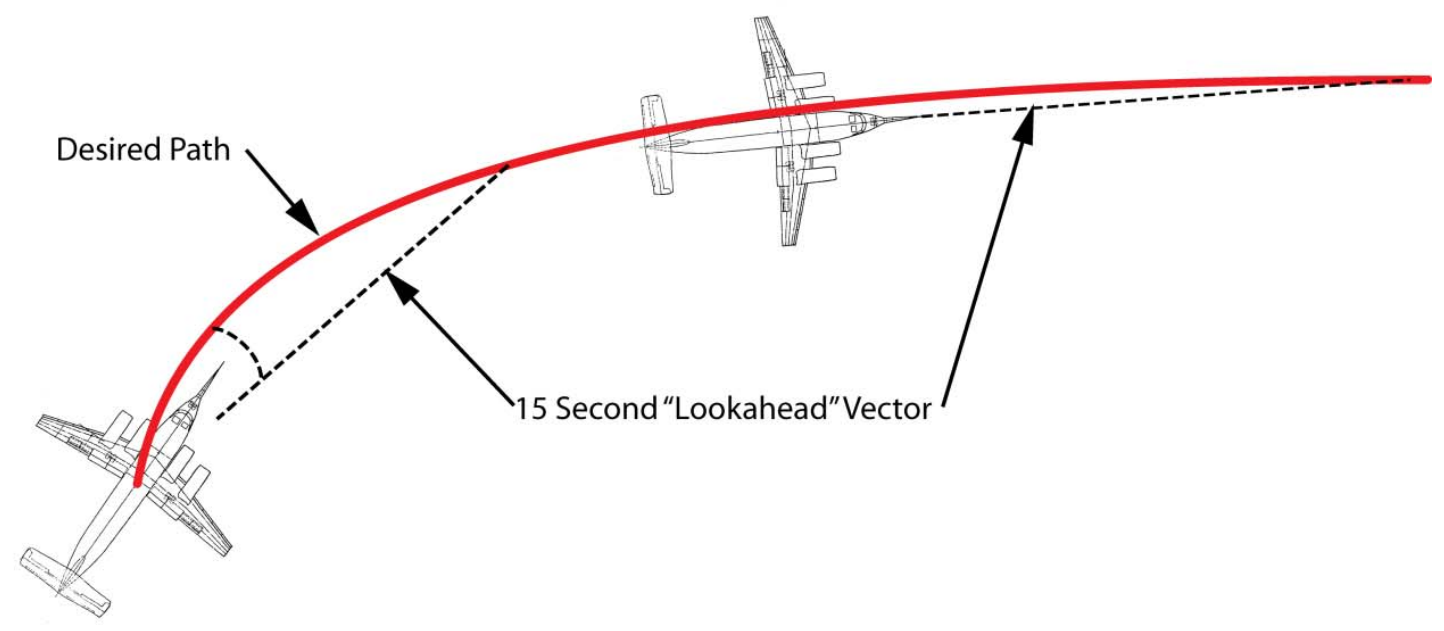

Figure 24 - GPS Steering Example

This implementation of GPS steering will always result in the aircraft tracking to the inside of an arced segment; thus a small turn bias in the direction of the course deviation has been included. GPS steering should be all but immune to wind effects because the heading commanded to the aircraft already includes a correction for the ground track based on GPS data. The logic for the GPS steering is included in Appendix III.

\section{ALTITUDE HOLD/RATE COMMAND}

The pitch autopilot for PolyQSRA maintains a specified rate of climb or descent, with altitude preselect and altitude hold. The autopilot maintains the specified rate to within 500 feet of the preselected altitude. Within 500 feet of the selected altitude, the commanded rate is reduced linearly in a manner so that at 100 feet from the selected altitude, the commanded rate is 500 feet per minute. Within 100 feet of the selected altitude, the commanded rate is reduced linearly to zero. Finally, within five feet of the selected altitude, the commanded rate is equal to five times the altitude error in feet. It is not necessary to “arm” altitude capture with PolyQSRA; the aircraft will always level off at the selected altitude. If a level off is not desired, the preselect altitude can 
simply be set to an altitude that the aircraft will not reach (for example, in the opposite direction of the climb or descent. The control diagram for the pitch autopilot is shown in Figure 25.

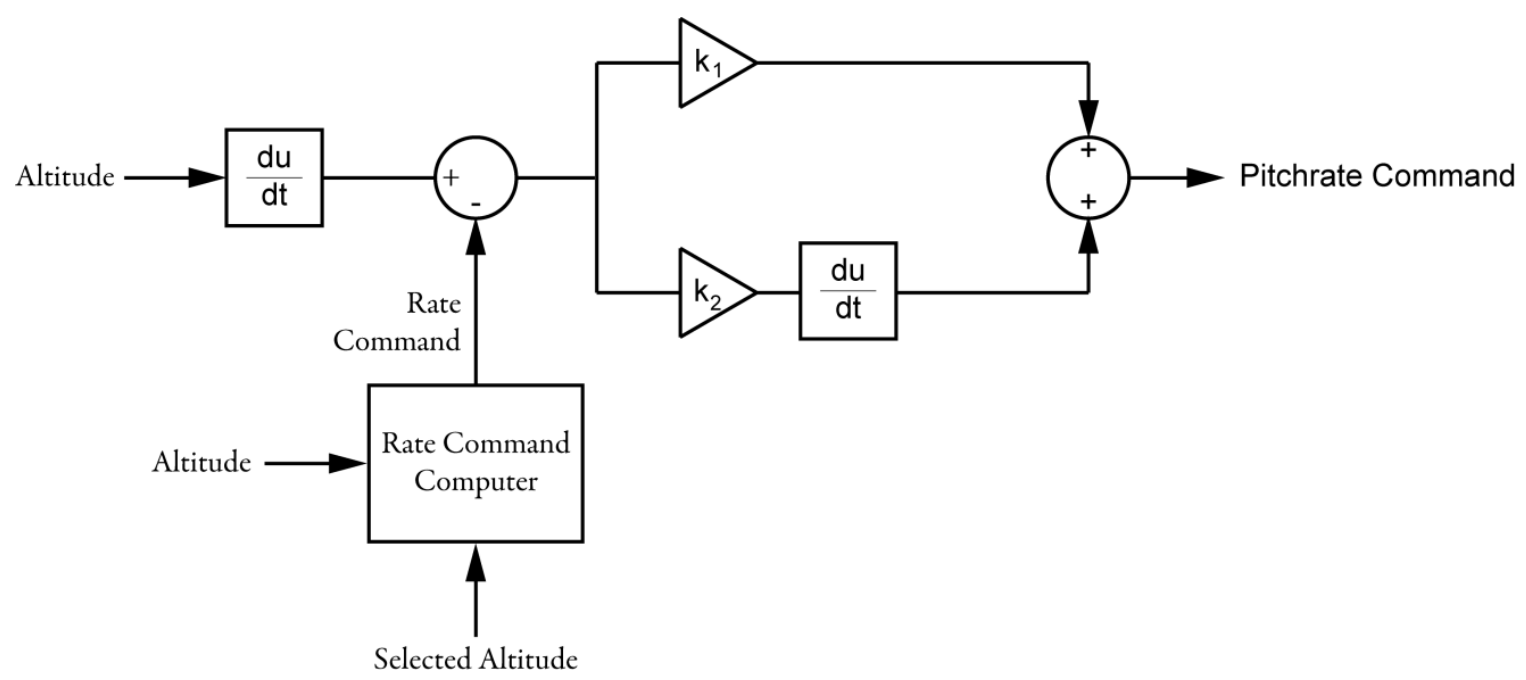

Figure 25 - Pitch Autopilot Control Diagram

The rate/altitude hold autopilot creates an outer-loop closure around the attitude hold PCAS (previously described). The error signal is generated by taking the derivative of the instantaneous altitude (assumed to be available with INS), and comparing the actual aircraft climb/descent rate to the rate commanded by the logic unit, using the logic as specified above. PD compensation is utilized to generate a pitch rate command, which is sent directly to the attitude hold control system. It was determined that this method of exciting the pitch axis of the PCAS was desirable over simulating moving the control column.

Autopilot response to an altitude change command from 2,000 feet to 4,000 feet at a climb rate of 2,000 feet per minute with autothrottles set to 120 knots is shown in Figure 26. Figure 27 depicts the performance of the altitude hold mode in "moderate” turbulence at 120 knots. 


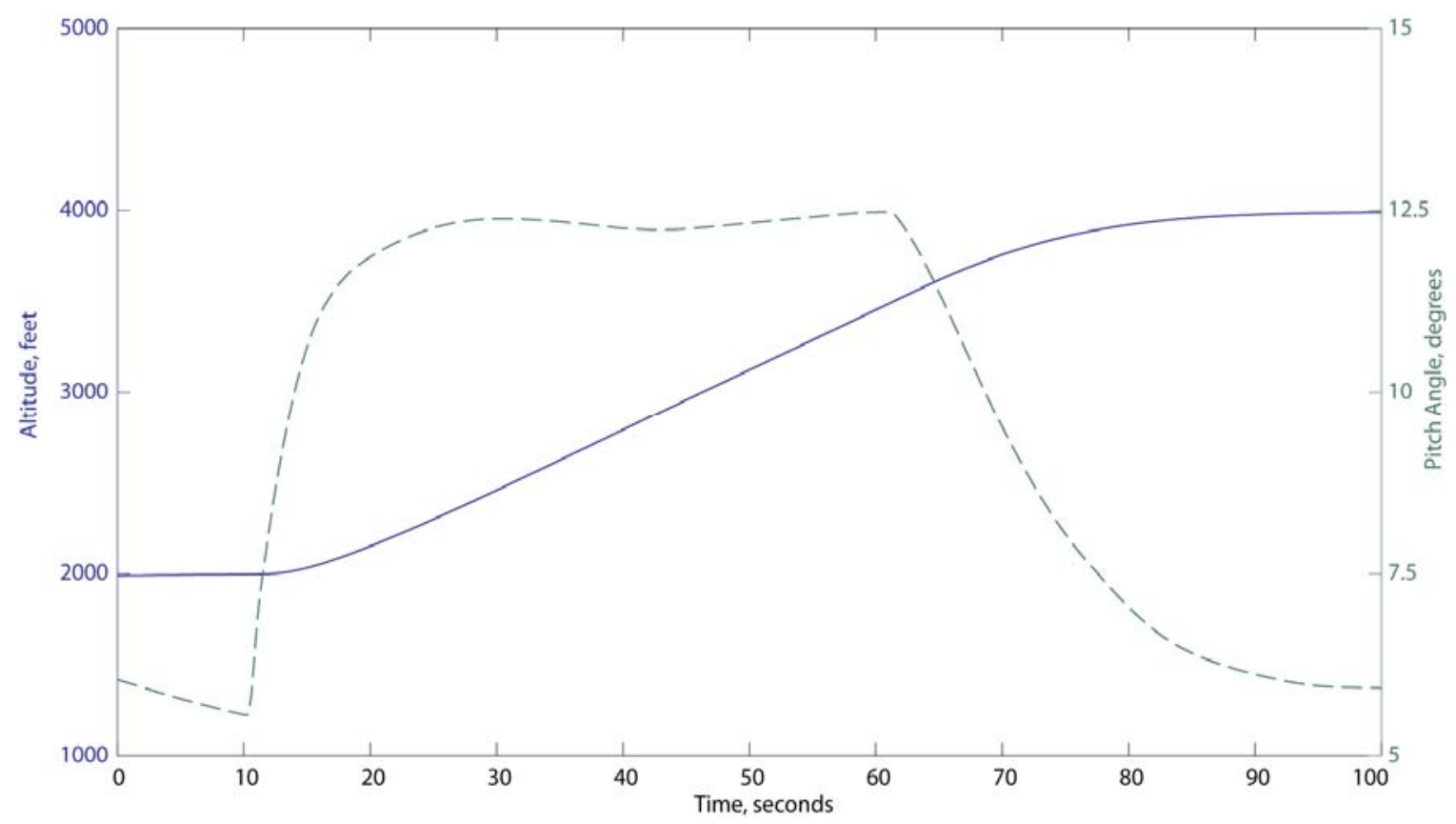

Figure 26 - Autopilot Altitude Change Response

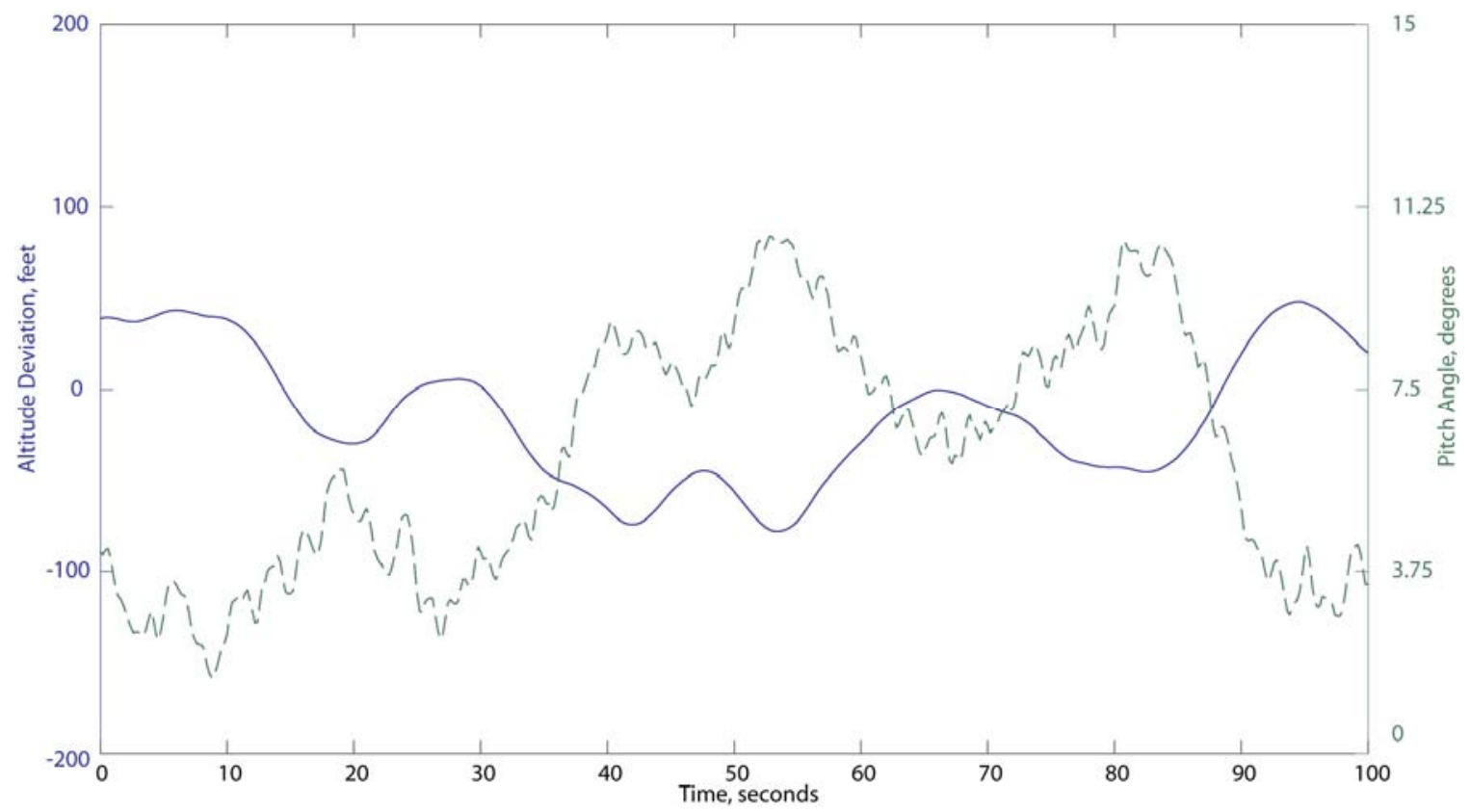

Figure 27 - Altitude Hold in Moderate Turbulence

\section{GLIDESLOPE COUPLER}

The glideslope coupler proved to be the most challenging autopilot element in terms of achieving acceptable performance. In keeping with the theme of using current generation technology, the glideslope coupler utilizes a PID controller. The glidepath coupler, however, incorporates several 
modifications that do not require the observation of any additional states. Additionally, the PID gains for the glidepath coupler are left as variables that can be set by an initialization file to facilitate trying different combinations of gains when tuning performance. The control diagram for the glidepath coupler is shown in Figure 28.

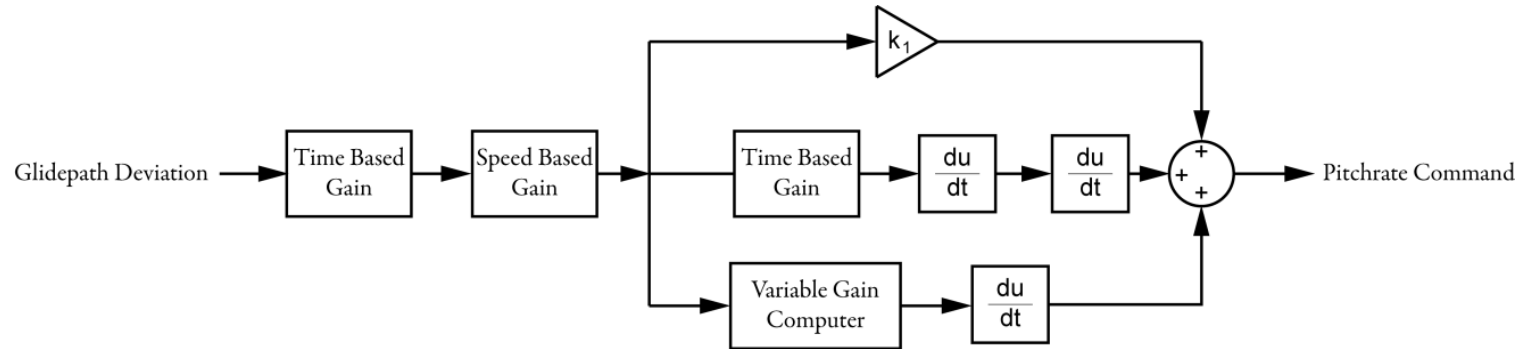

Figure 28 - Glideslope Coupler

Like the rate/altitude hold module, the glidepath coupler closes an outer loop around the attitude hold PCAS; the HSI glidepath deflection provides the error signal. Pitch rate command is the output from the PID, so the PID is actually constructed by using glidepath error and its first and second derivatives. The actual glidepath error forms the "integral” portion of the PID.

One of the greatest difficulties in designing a glidepath coupler is balancing initial intercept performance with performance near the runway. The glidepath signal is broadcast from a point source at the runway, meaning sensitivity at the end of the approach is significantly greater than at the beginning of the approach. The gains necessary to provide acceptable intercept performance sometimes result in oscillations as the aircraft approaches the runway.

To address this issue, glidepath gains are reduced based on the time from initial intercept. Glidepath mode is armed from below the glideslope with altitude hold engaged. Glidepath coupling will not begin until the glidepath indicator moves through center, at which point the glidepath coupler takes over from altitude hold mode. Upon engagement, the entire system gain is held constant for five seconds, then ramped down to a lower, selected value over the course of 10 
seconds. This allows the glidepath capture to occur with one set of gains while tracking occurs with another set.

It was observed that it is desirable for the derivative of glideslope error gain to be more active when the error was near zero. Thus, a provision is included to scale the derivative gain based on error magnitude. This reduction of gain at the glidepath limits helps to reduce oscillatory behavior due to out of phase commands.

The second derivative of the glidepath error is especially prone to increased sensitivity as the aircraft approaches the runway. Thus, second gain based on time from intercept is implemented. Second derivative gain remains constant for the first 50 seconds from intercept, then is linearly reduced to its final value 150 seconds after intercept. Because the aircraft will always intercept the glidepath more than five miles from the runway, these values ensure that the sensitivity reduction is completed by the time the aircraft reaches the runway (assuming an approach speed of 120 knots). The rates of change of attitude command from both the derivative and double derivative paths are limited to provide some rudimentary noise filtering.

It was never the intent to implement full autoland for PolyQSRA, though a simple flare coupler is included for sake of completeness. The QSRA is designed to be landed with a sink rate of 300 $\mathrm{ft} / \mathrm{sec}$ in the conventional landing configuration or $600 \mathrm{ft} / \mathrm{sec}$ in the STOL configuration. Thus, the flare coupler simply commands a reduction in sink rate to the specified level as the aircraft descends below $50 \mathrm{ft}$ AGL. This is a departure from a more common "elliptical” flare coupler. To prevent oscillatory behavior before the flare coupler engages, the second derivative gain is zeroed at 200 feet AGL, and the first derivative gain is zeroed at 100 feet AGL (it is assumed that radar altitude is available to the autopilot). 


\section{AUTOTHROTTLE}

The autothrottle system is an integral part of the pitch modes of the autopilot. The autothrottle is designed to manipulate the throttle levers as necessary to obtain and maintain a pilot specified airspeed. Autothrottle is useful and even necessary in order to keep the aircraft on speed target while flying test cases with PolyQSRA. Like most autothrottle systems, the PolyQSRA autothrottle functions independently of the autopilot pitch functions, though the ability to add a bias to throttle command based on pitch rate is included. The autothrottle control diagram is depicted in Figure 29.

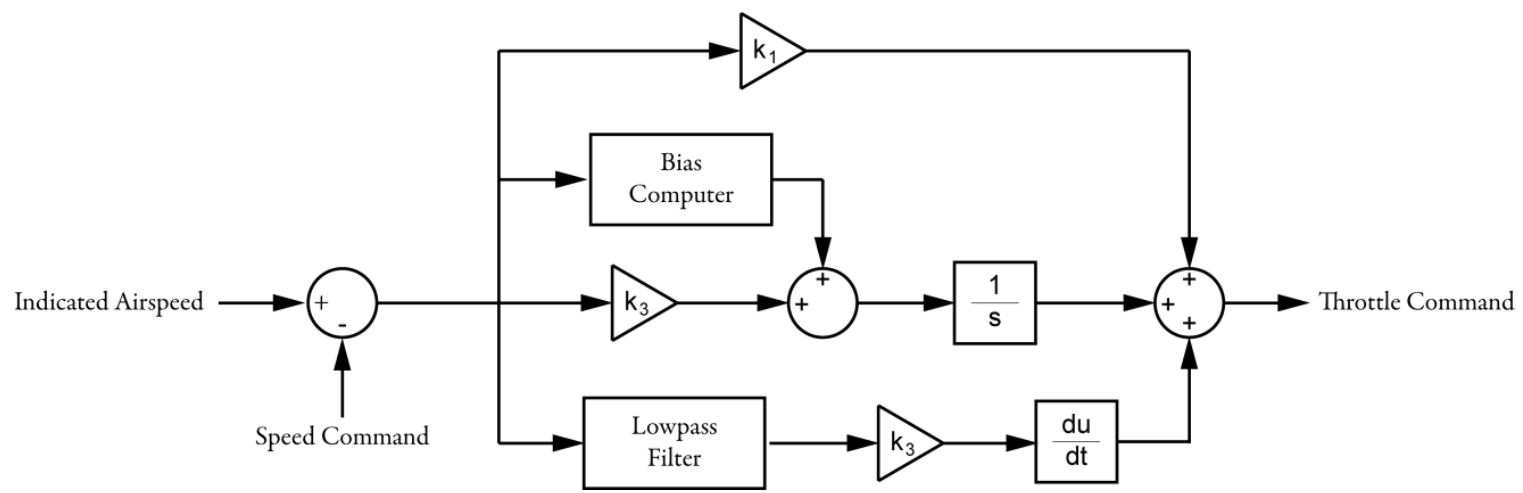

Figure 29 - Autothrottle Control Diagram

The autothrottle commanded speed is pilot selected, and is compared to calibrated airspeed in knots to generate the error signal. Calibrated airspeed is run through a low-pass filter in order to remove the effects of turbulence on the speed error. The error signal is then passed through a PID with some modifications. Modifications to the PID are handled by adding biases to the integral path. These biases are summed along with the standard integral path. As previously mentioned, one bias is included to increase throttles as the aircraft pitches up and reduce them as the aircraft pitches down, thus reducing the need for the autothrottles to "play catch-up" when aircraft attitude changes. 
The second bias is designed to improve capture response during airspeed changes. On-design performance requirements for the autothrottles dictate high gains throughout the PID, which can result in the integrator becoming "loaded" with a speed reduction or increase command. This would mean that the autothrottle would almost surely overshoot a newly commanded speed, especially considering engine spool-up times. This bias becomes active when the autothrottle is within 15 knots of the desired speed and approaching the desired speed rapidly. The bias provides a command to "unload" the integrator, providing a lead time for engine spool up/down and thus improving airspeed capture response.

Figure 30 depicts the response of the autothrottle to a commanded change of speed from 150 to 120 knots in level flight. Autothrottle response in a climb from 2,000 ft to 4,000 ft at 2,000 ft per minute with a selected nominal airspeed of 120 knots is depicted in Figure 31. Response of the autothrottles in turbulence is depicted in Figure 32.

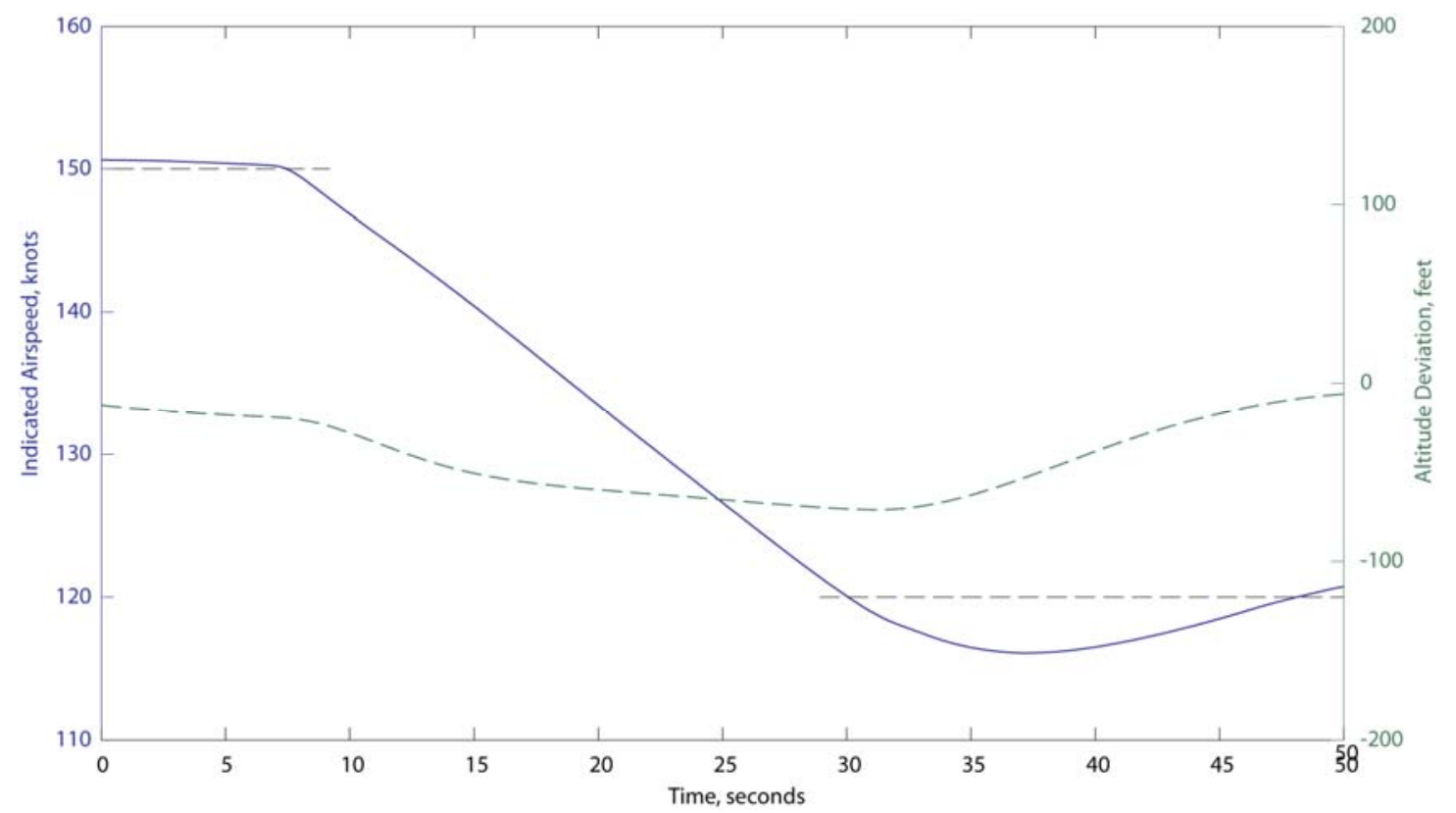

Figure 30 - Autothrottle Speed Change Response 


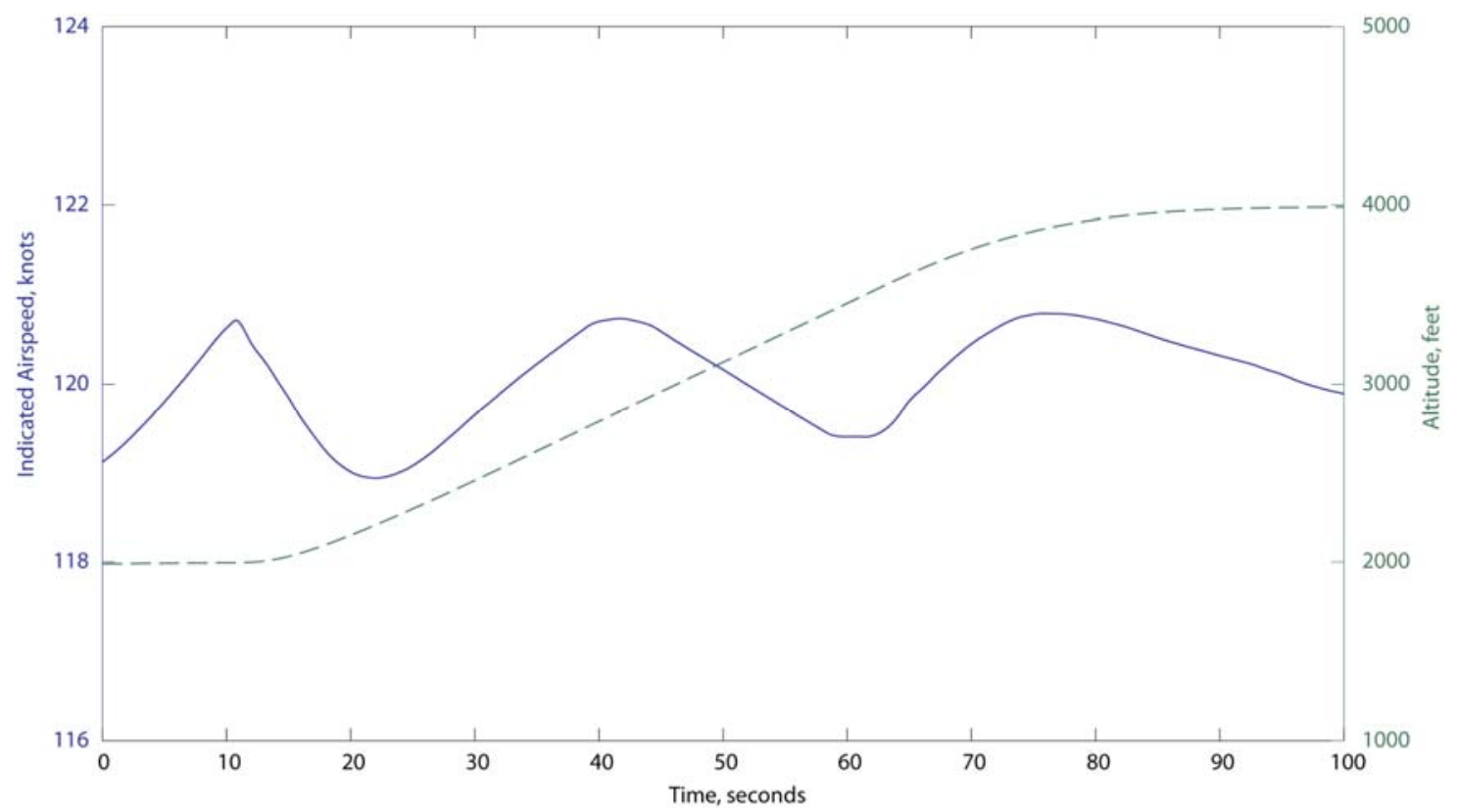

Figure 31 - Autothrottles Altitude Change Response

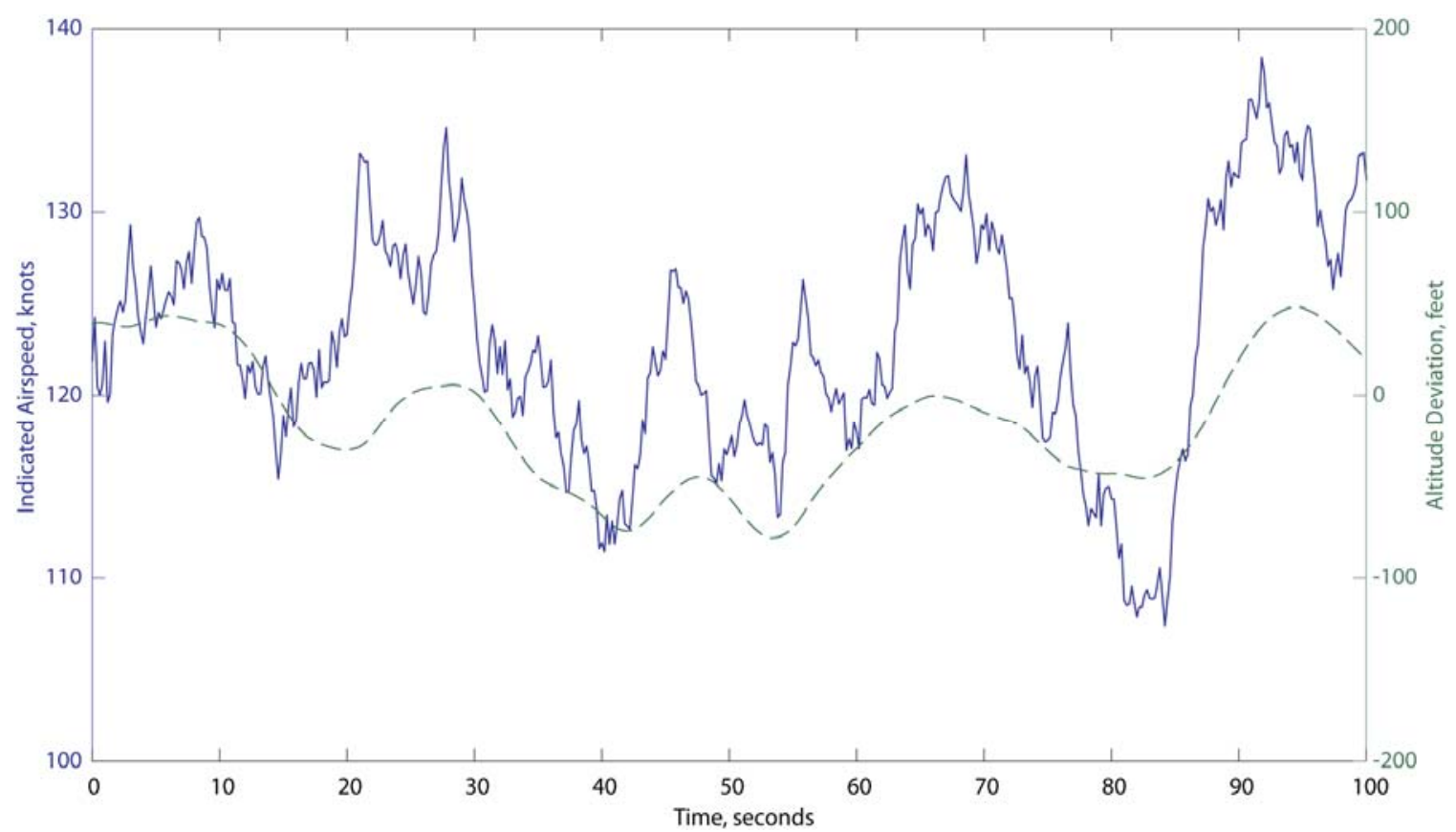

Figure 32 - Autothrottle Turbulence Response

\section{LOW SPEED AUTOPILOT PERFORMANCE}

To this point, autopilot performance has been depicted only in the CTOL configuration at an airspeed of 120 knots. The autopilot is capable of handling flight in the STOL configuration (USB flaps extended) as well, though increased coupling between pitch and power begins to 
become apparent. Additionally, little margin exists for speed/pitch excursions in the STOL configuration, and transition into and out of the STOL configuration requires careful orchestration of speed reduction and extension of USB flaps to prevent a departure from controlled flight.

Glidepath performance in the STOL configuration leaves something to be desired (as will be shown in the results section), but other autopilot performance in the STOL configuration is included as a demonstration of the autopilots abilities and robustness. Transition from 120 knots to the 85 knot STOL configuration and back into level flight are depicted in Figure 33. A descent from 4,000 ft to $2,000 \mathrm{ft}$ at 1,000 ft per minute starting from level flight at 85 knots and USB flaps deflected $30^{\circ}$ is depicted in Figure 34.

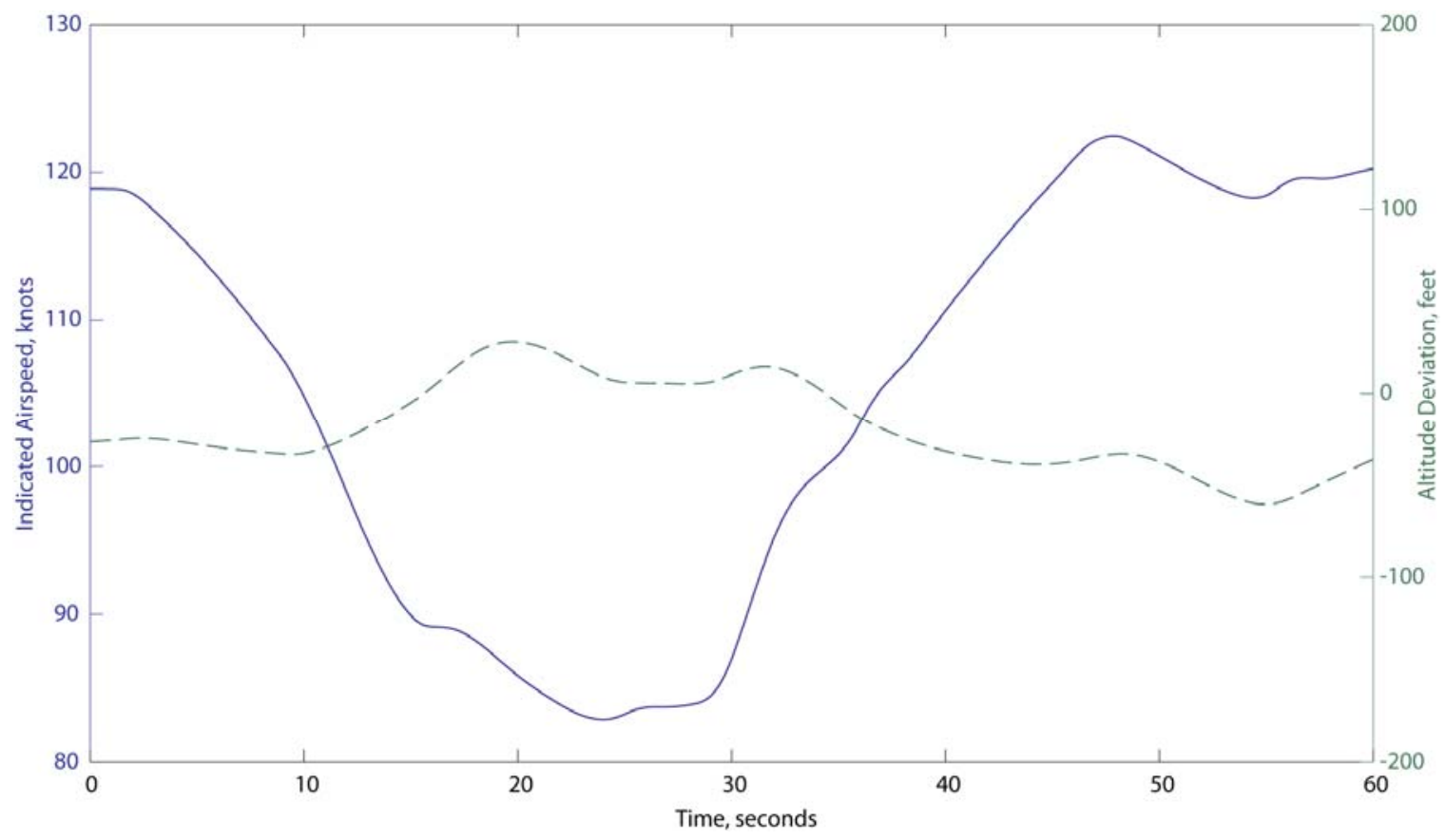

Figure 33 - STOL Configuration Transitions 


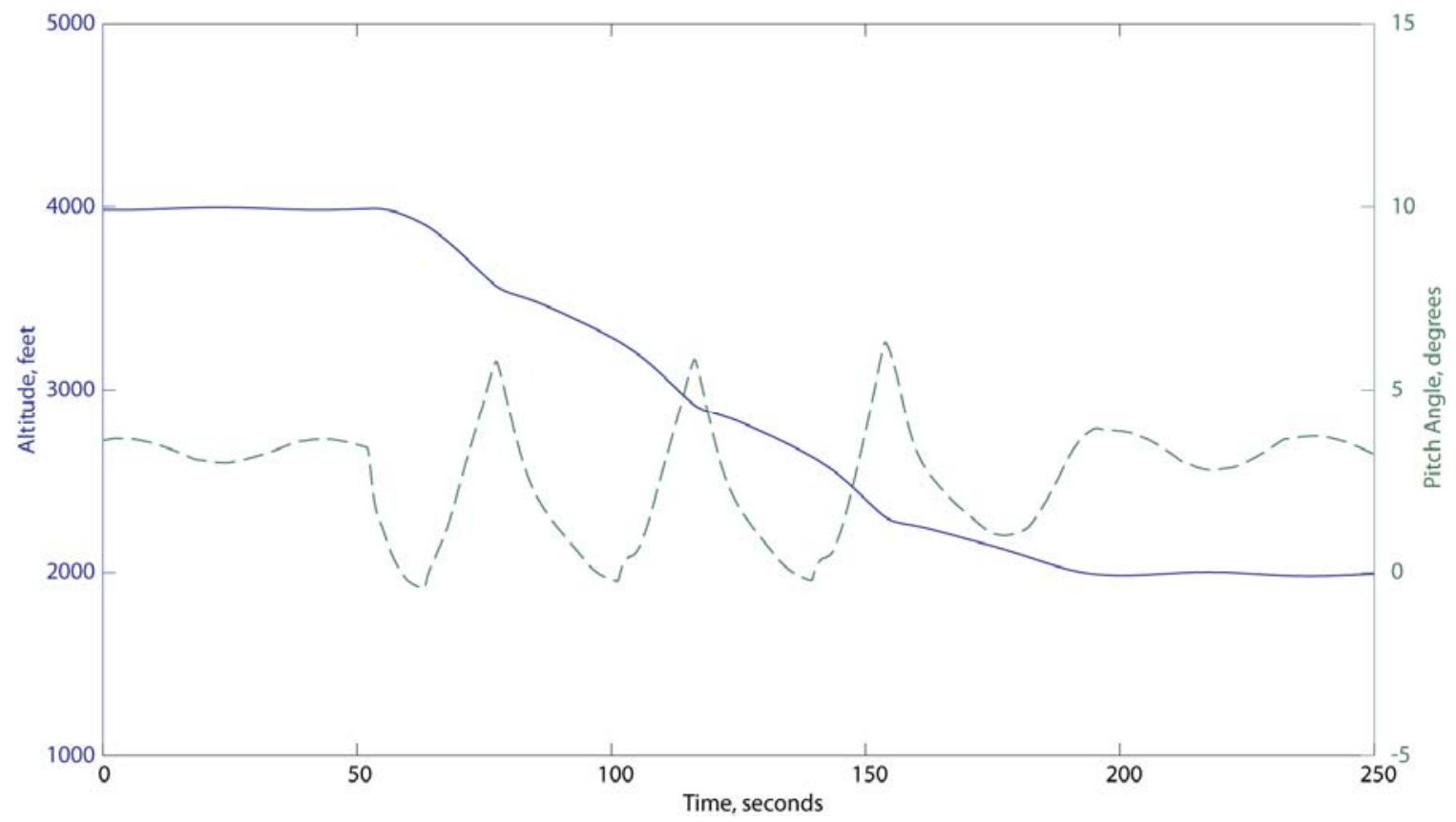

Figure 34 - Descent in the STOL Configuration

Notice the average pitch angle in the descent appears to be nearly identical to the pitch angle in level flight, indicating that pitch alone is not a good control variable for altitude in the STOL configuration.

\section{SUPPORT MODULES}

\section{VISUALIZATION}

A visual display system has not been the focus of the author's efforts. Though the simulator can be flown by a human pilot, all the testing for this investigation was done with utilization of autopilot, so real-time visualization is not specifically necessary. It has still proven useful, however, to visually depict flights. A real time visualization system is useful to put all of the data from the simulation into one easily decipherable view as the simulation is run. It can be useful to determine exactly what the simulator is doing at any point in time, as well as to identify any unusual transients present in the autopilot. Finally, it can produce nice videos for playback to demonstrate simulator performance. 
For visualization, the open source flight simulation program FlightGear is utilized (10). The Simulink Aerospace Blockset includes a module that will output real-time state variables to FlightGear. This made FlightGear visualizations an easy choice because no backend hacking was required to adapt the program to accept output from Simulink. Scenery for the entire world is freely downloadable for use with FlightGear, as are many aircraft models. Of course, there is no QSRA model available for FlightGear. As graphic realism is not a major concern, the visual model for the Beechcraft 1900 that is included with FlightGear is utilized by the author because its cockpit instrumentation is representative of a commuter class airliner. The in-cockpit view provided by FlightGear while on the final portion of a SNI approach is shown in Figure 35.

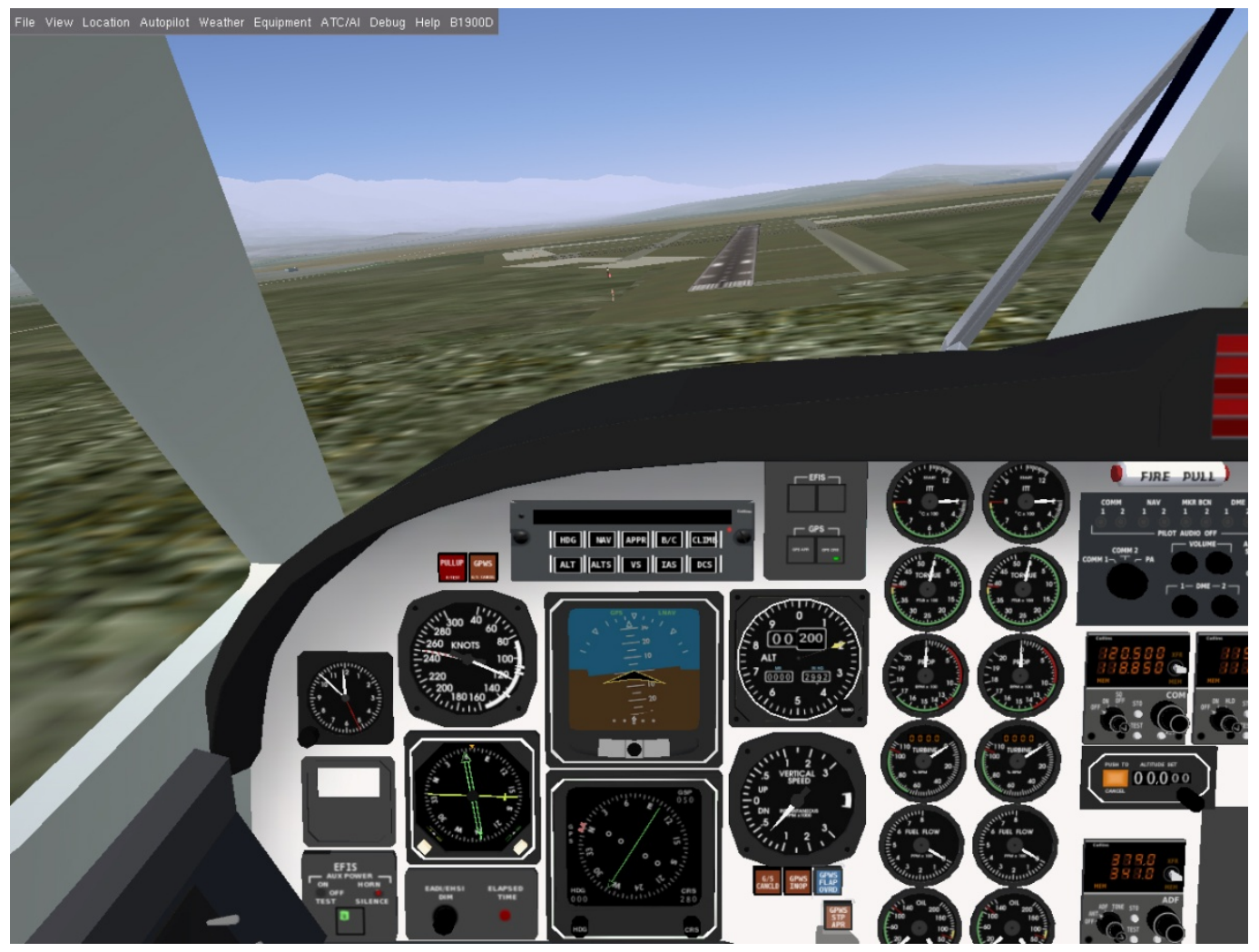

Figure 35 - FlightGear Visual Depiction 


\section{DATA AQUISITION}

The use of Matlab Simulink makes data acquisition quite simple. Any variable utilized or otherwise defined in the simulation can be recorded to the Matlab workspace for manipulation and analysis at a later time. As variables were needed for analysis and defining the figures depicted in this report, they were easily output to the Matlab workspace.

\section{SIMULATION CONDITIONS}

\section{APPROACH DEFINITIONS}

The desired approach paths are defined in the simulation using the runway axes. The $\mathrm{x}$-axis of the runway axes runs longitudinally down the length of the runway, the y-axis left and right of centerline, and the z-axis defines altitude. The curvature of the earth is not taken into account, meaning this is a local coordinate system; however, for analysis of approaches, use of a local coordinate system is acceptable. Approaches were simulated to runway 7 at the Santa Barbara Municipal Airport (KSBA).

The ILS path is defined as depicted in Figure 14. The localizer transmitter is positioned at the departure end of runway 7 , and is given a $3^{\circ}$ width either side of center. The glidepath transmitter is positioned at $1,000 \mathrm{ft}$ down the runway (the aiming point), with a glidepath of $3^{\circ}$ ( $6^{\circ}$ for STOL approaches), and a glidepath signal width of $0.7^{\circ}$ above and below center.

The SNI path for this test is defined as a decreasing $360^{\circ}$ spiral, as depicted in Figure 36. A straight segment of 0.3 miles before the runway threshold is included at the end of the spiral to give a bit of time for the aircraft to stabilize on final. The initial approach is offset from the runway by 0.75 miles. The SNI turn is commenced at the point abeam the start of the 0.3 mile straight segment. The radius of the turn is initially 1.5 miles, and is reduced smoothly to 0.75 miles by the end of the $360^{\circ}$ turn, approximating the lateral portion of an Ouija approach. To 
allow the use of the same glidepath coupler, the SNI approach is defined with a $3^{\circ}$ glidepath (or $6^{\circ}$ for the STOL approaches) based on the distance from the touchdown point around the curved path. Glidepath width is held at $0.7^{\circ}$ above and below center.

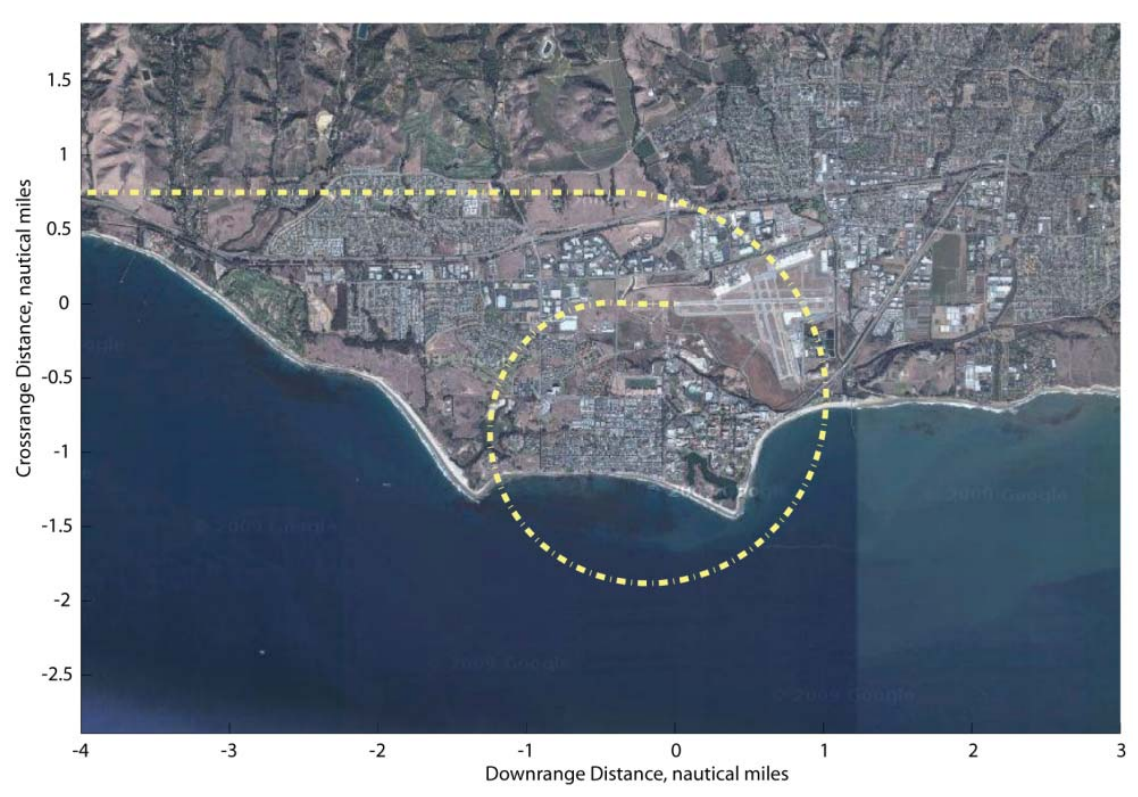

Figure 36 - SNI Testing Path

\section{INTERCEPT CONDITIONS AND AIRCRAFT CONFIGURATION}

All approaches were flown by intercepting the lateral navigation path while in level flight before intercepting the glidepath. This is a real world operational necessity; obstruction clearance is not ensured in the descent until the aircraft is positioned on the approach path. For the ILS approaches, the aircraft is flown to an intercept at an altitude of $1800 \mathrm{ft}$ (2500 ft for the steep ILS) at a distance of six nautical miles from the threshold, and an intercept angle of $15^{\circ}$. This is typical for an ATC vectored ILS intercept. Similarly, the initial segment of the SNI approach is intercepted at an altitude of $2500 \mathrm{ft}$, a distance of one to two nautical miles from the threshold, and an angle of $30^{\circ}$ (GPS steering computes its own intercept angle).

Two approach configurations are flown: conventional and STOL. For the conventional approaches, the aircraft is configured with outboard flaps set to $30^{\circ}$, USB flaps set to $0^{\circ}$, and 
autothrottles commanded at 120 knots. For the STOL approaches, outboard flaps are set to $59^{\circ}$, USB flaps are set to $30^{\circ}$, and autothrottles are commanded at 85 knots. Boundary layer control and spoiler DLC are utilized for both configurations. Heading and altitude hold autopilot modes are utilized until localizer and glidepath intercept (respectively), at which points the couplers take over.

\section{TEST CONDITIONS}

Four test conditions were run for each type of approach. The first tests were run in calm or "perfect” conditions, with no turbulence, wind, or windshear. This gives a baseline performance evaluation. The second set of approaches was run with moderate simulated turbulence to determine the effects of noise on the autopilot performance.

Next the approaches were run with a 30 knot direct crosswind. This evaluates the autopilots ability to establish a proper "crab" angle on the straight in approach and its ability to adjust for a changing groundspeed on the SNI approaches. The last set of approaches was run with a quartering headwind of 30 knots (at a $45^{\circ}$ angle to the runway) at 3,000 feet, reducing linearly to a wind speed of zero at ground level. This is designed to evaluate how both the lateral and glidepath navigation respond to changing wind conditions. The test conditions are summarized in Table 1.

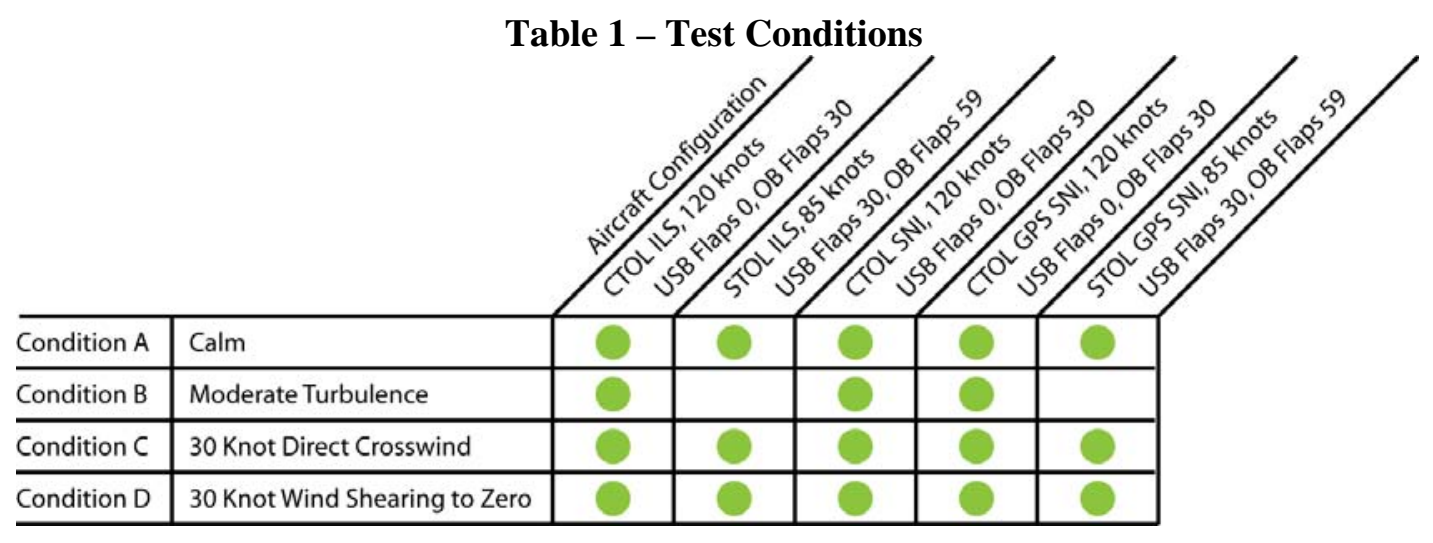




\section{RESULTS}

A total of 18 test cases were run, 7 of which were ILS approach cases. The remaining 11 cases were SNI approach cases. The ILS approach cases help provide a baseline for the approach coupler performance when compared with the SNI approaches.

For each of the five aircraft configurations, the results are shown in strip chart format, with range from the runway threshold plotted on the x-axis (for example, see Figure 37). The first plot depicts glidepath tracking. The standard angle between full range extents on an ILS glidepath is $1.4^{\circ}$; the angle on this plot has been significantly graphically expanded to provide a better depiction of glidepath performance. The limit lines shown on this plot are $0.07^{\circ}$ from center, corresponding to $10 \%$ either side of full deflection on glidepath. The Y-axis on this plot is analogous to altitude, but there is no direct correlation because the glidepath angle has been expanded.

The next plot shows the lateral tracking on the localizer. The localizer width is $3^{\circ}$ either side of center; the reference lines at $\pm 0.3^{\circ}$ correspond to $10 \%$ localizer deflection. A similar plot is shown for the SNI approaches, except rather than an X/Y plot, the crosstrack error is plotted against the range from the runway around the curved path: the SNI curve is effectively stretched out to a linear depiction. CDI sensitivity remains constant for the SNI approaches at RNP 0.3 nautical miles, so a localizer depiction is not included on the SNI lateral plots.

The remaining two plots depict variations in pitch attitude, airspeed, bank angle, and normal acceleration.

\section{CONVENTIONAL ILS APPROACH}

The performance on the CTOL ILS in calm conditions is shown in Figure 37. 

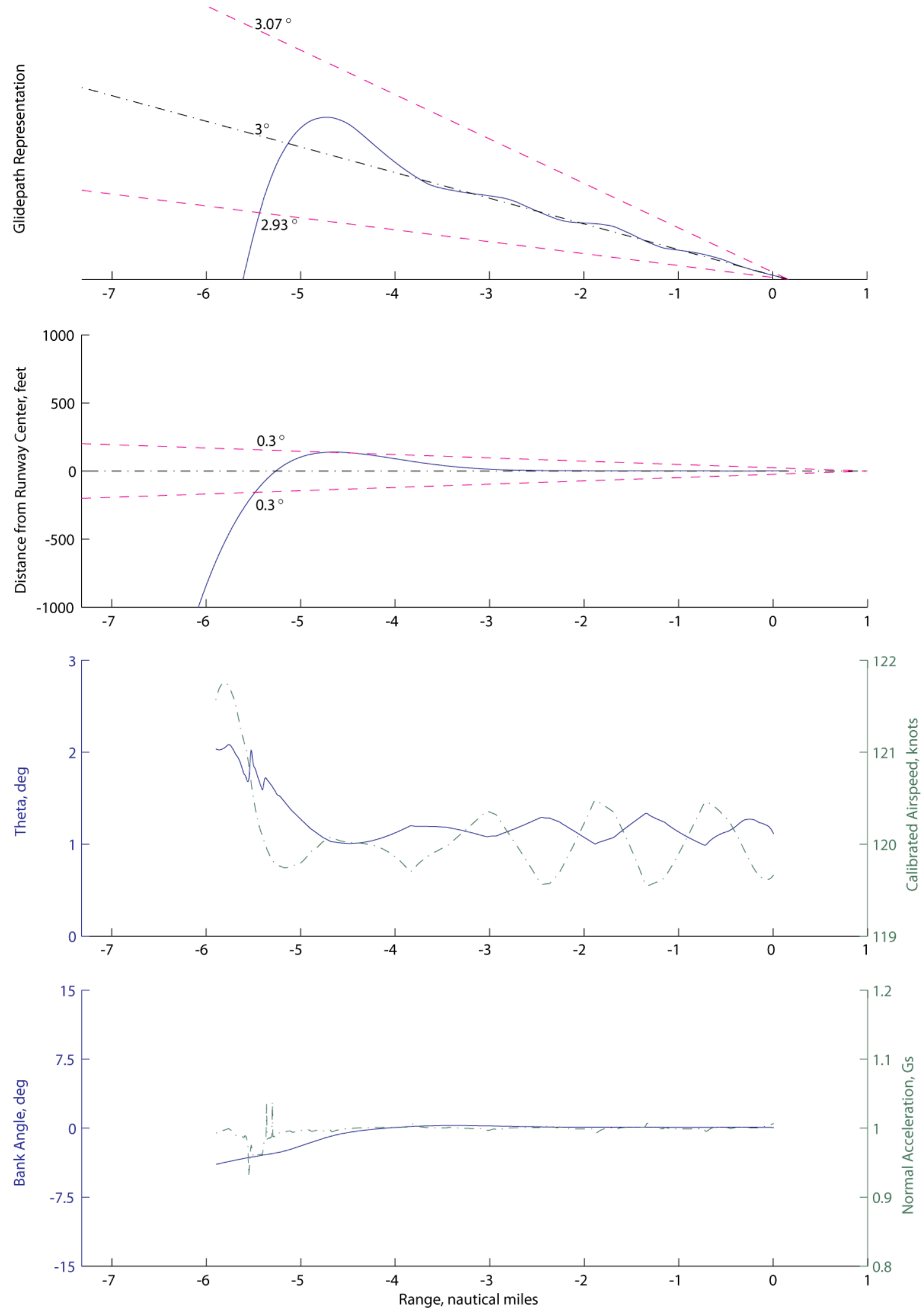

Figure 37 - CTOL ILS in Calm Conditions 
The controllers are quite acceptable for the conventional approach. Both glidepath and localizer intercepts occur with one small overshoot. A bit of oscillation is apparent on the glidepath, but the oscillation corresponds to about $1 \%$ of glidepath total deflection. Oscillation is apparent in pitch attitude and airspeed, but the magnitudes of these oscillations are small (on the order of one knot and $0.25^{\circ}$. A $180^{\circ}$ out of phase coupling is displayed between pitch and airspeed. G-force is $1 \mathrm{G}$ for the entire approach, excluding the small intercept transient.

Figure 38 depicts the glidepath performance for all four atmospheric conditions on the standard ILS approach.

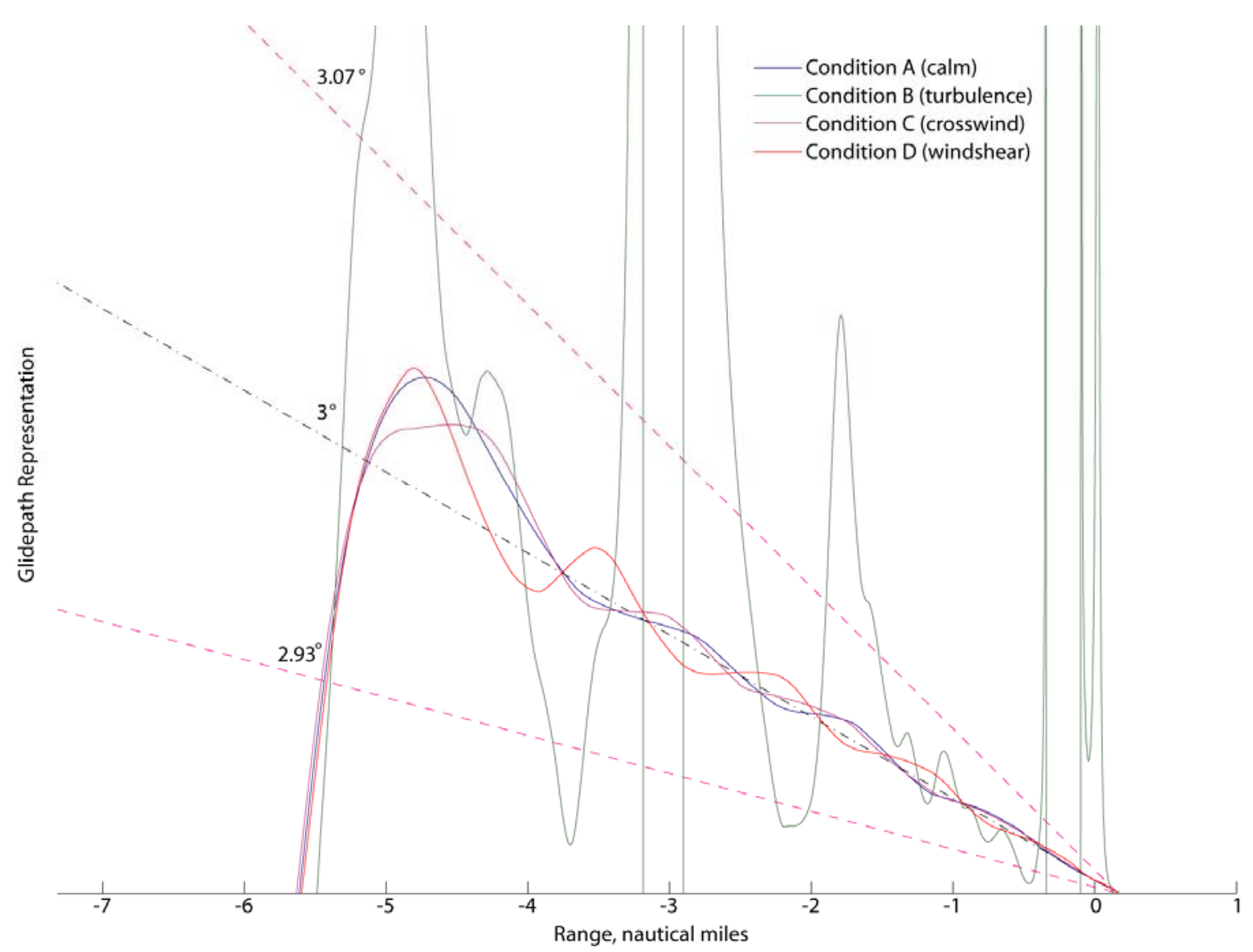

Figure 38 - CTOL ILS Glidepath Performance

The glidepath performance for Conditions A, C, and D is nominal, however significant glidepath excursions are obviously present for the Condition B, which corresponds to simulated "moderate" 
turbulence. Condition B does not plot well on the expanded angle plot, and is therefore shown in Figure 39, with glidepath error plotted as a percent of total deflection on the glidepath signal.

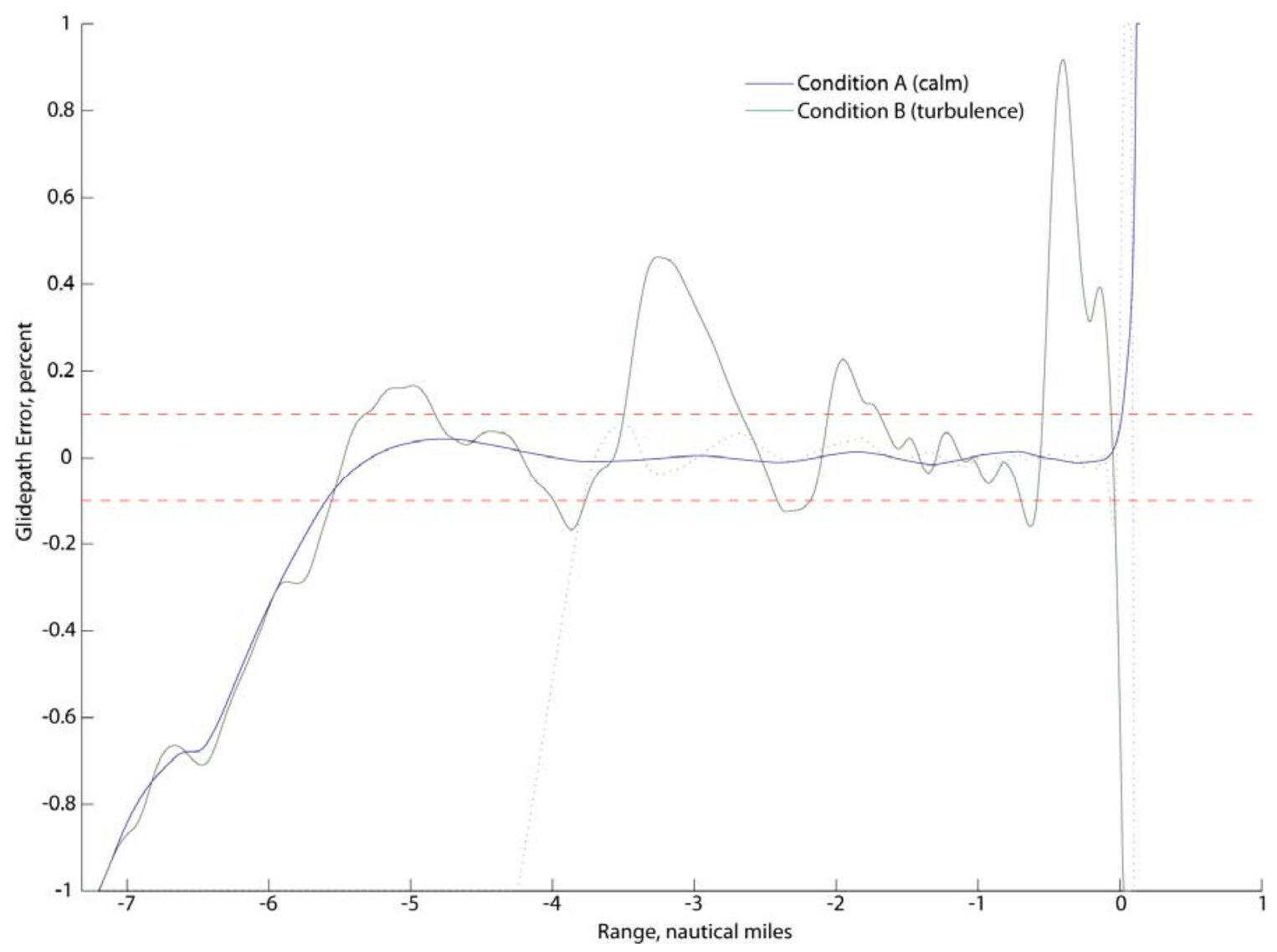

Figure 39 - CTOL ILS Turbulence Glidepath Performance

It can be seen that in moderate turbulence, the controller tracks the glidepath signal within onehalf deflection until about 0.5 miles from the landing threshold. While this would obviously not result in a successful autoland approach, it is probably typical of the type of glidepath tracking that could be expected from a "standard" autopilot in moderate to severe turbulence. Glidepath tracking for the turbulence case on the other approaches is quite similar to this case, and is therefore not included on the remaining glidepath tracking plots.

Figure 40 gives a comparison of lateral tracking in the different conditions on the standard ILS. 


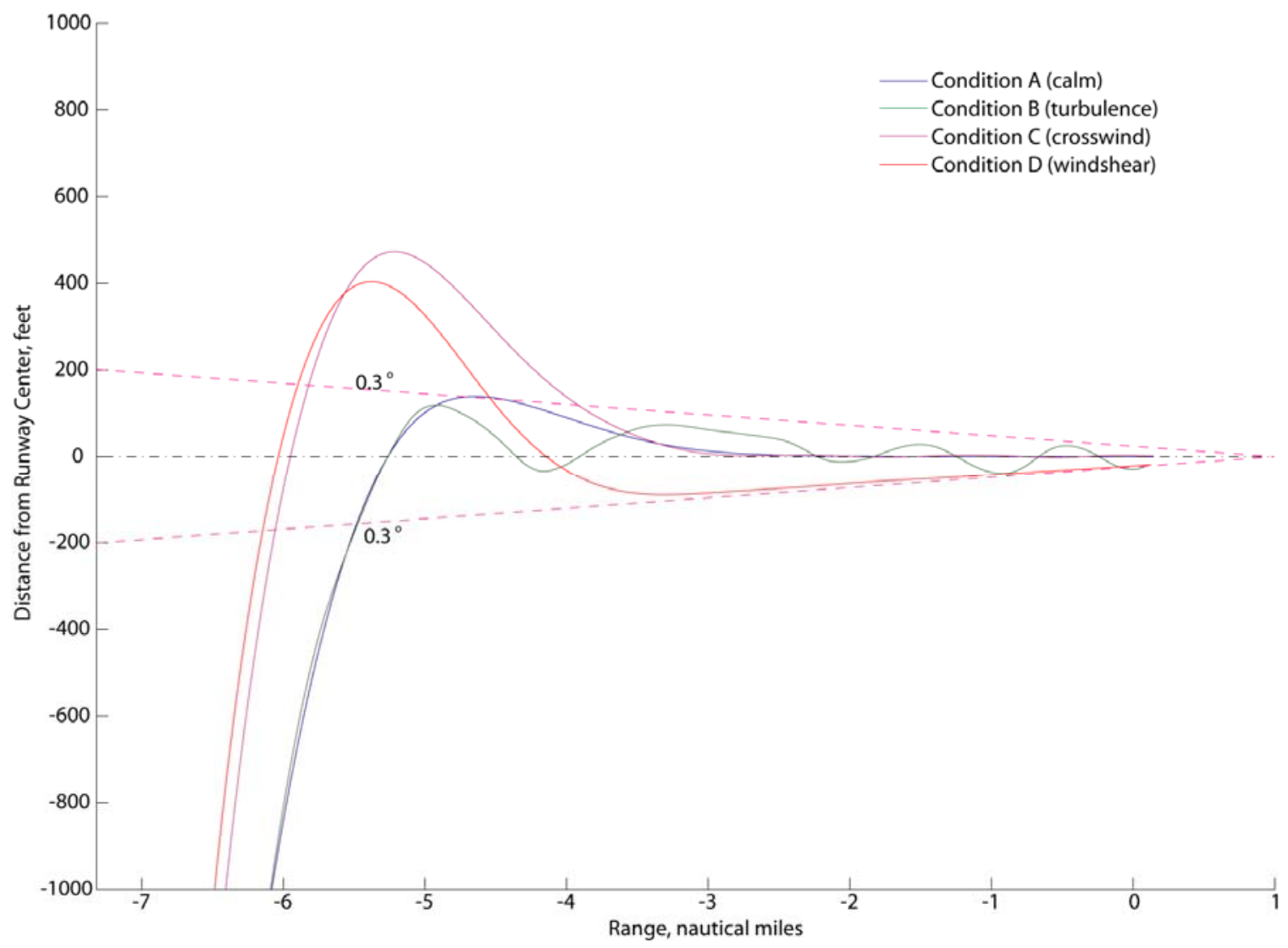

Figure 40 - Standard ILS Lateral Tracking

The lateral tracking cases all result in acceptable performance, with some minor differences. The turbulence case, as would be expected, results in somewhat unpredictable deviations from center. The crosswind case result in a larger overshoot (the crosswind is from the same side of the localizer as the initial intercept heading) than the calm case and a slightly longer settling time, but as the integral compensation kicks in to provide an appropriate crab angle, tracking settles in on center. The windshear case results in lateral tracking slightly off center: as the crosswind component velocity decreases, there is a slight time delay before the integral compensation can adjust the crab angle, resulting in tracking that is consistently slightly off center. The windshear tracking can still be considered adequate, as it tracks within 10\% localizer deflection.

Figure 41 depicts the pilot's view from the last $200 \mathrm{ft}$ on the ILS approach. These views are useful in determining how stabilized the aircraft is on each approach. 


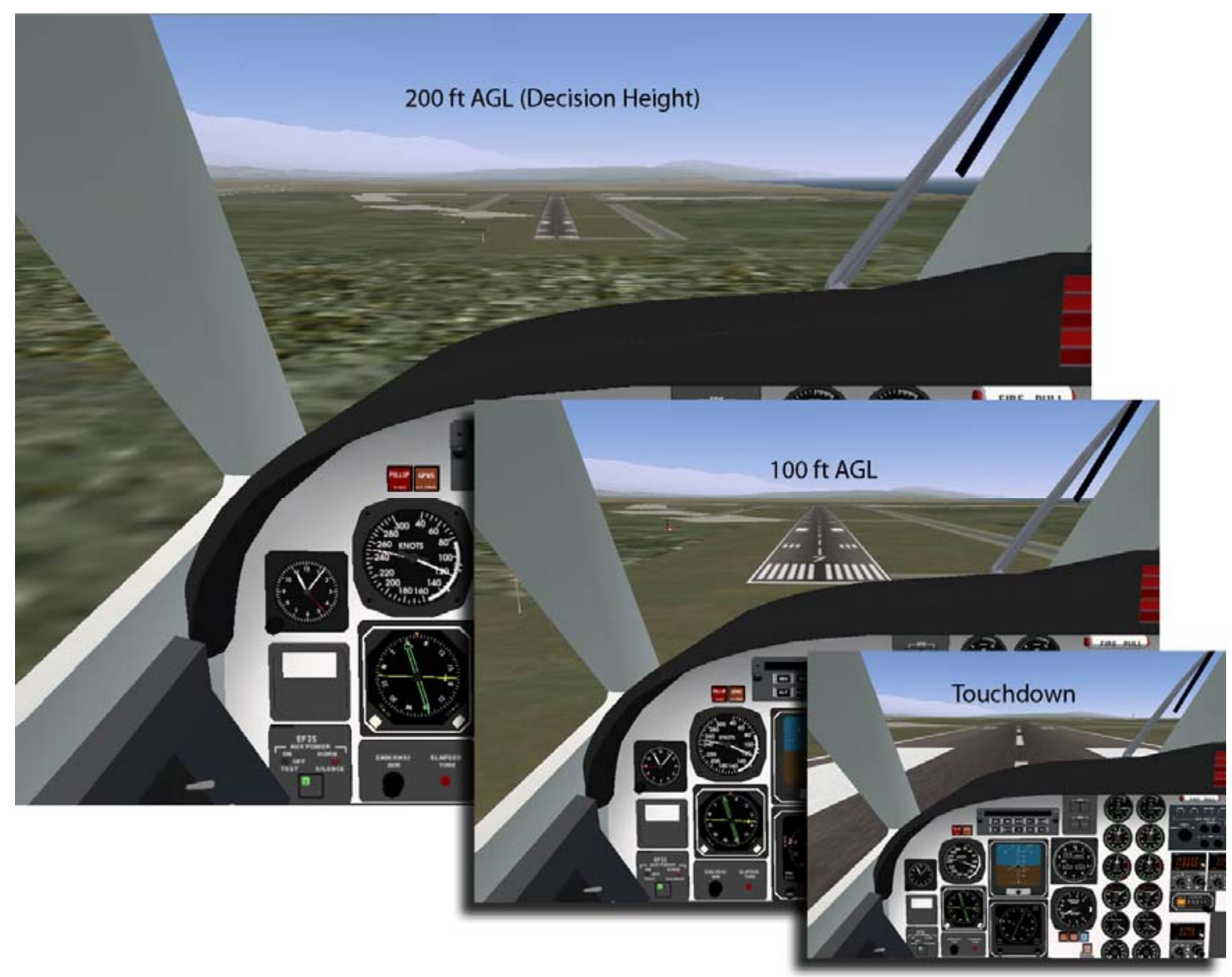

Figure 41 - Standard ILS View at Decision Height

The view at $200 \mathrm{ft}$ is typical of what a pilot would expect to see at the decision height on an instrument approach after just descending out of the cloud layer. At this point the pilot makes the “decision” as to whether to land the aircraft or conduct a missed approach. The criteria for continuing the landing is having the runway in sight and the aircraft in a stabilized configuration and capable of making a normal landing on the runway without excessive maneuvering. As can be seen by the sequence of images, the ILS approach results in a stabilized final approach segment, and even the rudimentary autoland system implemented in this simulator has no issue landing on target.

\section{STOL ILS APPROACH}

The tracking on the $6^{\circ}$ glidepath ILS at 85 knots in calm conditions is shown in Figure 42 . 

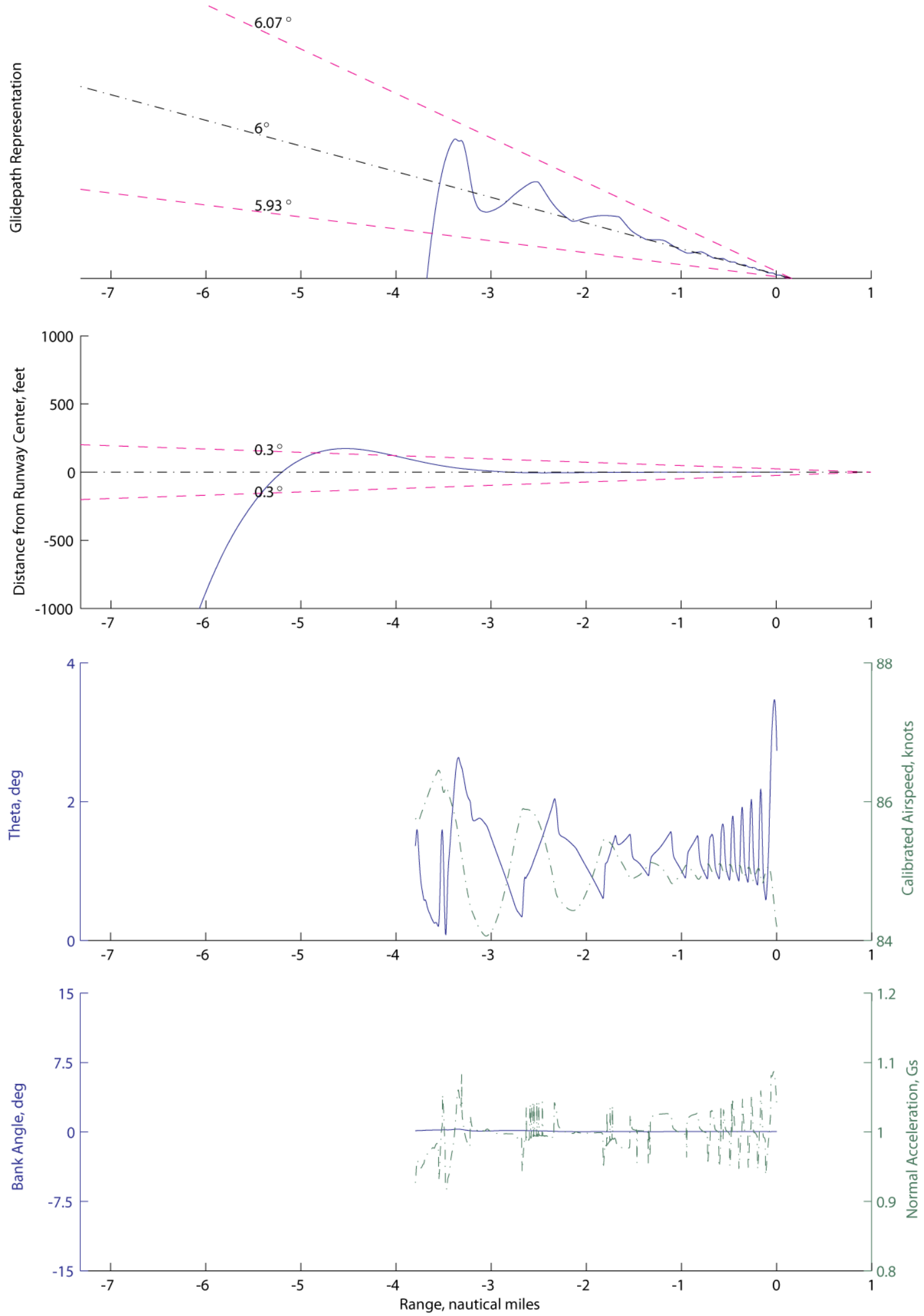

Figure 42 - STOL ILS in Calm Conditions 
The glidepath tracking in the STOL configuration on the $6^{\circ}$ glidepath is quite similar to the glidepath tracking on the $3^{\circ}$ glidepath at 120 knots. Lateral track intercept and tracking is similar as well. The main difference is understood when looking at the last two plots. Pitch angle oscillates between 0 and $2.5^{\circ}$ fairly consistently, and airspeed oscillates plus or minus one knot. These oscillations result in between 0.9 and 1.1 G-loading. Oscillation of this nature is undesired, and probably borderline acceptable.

Notice that airspeed and pitch angle are no longer $180^{\circ}$ out of phase. The reason for this oscillation is the increased interaction in the STOL configuration between pitch and power. With the USB flaps extended, thrust vectoring is occurring, and any change in power will result in an immediate flight path change. Thus, an ideal controller would consider pitch and power as a coupled system to control flight path and airspeed. This test, however, uses conventional, uncoupled controllers. This dataset displays a deficiency of conventional control systems to control STOL aircraft on a glidepath, regardless of making a circling or straight in approach.

As seen in Figure 43, STOL glidepath performance is virtually unaffected by wind conditions.

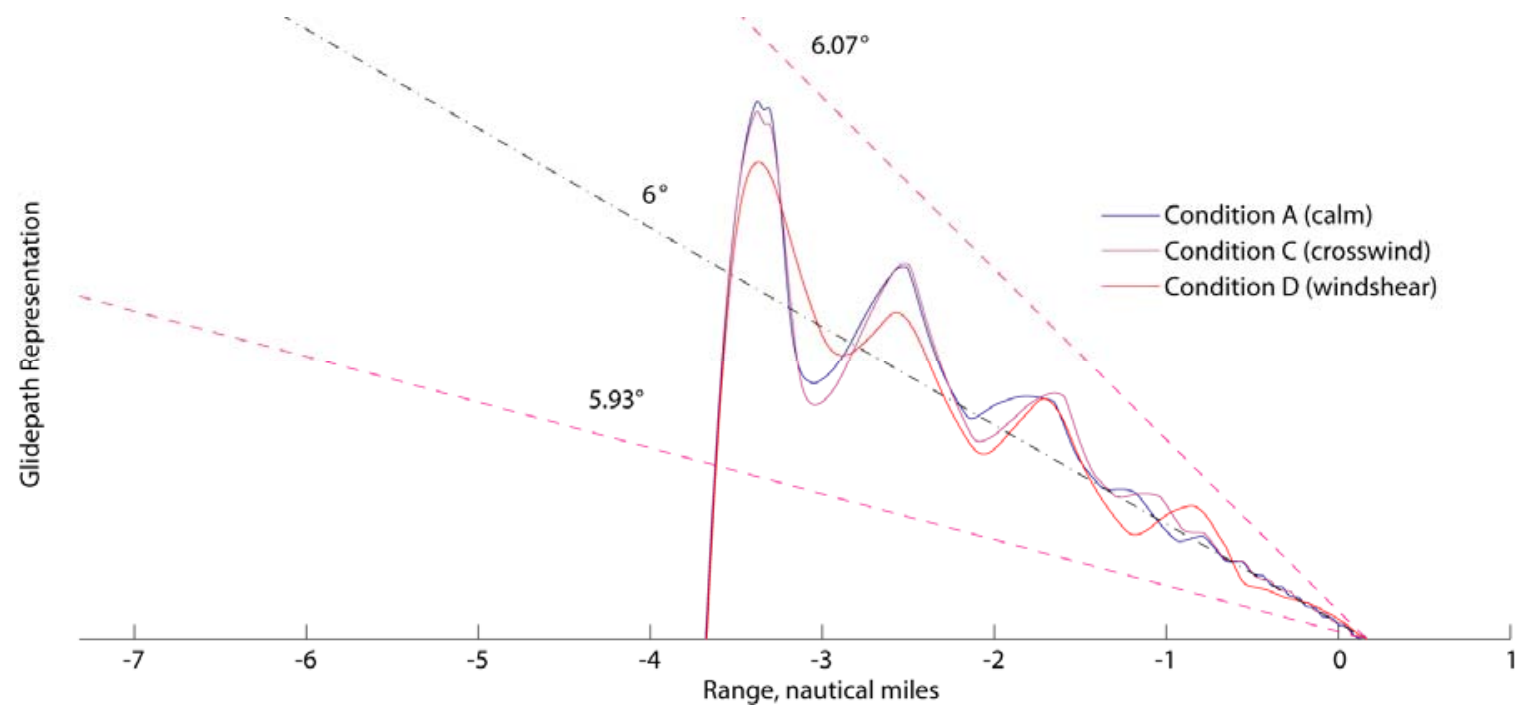

Figure 43 - STOL ILS Glidepath Performance 
The turbulence condition was not completed for the approaches at 85 knots because departures from controlled flight resulted. A coupled controller may be able to prevent this, but more than likely the approaches would need to be flown at a higher airspeed in turbulence in order to increase gust tolerance (as is common practice).

Localizer tracking in different conditions in the STOL configuration is nominally the same as the CTOL approach (Figure 44). The windshear case results in tracking slightly outside the arbitrary $0.3^{\circ}$ limit; this is due to slower aircraft speed with the same windshear gradient. Similar to the CTOL ILS, the STOL ILS results in a stabilized approach that can be landed from the approach minimums.

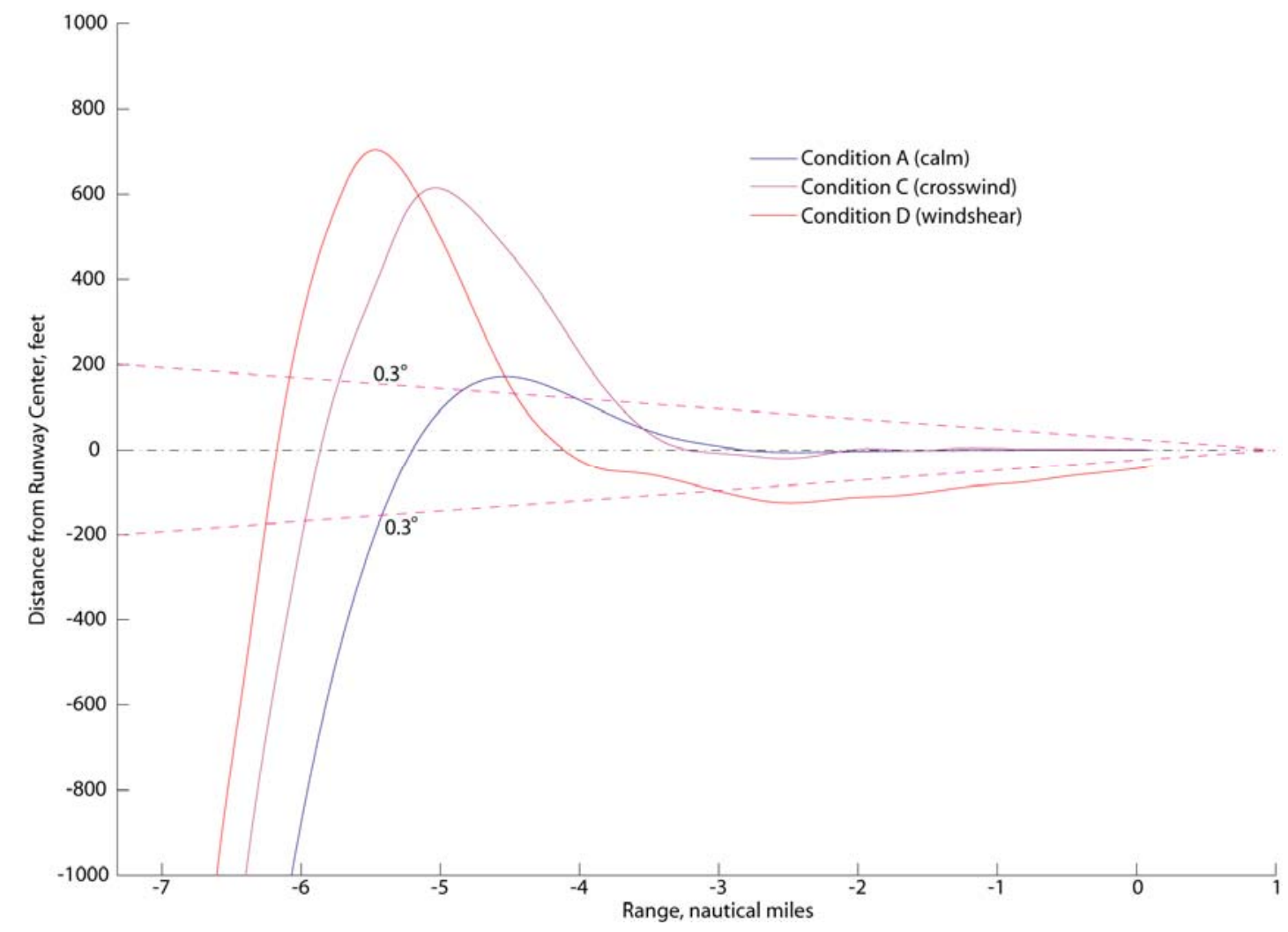

Figure 44 - STOL ILS Lateral Performance

\section{SNI APPROACH}

A $3^{\circ}$ SNI approach using the localizer lateral controller is shown in Figure 45. 

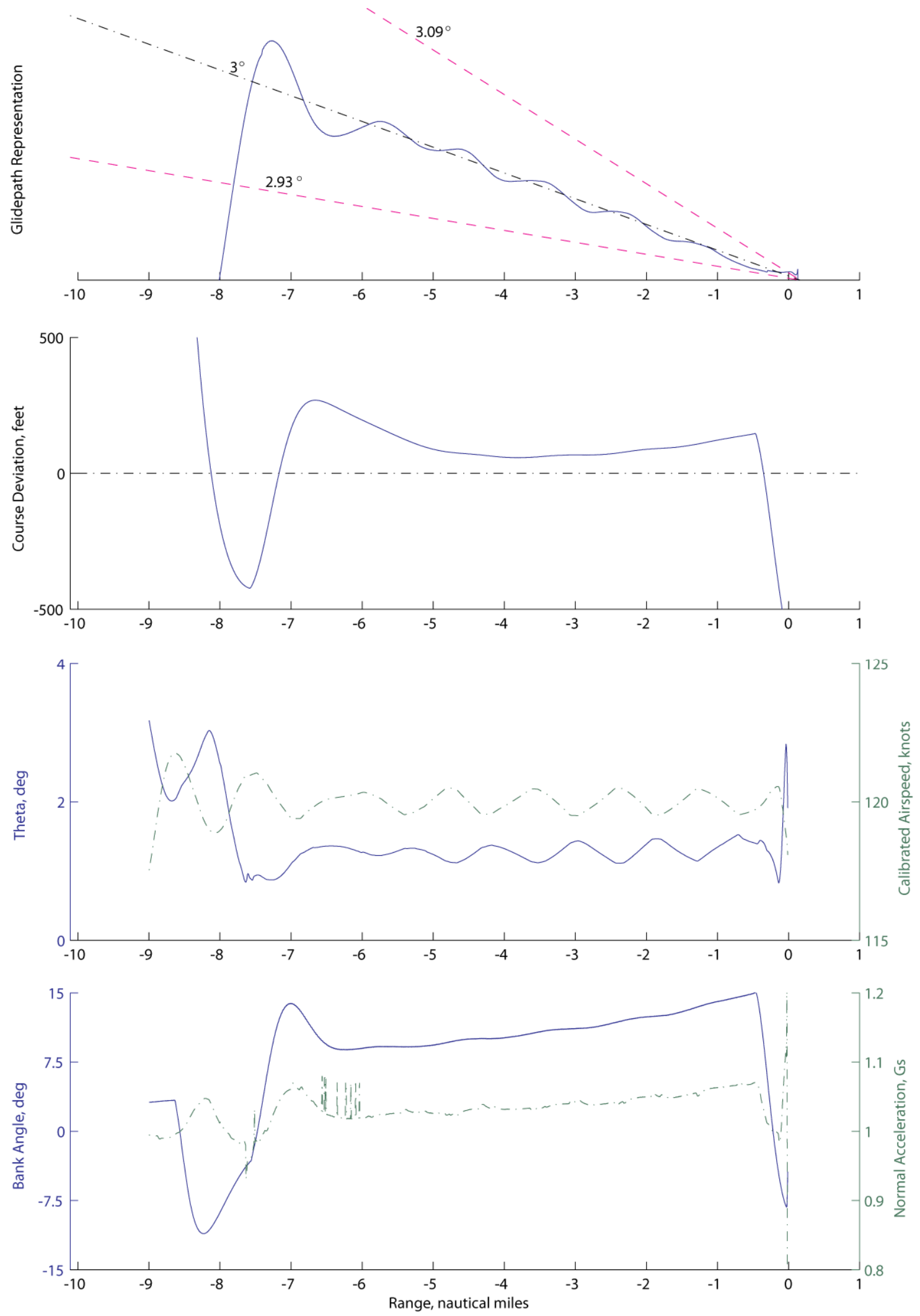

Figure 45 - CTOL SNI Approach with Localizer Coupler 
Interestingly, glidepath performance for the calm condition SNI approach is almost indistinguishable from the straight in approach. Lateral course tracking is much less precise on the SNI, however, than the straight in approach. After the initial lateral transient corresponding to intercept and then the beginning of the spiraling turn, the coupler does begin to settle on a deviation of less than 100 feet, but this deviation then begins to increase towards the end of the approach where the radius of the turn tightens. Most notable, however, is the sharp increase in crosstrack error that occurs at 0.3 miles from the runway threshold. This corresponds to the end of spiral; the integrator is "loaded" at this point and thus continues the turn even though the course ahead is straight. Airspeed and pitch oscillations are minimal, and a maximum bank of $15^{\circ}$ results in a load of nearly $1.1 \mathrm{Gs}$.

While glidepath performance on the SNI approach in calm conditions appears to be identical to the straight in approach, a disparity is noted when comparing the glidepath performance in the crosswind and windshear conditions (Figure 46). While probably still acceptable, these approaches show significantly more oscillation on glidepath and reduced tracking precision. This is because the glidepath coupler must compensate for changing groundspeeds as well as changing bank angles as the localizer coupler struggles to keep up with the changing conditions.

Actual ground track for all cases is shown in Figure 47. Note that the tracking for conditions A \& B appears to be acceptable right up until the end of the spiral where the aircraft continues to turn instead of making a rollout on heading. Cases C \& D, however, result in crosstrack errors in excess of 1,000 feet, illustrating the inability of the localizer coupler to quickly adjust its wind correction around a curved path.

The view from decision height on the SNI approach with the localizer coupler is shown in Figure 48. 


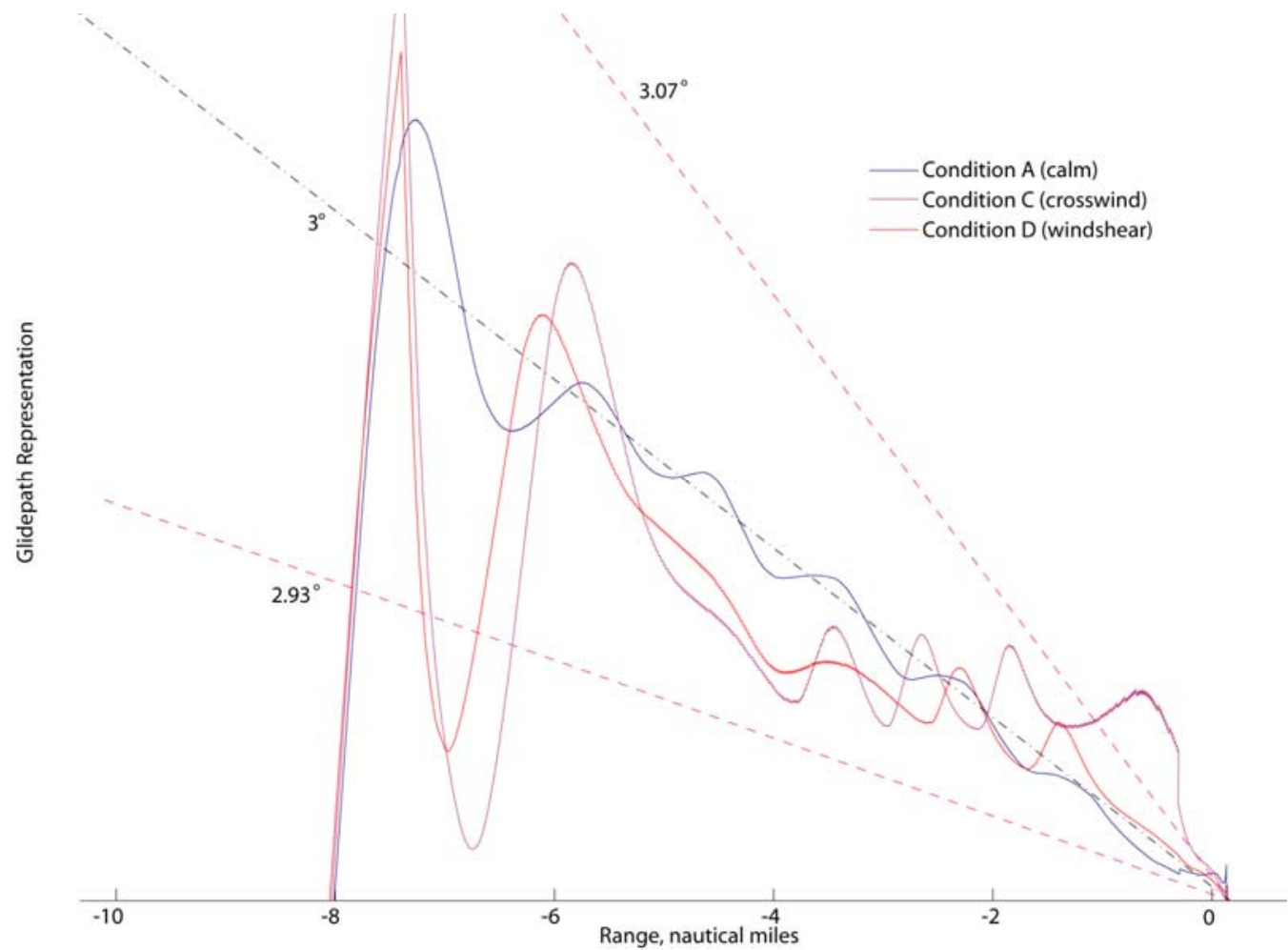

Figure 46 - SNI Glidepath Performance with Localizer Coupler

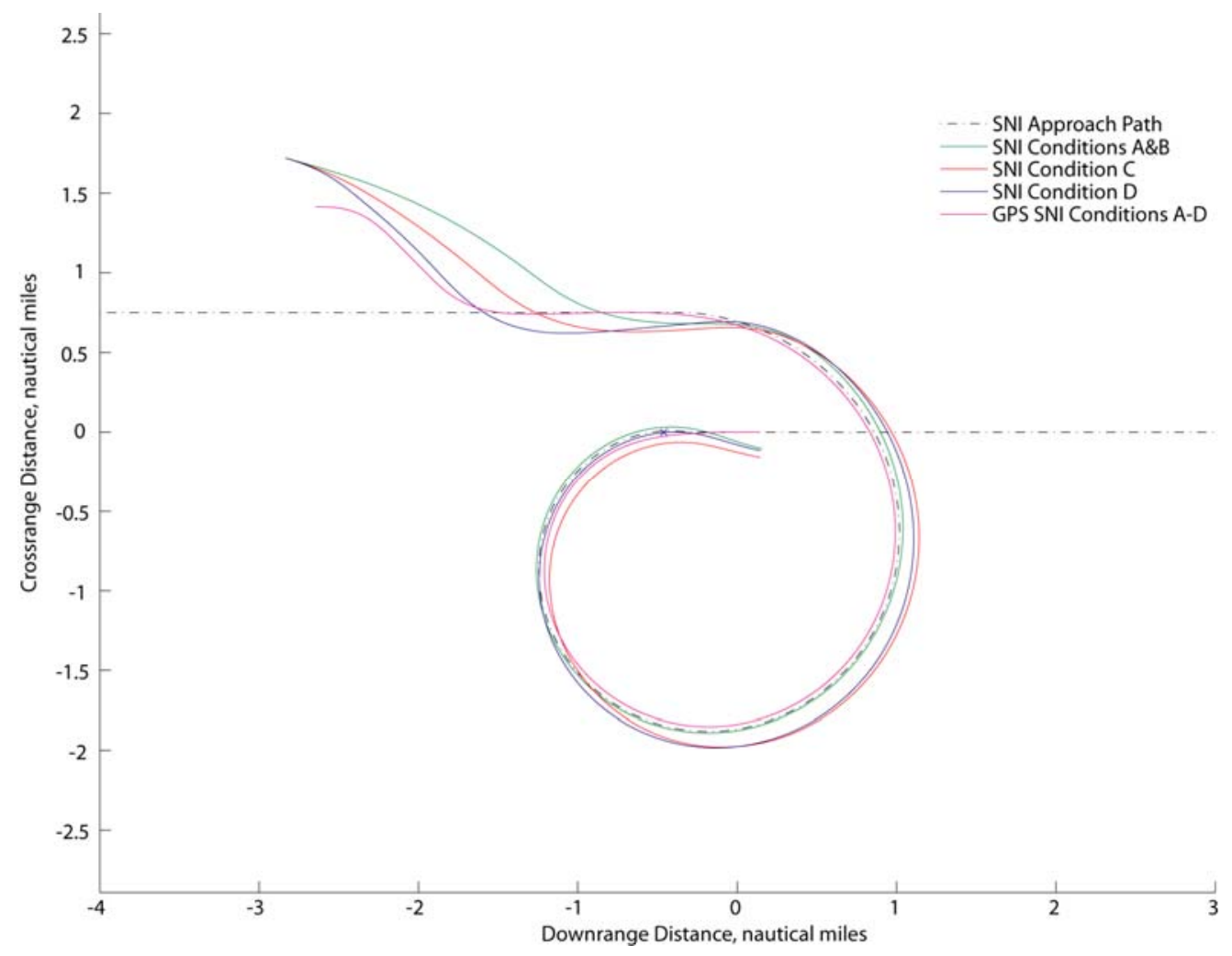

Figure 47 - SNI Lateral Performance 


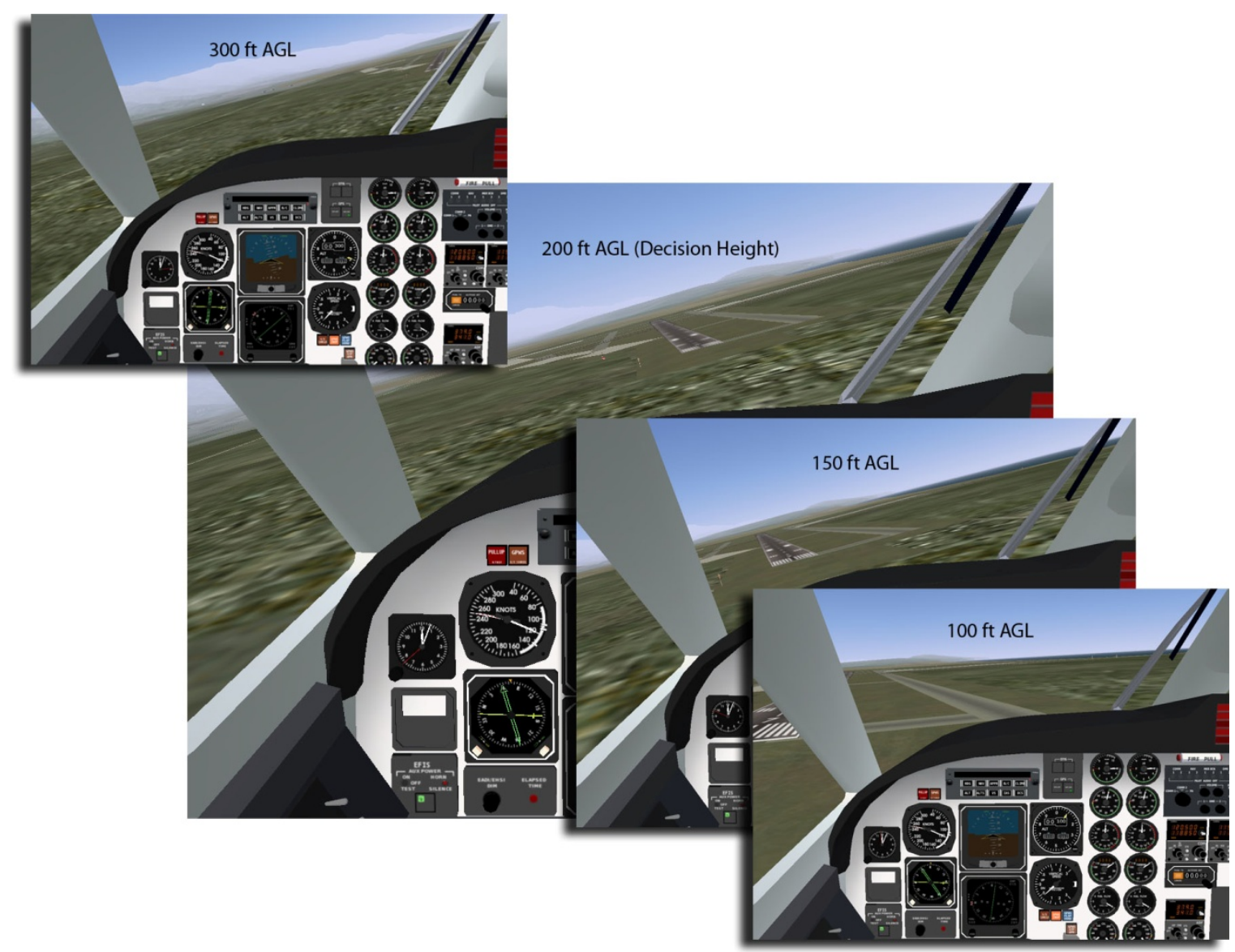

Figure 48 - SNI with Localizer Coupler View at Decision Height

While the cockpit view at $300 \mathrm{ft}$ appears to show the aircraft setting up for a successful rollout on final, the remainder of the images depict a situation to the contrary. At the $200 \mathrm{ft}$ decision height, the aircraft has crossed through the final approach, and it remains in the right banked turn as if to correct. At $150 \mathrm{ft}$, the aircraft is crossing through the final approach course a second time, still in a right bank. At $100 \mathrm{ft}$, the localizer controller has rolled the wings nearly level, but the aircraft is well to the right of the runway and on a divergent heading. Even considering an autopilot disconnect at decision height, this performance is unacceptable for an IFR approach, because the aircraft is not stabilized at $200 \mathrm{ft}$ and would require aggressive maneuvering in order to land on the runway. 


\section{GPS SNI APPROACHES}

Tracking on the CTOL SNI approach using GPS steering is depicted in Figure 49, and Figure 50 depicts tracking of the STOL SNI approach with GPS steering. Glidepath tracking for both cases is similar to the corresponding straight in ILS approach. Lateral course tracking results in a maximum track error of about 300 feet, but most importantly, the aircraft track rejoins the approach courses perfectly at the threshold rather than diverging from it. This is also illustrated in Figure 47, where all of the GPS SNI cases (CTOL \& STOL) can be represented by one path, because they are indistinguishable on this plot. This is because the GPS coupler automatically compensates for variations in wind speed and direction: all turn commands are driven by ground track. Thus, in terms of predictability, the GPS steering is far superior to the localizer coupler.

The CTOL GPS approach results in a maximum bank angle of about $15^{\circ}$, similar to the localizer coupled SNI approach. The STOL GPS approach, however, results in a maximum bank angle of about $7^{\circ}$, which corresponds to just more than $1 \mathrm{G}$ normal acceleration. With respect to bank angle, this approach would likely be more comfortable for passengers. The 85 knot approach on a $6^{\circ}$ glidepath, results in a stabilized descent rate of about $950 \mathrm{ft}$ per minute, whereas the $3^{\circ} 120$ knot approach results in a descent rate of about $600 \mathrm{ft}$ per minute. The increased descent rate and descent angle would probably, at least initially, be disconcerting to both passengers and flight crew. 

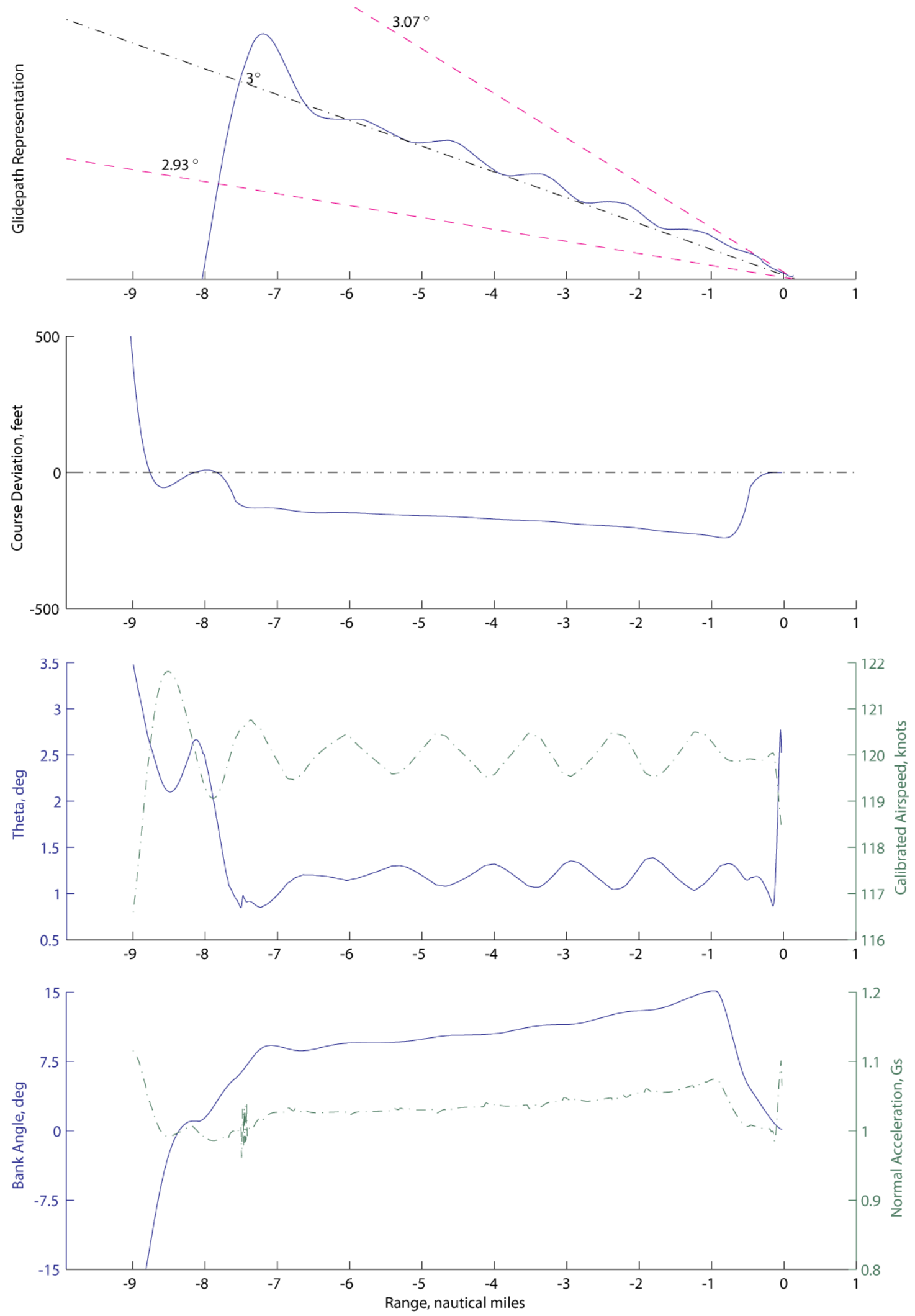

Figure 49 - CTOL GPS SNI Approach 

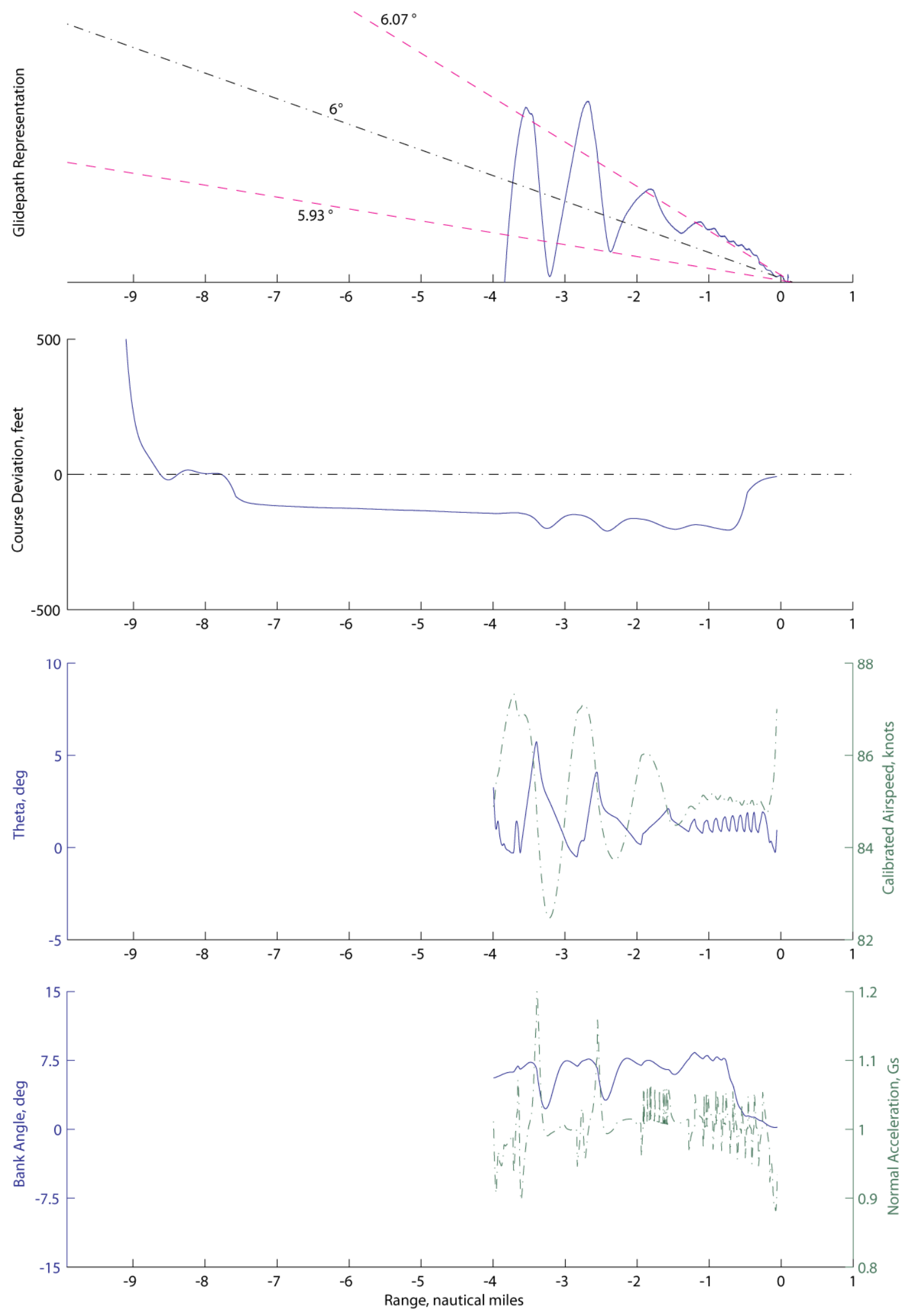

Figure 50 - STOL GPS SNI Approach 
Figure 51 depicts glidepath performance for the STOL GPS SNI approach. Glidepath performance is slightly improved over the case using the localizer coupler. It must be inferred that because the GPS coupler remains more stabilized on the lateral path (i.e., the bank angle curve is smooth), the glidepath coupler doesn’t have to work as hard to maintain adequate tracking.

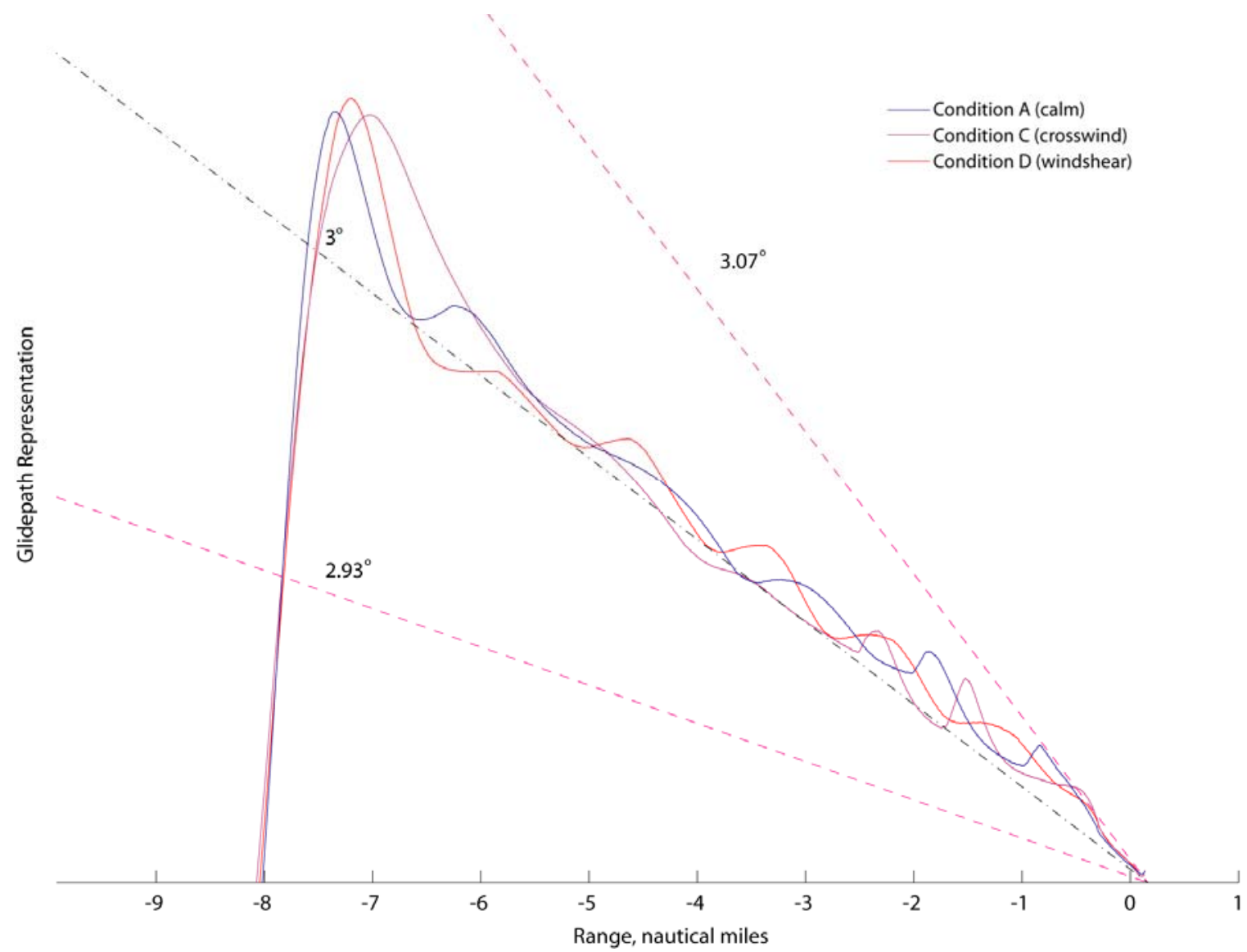

Figure 51 - CTOL GPS SNI Glidepath

Figure 52 depicts glidepath performance for all cases on the STOL SNI GPS approach. As compared to the STOL straight in approach, glidepath deviations are significantly greater in magnitude (though still acceptable). This is again an indication that adding the spiraling turn does have an effect on glidepath tracking, especially with STOL approach configurations and speeds. The effect, however, is not as great as one might have expected, probably due to the shallow bank angles involved. 


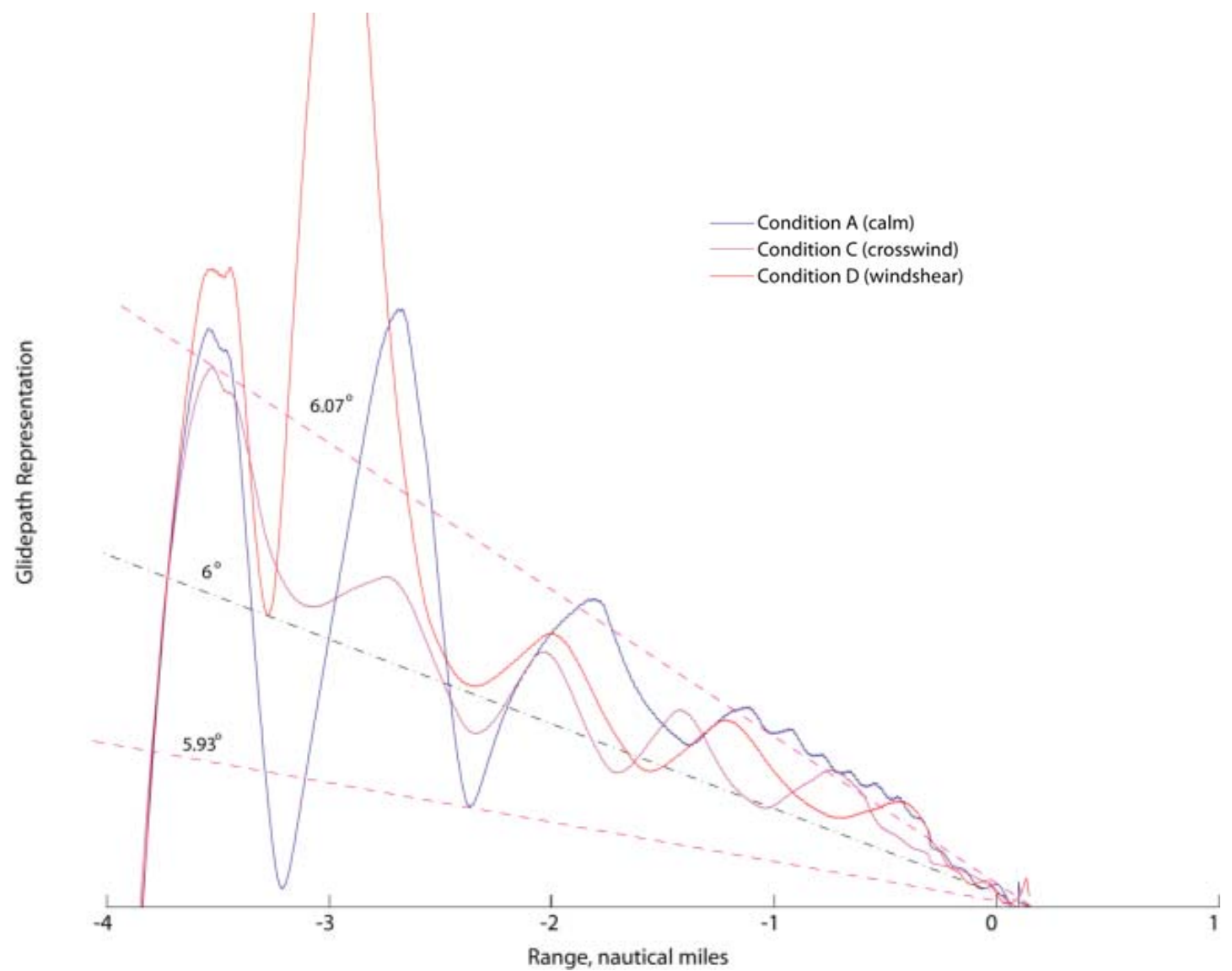

Figure 52 - STOL GPS SNI Glidepath

Figure 53 depicts the rollout performance of the GPS SNI coupler. At $300 \mathrm{ft}$, the view looks similar to the view when the localizer coupler was used. At decision height, however, it becomes aparent that the GPS coupler is commanding a rollout right on centerline. The $100 \mathrm{ft}$ and touchdown images show that from decision height, simply continuing the smooth rollout results in the aircraft tracking exactly along centerline. This is likely compliant with IFR guidelines for a stabilized final approach to landing, though pilots would need to become accustomed to "looking" for the runway slightly off center as well as timing the final portion of the rollout correctly. 


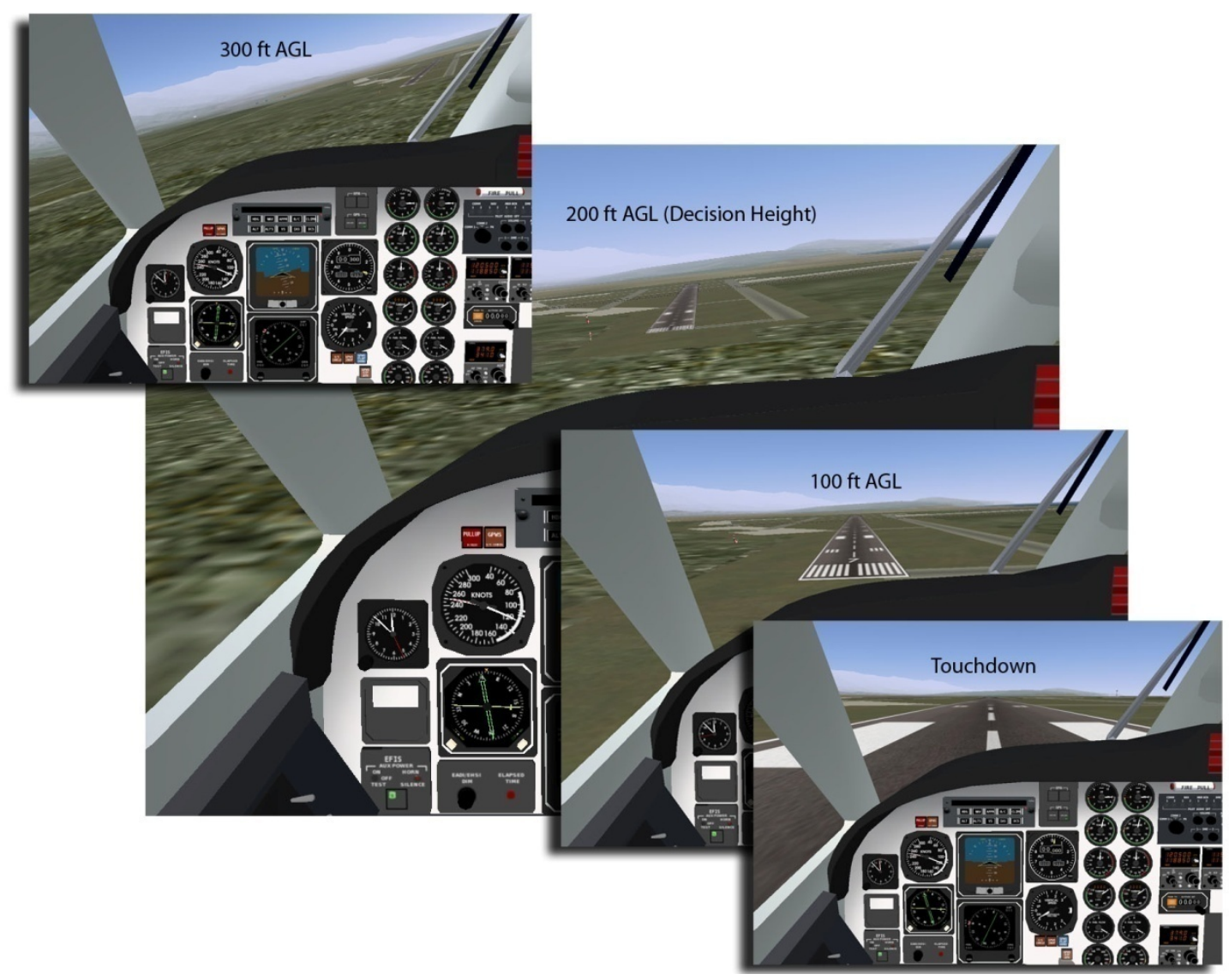

Figure 53 - CTOL GPS SNI View at Decision Height

Figure 54 depicts the STOL GPS SNI approach, which is quite similar to the CTOL GPS approach, with the exception of the steeper approach angle. Note that the deck angle between the two approaches is nearly identical. On the STOL approach, when the aircraft arrives at the $200 \mathrm{ft}$ $\mathrm{DH}$, the aircraft is nearly fully aligned on the runway; due to the steeper approach angle, the aircraft arrives at the decision height further along the approach path.

At decision height, the aircraft is nearly over the threshold of the runway, and the descent rate is stabilized at approximately $900 \mathrm{ft}$ per minute. This means that if the runway environment is sighted at exactly $200 \mathrm{ft}$ AGL, the flight crew has about 13 seconds to make any approach adjustments before landing. Comparatively, an approach at 135 knots on a $3^{\circ}$ glidepath results in 
a descent rate of $715 \mathrm{ft}$ per minute, and 17 seconds from arrival at minimums to touchdown (ignoring flare).

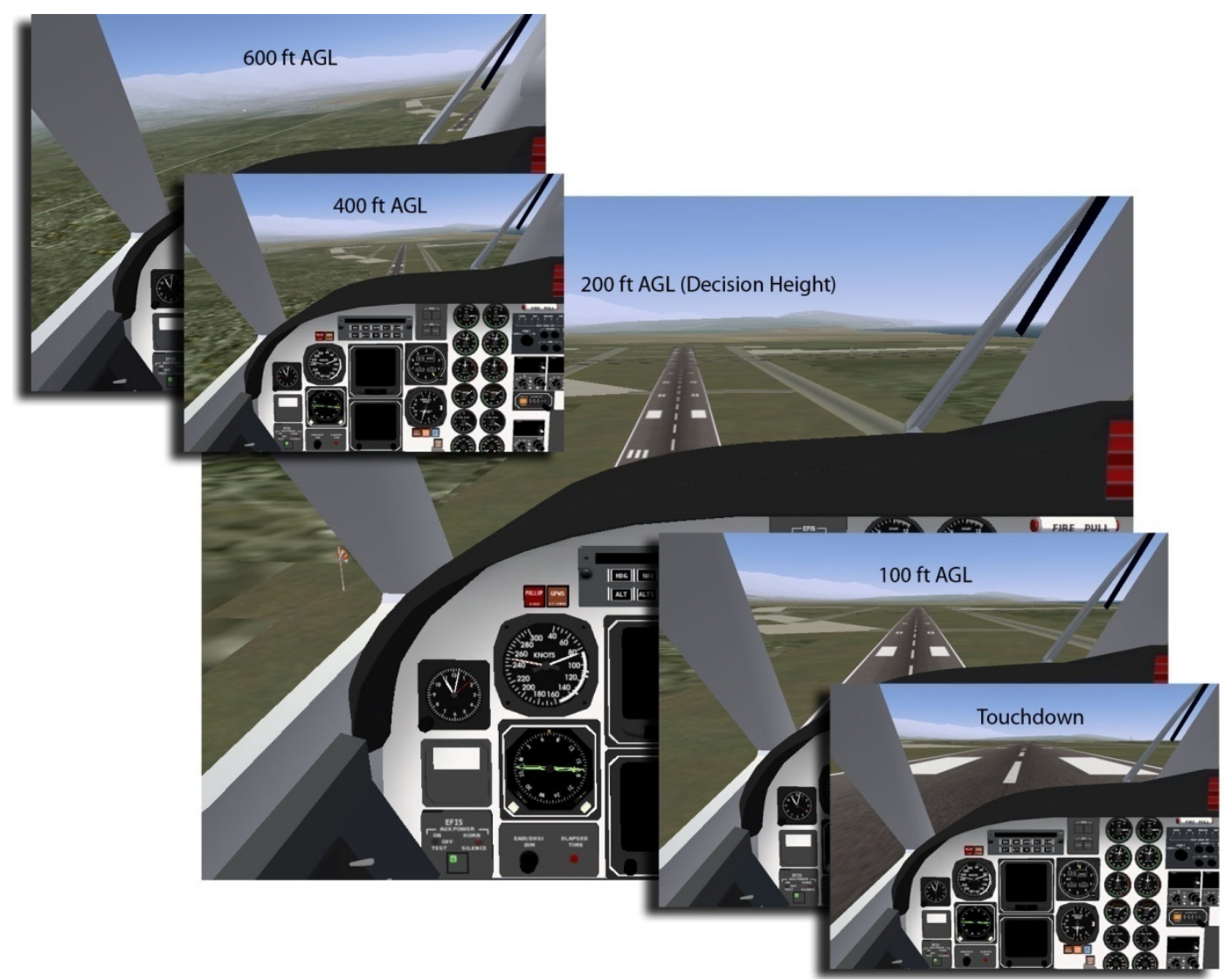

Figure 54 - STOL GPS SNI View at Decision Height

The decreased time available to the pilot to maneuver the aircraft from the decision height, coupled with the likely altitude loss from the initiation of a missed approach maneuver to achieving a positive rate of climb on a STOL approach may well require higher approach minimums. This may be the case for either a SNI or straight in STOL approach, though the straight in approach is less likely to require any maneuvering past the decision point. Nonetheless, the need for the approach coupler to guide the aircraft on target to minimums is underscored. 


\section{CONCLUSIONS}

Approach simulations were conducted with a replica of the Quiet Short Haul Research Aircraft Math Model, dubbed the PolyQSRA. CTOL and STOL configurations were evaluated for both straight in and circling (SNI) approaches. The goals of the simulations were as follows:

- Assess ability of current generation autopilots to track a SNI approach course in an IFR environment

- Assess the challenges of designing approach couplers for IFR use with STOL configured aircraft

- Assess the viability of the SNI approach as an IFR procedure

These objectives have been completed, and for the most part it appears that the SNI approach is viable given a new generation of technology. The following addresses the findings in each of the assessment areas.

\section{AUTOPILOT COUPLER ON THE SNI APPROACH}

The PID based glidepath and localizer couplers were capable of providing accurate and predictable approach path guidance on the ILS approaches. Glidepath performance was slightly oscillatory, especially for the steeper STOL approaches, but this would likely be the case for any conventional controller when paired with STOL aircraft dynamics.

When utilized on the SNI approach, the PID glidepath controller performance was nearly equivalent to the ILS baseline performance. This result is somewhat surprising, as it was expected that the addition of a turn during the approach would significantly increase the demands on the glidepath controller. 
The PID lateral coupler, however, proved to be inadequate for use with a SNI approach. In calm conditions, the performance of the coupler is marginal throughout the approach, and the aircraft misses its heading, track, and stabilized approach targets at the approach minimums. In the crosswind and windshear conditions, the lateral tracking of the PID coupler was even less acceptable: the coupler is not well suited to coping with rapidly changing conditions.

The GPS lateral coupler, however, shows significant promise to provide adequate performance on the SNI approach. The implementation of the GPS coupler utilized for these approaches will always result in a ground track slightly "inside” the desired track, but this could easily be fixed with a more advanced algorithm. More importantly for this test, predictability of ground track was demonstrated, as the ground tracks for all of the GPS coupled approaches are virtually indistinguishable. Likewise, the feed-forward system employed by the GPS coupler always results in a rollout onto a stabilized final approach course. GPS opens up the possibility of utilizing feedforward for glidepath tracking as well; much like the PolyQSRA implementation of GPS steering, flight path could be "managed” so that the aircraft was always traveling towards a future glidepath target. The sensitivity issues close to the threshold could in this way be avoided, and oscillations on glidepath would likely be reduced or eliminated.

The conclusion to be drawn, therefore, is that while current generation autopilots will not be capable of flying a SNI approach with adequate accuracy, it is possible to design next generation autopilots that will have no problem flying the SNI approach. Due to the complexities of flying the approach, the author maintains that autopilot use to the missed approach point would probably be a requirement in IMC. 


\section{APPROACH COUPLERS WITH STOL CONFIGURED AIRCRAFT}

In designing the approach couplers for PolyQSRA, it became apparent that designing an autopilot for use with a STOL aircraft presents significant challenges not present with CTOL aircraft. In the conventional configuration (USB flaps up), conventional controllers (autothrottle and glidepath coupler) are adequate for controlling the QSRA. However, with even small extensions of the USB flaps (and the slower resultant speeds), significant inadequacies become apparent.

Even in conventional aircraft, an obvious coupling exists between pitch and power required to maintain a specified airspeed. If a climb is commanded, the pitch controller raises the nose, and then the autothrottle (sensing a decrease in airspeed) increases throttle setting to maintain the preset airspeed. Pitch attitude and power setting for a given flightpath and airspeed combination are approximately $180^{\circ}$ out of phase. As such, when one controller initiates a change, the other controller is able to sense the change in initiate a corresponding change before a significant deviation occurs.

With the QSRA in the STOL configuration however, the USB thrust vectoring results in a nearly in-phase relationship between pitch and power setting. A climb commanded by raising the nose initially results in a significant increase in drag without an appreciable altitude gain. The increase in drag results in a command to the autothrottle to increase power, which has less than the desired effect on airspeed but results in a significant increase in climb rate. This in turn results in a reduction in pitch attitude to correct, and so on. This phenomenon is noticeable in the STOL configuration when simple climbs, descents, or level accelerations are commanded (Figure 34), but is more pronounced with the glidepath coupled, due to the additional loop closure and associated time delay. 
Thus, while the conventional glidepath coupler utilized in this simulator was adequate (marginally) for the STOL simulations conducted, a more advanced controller would be required for STOL aircraft configurations to be accepted by pilots, passengers, and the FAA. The controller would need to be capable of making a simultaneous adjustment of pitch and power setting to obtain a desired resultant flightpath and airspeed in all aircraft configurations (i.e. a multi-input, multi-output system). Design of this controller is beyond the scope of this thesis, but it could probably be accomplished either by the use of adaptive controllers or by performing system identification on the STOL configuration and developing a meta-model that would give control modulations necessary to achieve a desired result. This type of controller would be absolutely necessary in order to fly the "continuously decelerating” SNI approach, as is the ultimate vision of the approach.

\section{SNI APPROACH VIABILITY}

The SNI approach appears to be a viable IFR approach concept. Implementation will require new avionics equipment, but this should not be considered a significant issue, as the vision requires a new generation of STOL aircraft to utilize the approach. Based on the shallow bank angles of the approach and low G-forces observed, passenger acceptance of the approach is likely to be a nonissue (though the view out the side window of the aircraft in a bank at $300 \mathrm{ft}$ AGL might be somewhat disconcerting).

The greater roadblocks to acceptance of the approach are likely to be pilots and the FAA. Both have the concept ingrained (with good reason) that all approaches must be stabilized and on centerline by 1,000 ft AGL for IFR landings. Thus, an approach that results in a rollout on centerline at $200 \mathrm{ft}$ AGL, requiring a simultaneous land or missed approach decision may not sit well. This is why approach path predictability on autopilot is so crucial to the ultimate success of 
the SNI approach. Nonetheless, this ability to achieve a predictable approach path has been demonstrated.

Questions remain as well as to whether the SNI approach would actually result in increased airport capacity. This would be dependent on the acceptance by the FAA and also pilots (and the general public) of operating aircraft with decreased separation in the IFR environment while utilizing a new procedure. Once again, integral in the FAA's decision would be the demonstration of extremely precise flight paths on the SNI approach, which have been demonstrated by this thesis. 


\section{WORKS CITED}

1. Boeing Commercial Airplane Company. Quiet Short-Haul Research Aircraft Phase II Flight Simulation Mathematical Model - Final Report. Moffett Field : NASA Ames Research Center, 1979.

2. McFarland, Richard E. A Standard Kinematic Model for Flight Simulation at NASA-AMES. Washington D.C. : National Aeronautics and Space Administration, 1975. NASA CR-2497.

3. Norton, Bill. STOL Progenitors: The Technology Path to a Large STOL Aircraft and the C17A. Reston : American Institute of Aeronautics and Astronautics, 2002.

4. The Boeing Company, Commercial Airplane Division. QSRA Operations Manual. Renton : Boeing, 1978. Manual.

5. Hange, Craig and Eckenrod, Dennis. Assessment of a C-17 Flight Test of an ESTOL Transport Landing Approach for Operational Viability, Pilot Perceptions and Workload, and Passenger Ride Acceptance. Reno : 45th AIAA Aerospace Sciences Meeting and Exhibit, 2007. AIAA 2007-1398.

6. Fong, Lauren Elizabeth. Simultaneous Non-Interfering Approaches Using Integrated Noise Modeling for the C-17 Noise Mitigating - Smart Terminal Area Trajectory Flight Test. San Luis Obispo : California Polytechnic University, 2005. Senior Project.

7. Hange, Craig. C-17 Noise Mitigating Smart Terminal Area Trajectory. Grapevine, Texas : International Powered Lift Conference, 2005. Powerpoint. 
8. U.S. Department of Transportation, Federal Aviation Administration. Aeronautical Information Manual. Pittsburgh : U.S. Government Printing Office, 2008.

9. Federal Aviation Administration. The Federal Aviation Regulations. 2008.

10. Flight Gear. [Computer Program www.flightgear.org] 2009. 


\section{Appendix I - 6DOF Point Mass Validation}

Linear validation for a unit mass, typical for all directions.
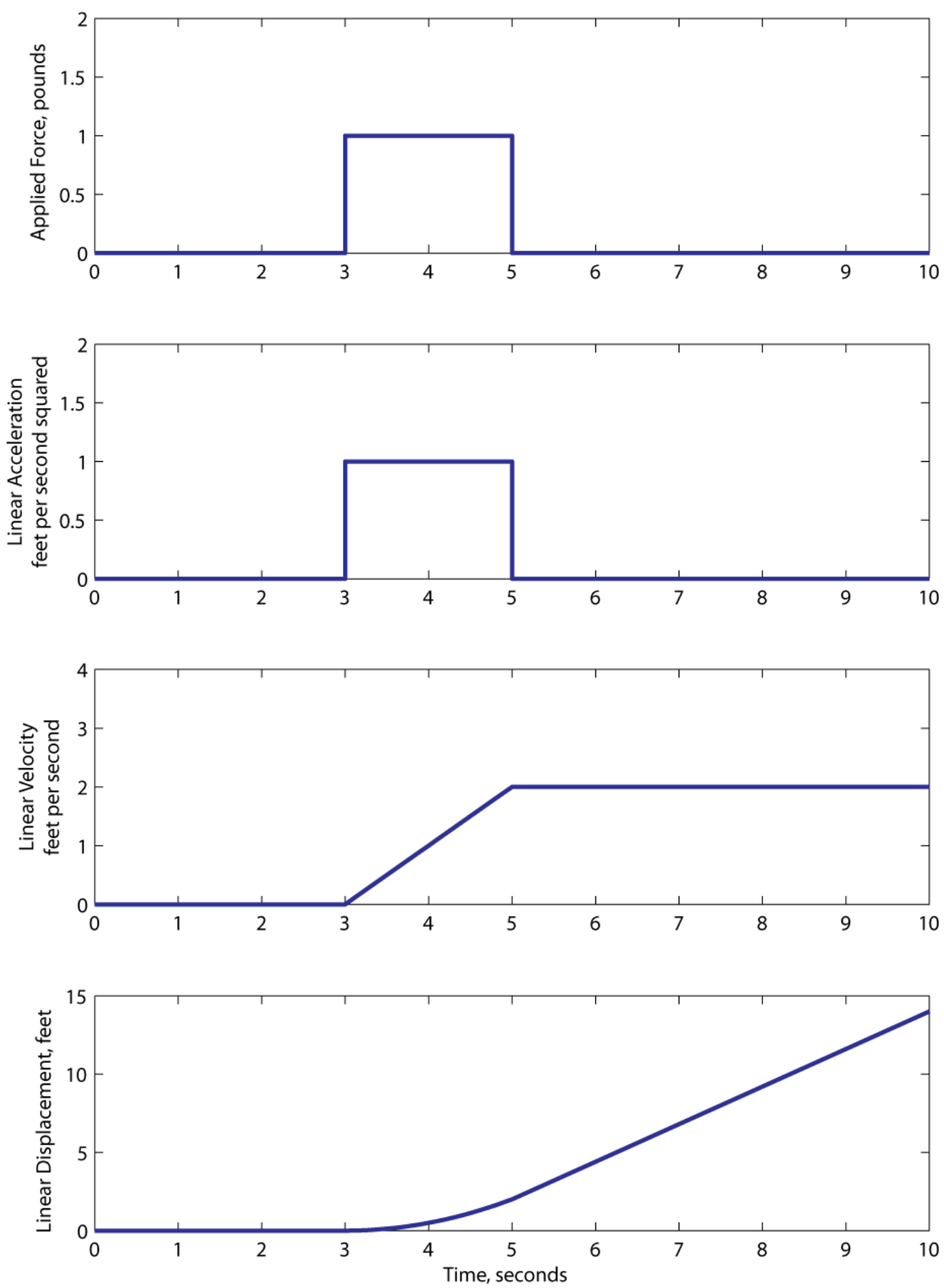
Angular validation for unit inertia, typical for all directions.
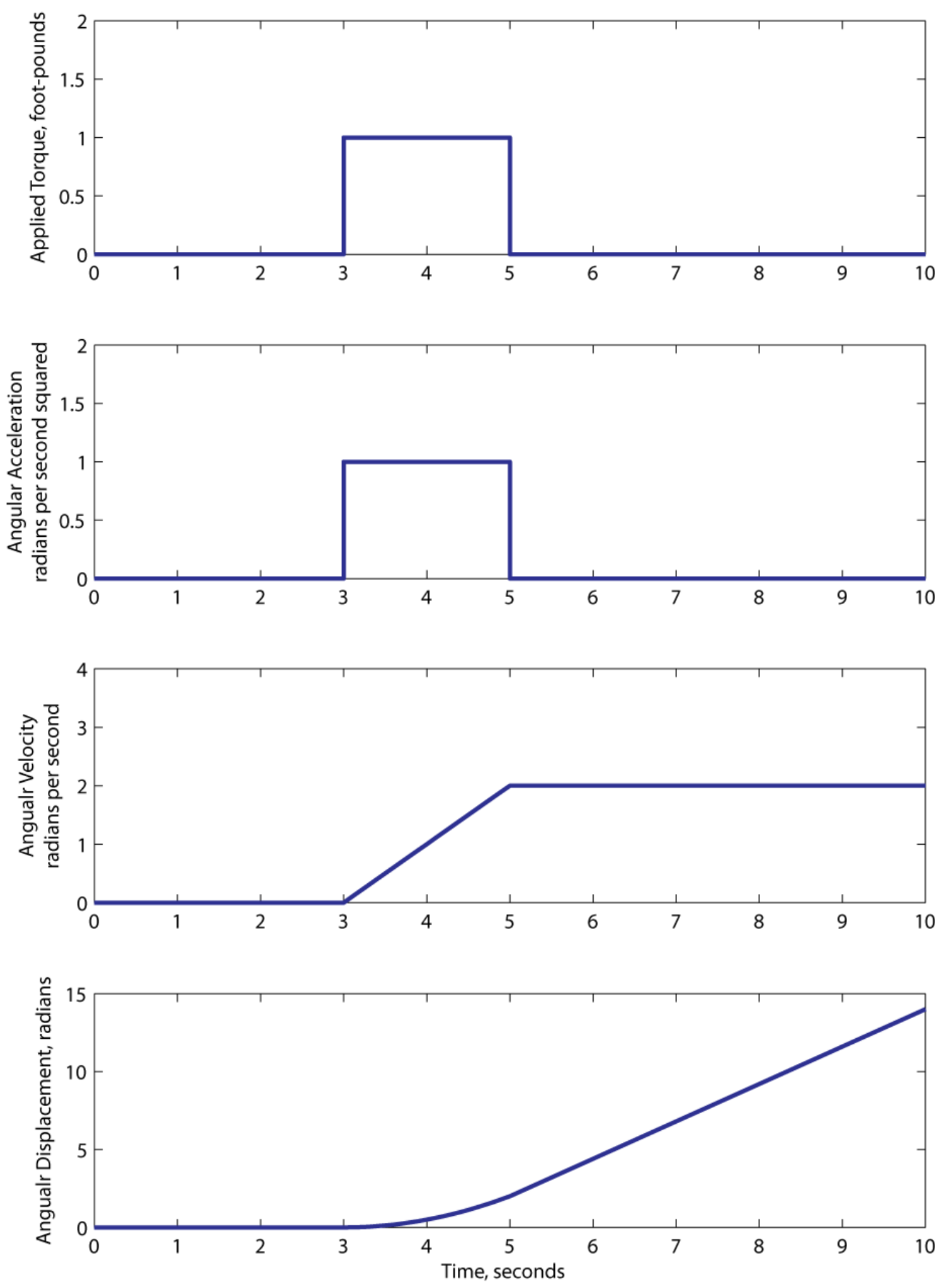


\title{
Appendix II - 14 CFR §25.1329
}

TITLE 14 - AERONAUTICS AND SPACE

\author{
CHAPTER I - FEDERAL AVIATION ADMINISTRATION, DEPARTMENT OF \\ TRANSPORTATION
}

SUBCHAPTER C - AIRCRAFT

PART 25 - AIRWORTHINESS STANDARDS: TRANSPORT CATEGORY AIRPLANES

subpart $\mathrm{f}$ - EQUIPMENT

25.1329 - Automatic pilot system.

(a) Each automatic pilot system must be approved and must be designed so that the automatic pilot can be quickly and positively disengaged by the pilots to prevent it from interfering with their control of the airplane.

(b) Unless there is automatic synchronization, each system must have a means to readily indicate to the pilot the alignment of the actuating device in relation to the control system it operates.

(c) Each manually operated control for the system must be readily accessible to the pilots.

(d) Quick release (emergency) controls must be on both control wheels, on the side of each wheel opposite the throttles.

(e) Attitude controls must operate in the plane and sense of motion specified in 25.777(b) and 25.779(a) for cockpit controls. The direction of motion must be plainly indicated on, or adjacent to, each control.

(f) The system must be designed and adjusted so that, within the range of adjustment available to the human pilot, it cannot produce hazardous loads on the airplane, or create hazardous deviations in the flight path, under any condition of flight appropriate to its use, either during normal operation or in the event of a malfunction, assuming that corrective action begins within a reasonable period of time.

(g) If the automatic pilot integrates signals from auxiliary controls or furnishes signals for operation of other equipment, there must be positive interlocks and sequencing of engagement to prevent improper operation. Protection against adverse interaction of integrated components, resulting from a malfunction, is also required.

(h) If the automatic pilot system can be coupled to airborne navigation equipment, means must be provided to indicate to the flight crew the current mode of operation. Selector switch position is not acceptable as a means of indication.

[Doc. No. 5066, 29 FR 18291, Dec. 24, 1964, as amended by Amdt. 2546, 43 FR 50598, Oct. 30, 1978] 


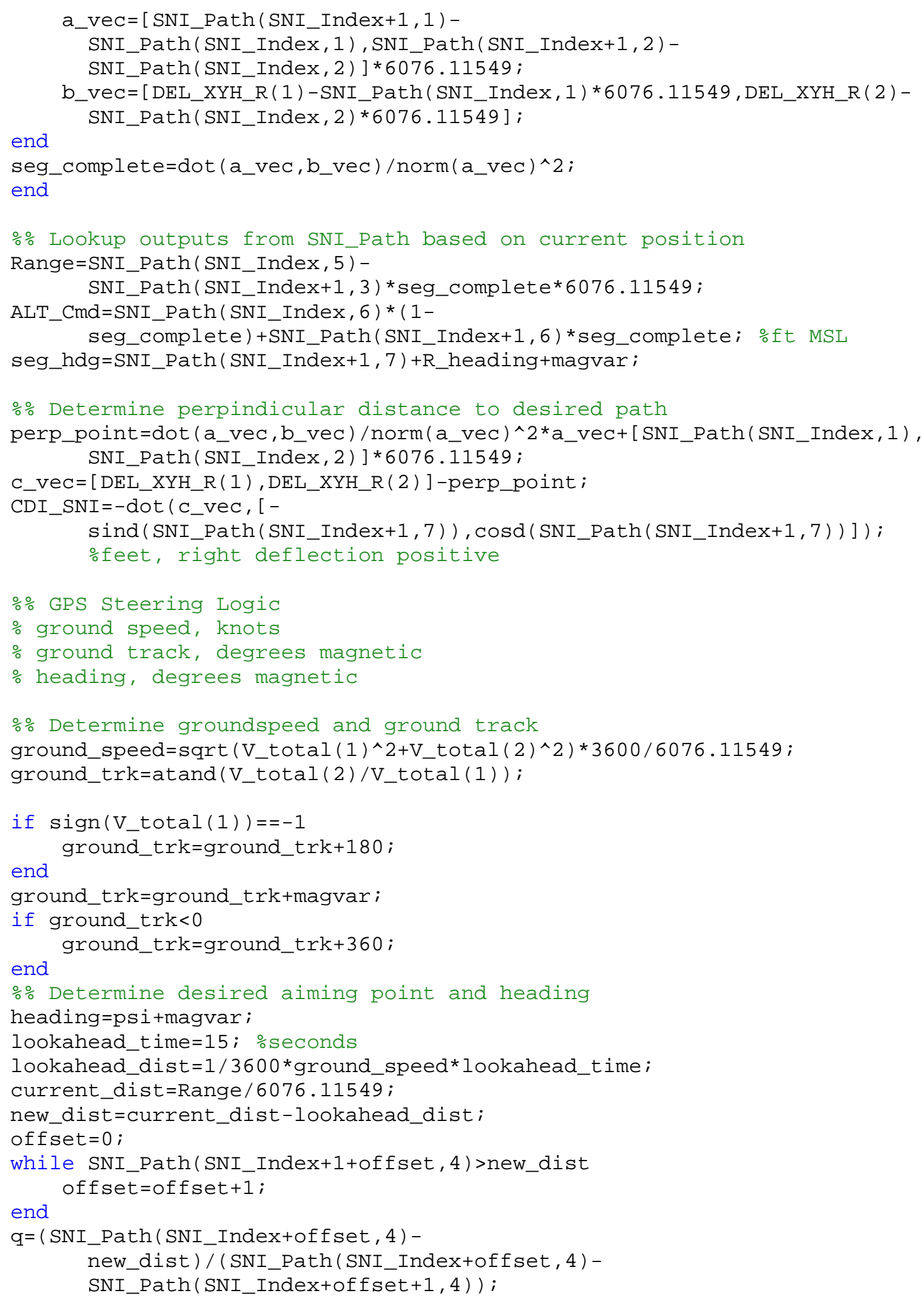




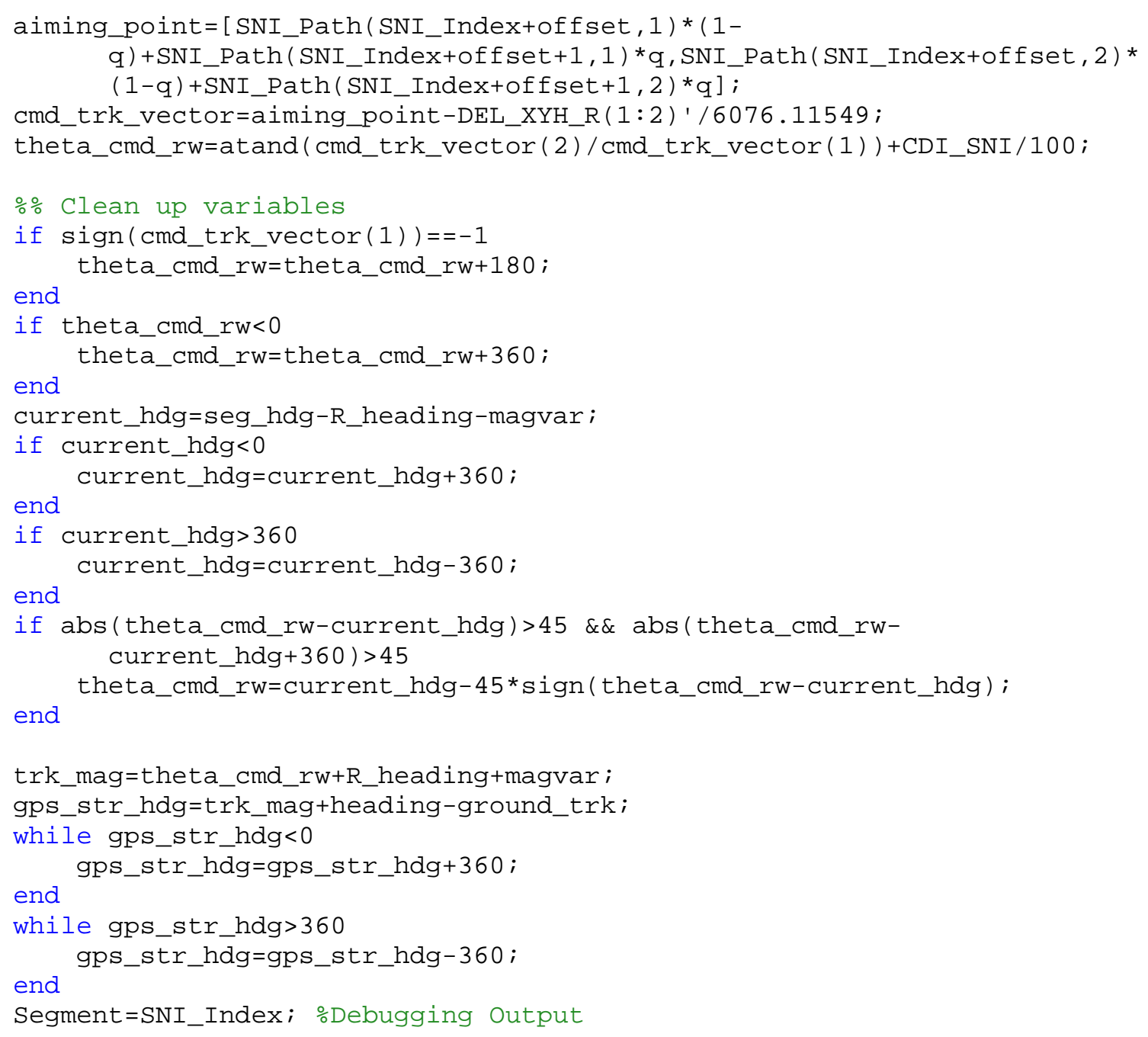

